สี

SSC-SR-1022

May 1986

Distribution Category: 414

SSC Central Design Group

\title{
An Assessment of the Antiproton-Proton Option for the SSC
}

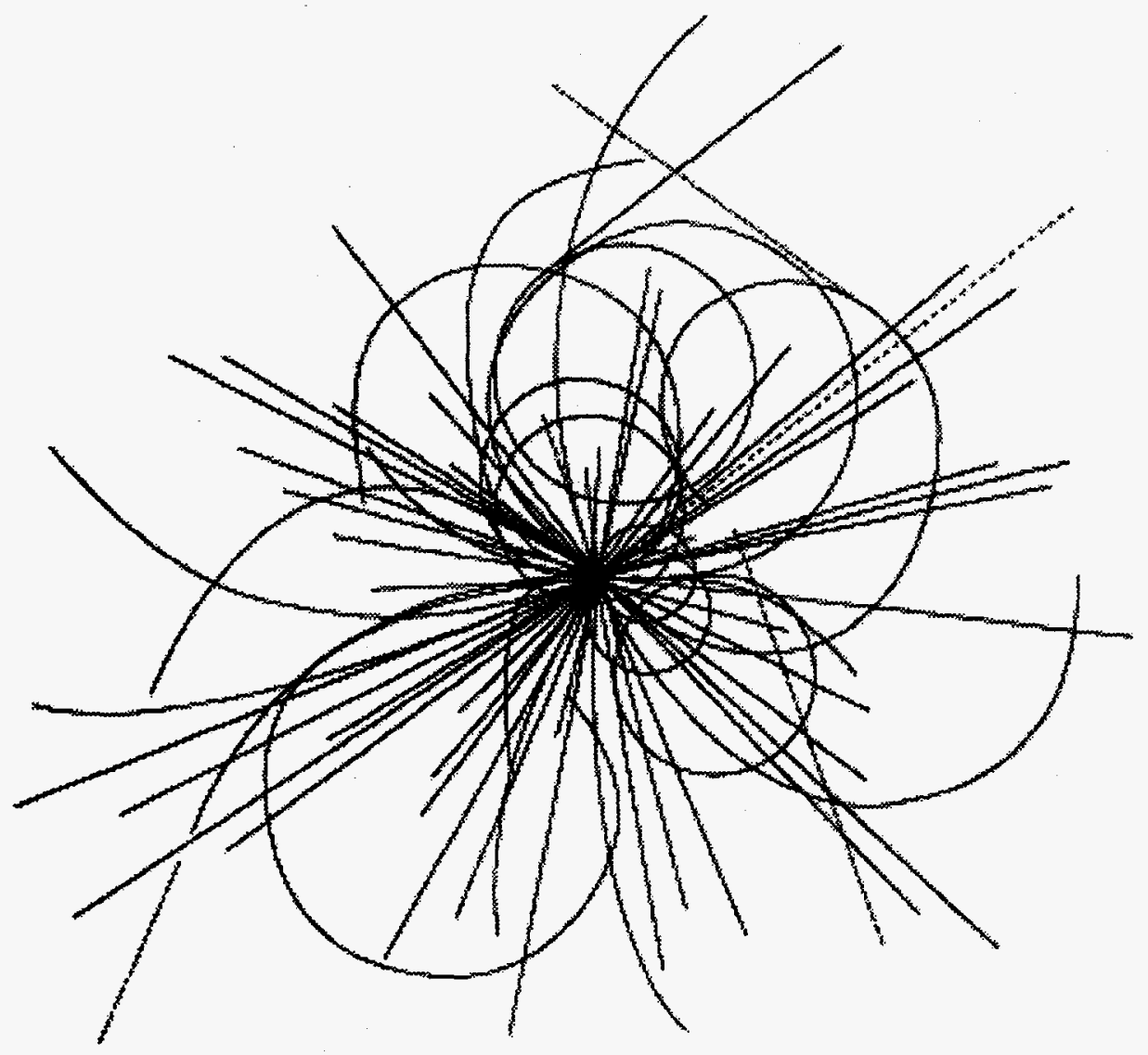

Superconducting Super Collider Laboratory

APPROVED FOR RELEASEOR

APPROVED FOR RELEASETENT GROUP

PUBLYCATION - OR. PATY $320 \%$..2.

\section{MASTER}




\title{
AN ASSESSMENT OF THE ANTIPROTON-PROTON OPTION \\ FOR THE SSC
}

\author{
$\overline{p p}$ Study Group \\ SSC Central Design Group* \\ c/o Lawrence Berkeley Laboratory \\ Berkeley, California 94720
}

May 1986

\section{DISCLAIMER}

This report was prepared as an account of work sponsored by an agency of the United States Government. Neither the United States Government nor any agency thereof, nor any of their employees, makes any warranty, express or implied, or assumes any legal liability or responsibility for the accuracy, completeness, or usefulness of any information, apparatus, product, or process disclosed, or represents that its use would not infringe privately owned rights. Reference herein to any specific commercial product, process, or service by trade name, trademark, manufacturer, or otherwise does not necessarily constitute or imply its endorsement, recommendation, or favoring by the United States Government or any agency thereof. The views and opinions of authors expressed herein do not necessarily state or reflect those of the United States Government or any agency thereof. 


\section{ANTIPROTON-PROTON STUDY GROUP}

B.C. Barish, Caltech (Chairman)

A. Chao, SSC/CDG

M. Harrison, SSC/CDG - Fermilab

R. Hollebeek, Univ. of Pennsylvania

D.E. Johnson, SSC/CDG - Fermilab

R. Kass, SSC/CDG - Ohio State University

A. Minten, CERN

S. Peggs, SSC/CDG

A. Wrulich, SSC/CDG, LBL 


\section{DISCLAIMER}

Portions of this document may be illegible in electronic image products. Images are produced from the best available original document. 


\title{
Foreword
}

A study to assess the antiproton-proton option for the SSC was initiated in February 1986 under the auspices of the SSC Central Design Group. We were asked by $M$. Tigner to coordinate this effort and to produce a report by May 1986. The goal was to understand what performance is technically feasible for

a $\overline{p p}$ collider, and how it compares in both physics potential and cost with the pp collider.

To perform this study, we formed a group at the CDG to investigate technical issues, assist with costing, etc. The members of this group are contained in the author list on the previous page. We particularly note the extensive work by $A$. Wrulich on separation schemes for the beams in the main collider ring and the requirements on the aperture of such a ring.

We received much advice and useful comments from many people. We note discussions with A.V. Tollestrup, J. Mariner, J. Peoples and S. Holmes (Fermilab), J. Simpson (Argonne), L. Evans, E. Jones, R. Billinge, J. Gareyte, G. Brianti and $W$. Kienzle (CERN). In addition, at CDG we appreciated the comments of $P$. Limon and $M$. Tigner.

Lastly we thank the staff at the CDG for their hospitality and help on the details of completing this study and writing the report.

\author{
B.C. Barish (Chairman) \\ R. Hollebeek \\ A. Minten
}




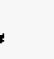




\section{CONTENTS}

Chapter

1

2

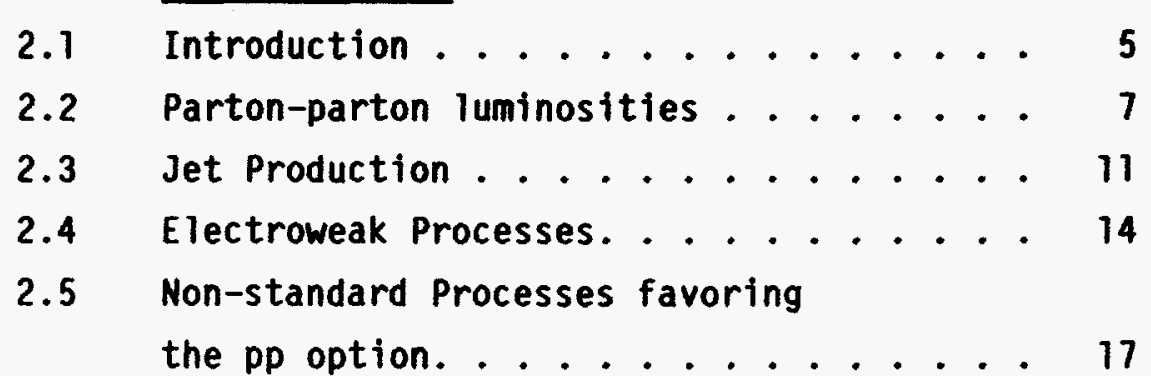

2.6 Non-standard Processes favoring

the $\overline{\mathrm{pp}}$ option. . . . . . . . . . 19

2.7 Summary. . . . . . . . . . . 22

3

3.1 Introduction ............ 25

3.2 Antiproton Sources........... 27

3.3 Scenario for the $\overline{p p}$ Supercollider. . . . 30

3.4 Operational Aspects. ......... 34

3.5 Advanced Schemes ........... 38

4

Machine Issues ............. . 41

4.1 Introduction ............. 41

4.2 Antiproton Source........... 42

4.3 Main Ring. . . . . . . . . . 51

\section{5}

Cost Implications. . . . . . . . . 109

5.1 Introduction ........... 109

5.2 Injector Systems ........... 110

5.3 Main Ring. . . . . . . . . . . 114

5.4 Conventional Facilities. . . . . . 117

5.5 Conclusions. . . . . . . . . . 118

Discussions and Conclusions. . . . . . 119 


\section{Chapter 1}

\section{INTRODUCTION}

The Conceptual Design Report $(C D R)^{7}$ for the Superconducting Super Collider (SSC) describes a proton-proton collider with an energy of $20 \mathrm{TeV}$ per beam and a maximum luminosity of $10^{33} \mathrm{~cm}^{-2} \mathrm{~s}^{-1}$ per collision point. This directly responds to the recommendation made by the High Energy Physics Advisory Pane 1 to the U.S. Department of Energy and the National Science Foundation in July 1983. That recommendation called for the "immediate initiation of a multi-TeV high-luminosity proton-proton collider project with the goal of physics experiments at this facility at the earliest possible date." The primary parameters of the SSC in the Conceptual Design Report have been chosen taking account of both the physics discovery reach of the machine and accelerator physics considerations.

The endeavor of the study reported here was to compare the feasibility of an antiproton-proton collider with the proton-proton collider presented in the SSC Conceptual Design Report. The rapid advances in the technology of $\overline{p p}$ colliders at CERN and Fermilab suggest that $\overline{p p}$ might be a viable alternative to a pp collider (or might be a first stage of an eventual pp collider). There is potentially a large cost saving from eliminating one $20 \mathrm{TeV}$ ring of magnets since the protons and antiprotons share the same ring. Following this suggestion, workshops at the University of Chicago $^{2}$ and at Snowmass $^{3}$ have provided a forum for these ideas. These reports formed the starting point for our study.

We have undertaken to determine how a $\overline{p p}$ collider would compare with a pp collider in luminosity, physics, cost, complexity, and reliability. In order to be as specific as possible, we have used the present and projected $\bar{p}$ sources at CERN and Fermilab as reference points, and have tried to make 
reasonable extrapolations in the technology to a $20 \mathrm{TeV}$ per beam $\overline{p p}$ collider. These extrapolations, though ambitious, do not involve some of the more speculative unproven schemes that have been suggested. We have "superposed" this extrapolated $\bar{p}$ source onto the pp design of the Conceptual Design Report and made the appropriate modifications to transform it into a $\overline{p p}$ collider. Although this is not an optimized $\overline{p p}$ solution, we believe it is good enough for us to understand the potential savings and trade-offs between $p p$ and $\overline{p p}$ colliders.

We have concentrated much of this study on a specific example of a $\overline{p p}$ collider of $20 \mathrm{TeV}$ per beam with luminosity $\mathscr{L} \simeq 10^{32} \mathrm{~cm}^{-2} \mathrm{sec}^{-1}$. This choice of parameters results from our analysis of what technical advances can reasonably be expected and of the various cost considerations. We discuss this $\overline{p p}$ collider in terms of its physics potential, the various technical issues, and the projected cost saving relative to the pp collider.

The main conclusions of this study are:

- a pp collider of $20 \mathrm{TeV}$ per beam at this luminosity appears to be technically feasible, with reasonable extrapolations in the technology:

- the difference between $p p$ and $\overline{p p}$ collisions for SSC physics is very sma 11, so the loss in luminosity for the $\overline{p p}$ option is the most important consideration;

- the cost saving of this $\bar{p} p$ collider is estimated to be approximately $\$ 242 M$ with respect to the pp collider of the CDR; 
- owing to its added technical complexity, the $\overline{p p}$ collider cannot be expected to achieve the same reliability and performance as the pp collider.

The following sections give a summar: of the general considerations for a $\overline{p p}$ collider, plus discussions of the physics potential, technical issues, cost, and a more detailed set of conclusions. 
References

1. Conceptual Design of the Superconducting Super Collider, SSC-SR-2020, SSC Central Design Group, March 1986.

2. $\overline{p p}$ Options for the Supercollider, Proceedings of a Workshop organized by Argonne National Laboratory and the University of Chicago, Chicago, February 1984.

3. Design and Utilization of the SSC, Proceedings of the 1984 Snowmass Summer Study, edited by R. Donaldson and J. G. Morf in, Snowmass, C0, 1984. 
Chapter 2

\section{PHYSICS ISSUES}

\subsection{Introduction}

The important parameters for determining the physics potential of a multiTeV collider are its energy and luminosity. For the purpose of this study, we fix the energy of both the $p$ and $\bar{p}$ options at $40 \mathrm{TeV}$, as in the COR, and the nominal luminosity of the pp option at $10^{33} \mathrm{~cm}^{-2} \mathrm{sec}^{-1}$. The $\overline{p p}$ option used for comparison has a luminosity of $-10^{32} \mathrm{~cm}^{-2} \mathrm{sec}^{-1}$. For some processes, there will be no difference in the expected cross-section from pp or pp initial states, so the major impact of using $\bar{p}^{\prime} s$ will be the loss of an order of magnitude in integrated luminosity. For other processes, $\bar{p}$ 's are more effective in producing the desired final state, and this difference can partially offset the lost luminosity.

\subsubsection{Parton Model}

A proton can be considered as a collection of quarks, gluons, and quarkantiquark pairs, each of which carries a fraction $x_{i} p$ of the longitudinal momentum $p$ of the proton. Transverse momenta carried by the constituents (or partons) are negligible at high energies.

The interaction cross-section of two protons to form a final state $x$ can be expressed as

$$
d \sigma(p p \rightarrow x)=\sum_{\substack{\text { partons } \\ i, j}} f_{i}^{(p)} f_{j}^{(p)} d \hat{\sigma}\left(i j \rightarrow x^{\prime}\right)
$$

The sum is over the probabilities of finding a parton of type $i$ or $j$ in the proton $\left(f_{i}^{(p)}\right)$ multiplied by the cross-section for the elementary subprocess 
that leads to the state $x$. The cross-section for the elementary subprocess can be calculated from the details of the underlying theory. The parton fluxes, which are functions of the momentum fraction $x_{i}$, can be determined from experiments at our present energy scale. This simple picture is modified by strong interaction (QCD) corrections, which can be incorporated by introducing a $Q^{2}$ or scale dependence in the flux factors. Using data from deep inelastic scattering to determine the parton distributions at $Q_{0}^{2}$, these functions can be evolved to other energies using the Altarelli-Paris ${ }^{1}$ equations. This procedure has been tested with data taken at the CERN SpDS Collider; the predicted distributions agree reasonably well with those found experimentaliy.

The evolution procedure is not exact and contains several uncertainties. In the small $\times$ region, present data do not constrain the value of the structure functions $f_{j}\left(x, Q^{2}\right)$, and the region below $x=0.01$ is parameterized by extrapolations of the behavior at slightly higher $x$ values. As the structure functions are evolved to higher values of $Q^{2}$, the differences due to different choices in the parameterization near $x=0$ tend to diminish, and since (as we shall see later) $\bar{p}$ and $p$ colliders are expected to differ in their parton fluxes only at high $x$ values, we can ignore this complication.

Additional uncertainties in the structure functions come from uncertainties in the QCD scale parameter $\Lambda$, from the use of nuclear targets rather than protons to determine the structure functions, and from the choice of data used for the low $Q^{2}$ parameterization. These problems lead to uncertainties of 10-20\% for moderate values of $x$. Although these uncertainties do affect the predicted rates for a given process, they tend to have the same effect on both the proton and the antiproton structure functions. 


\subsection{Parton-Parton Luminosities}

As discussed by Eichten et al (ref. 2, EHLQ), the luminosity $\mathscr{L}$ of a particular combination of initial partons colliding with c.m. energy $\sqrt{5}$, as illustrated in Fig. 2.1, is given by

$$
\tau \frac{d \mathscr{L}}{d \tau}=\frac{\tau}{1+\delta_{i j}} \int_{\tau}^{1}\left[f_{i}^{(p)}(x) f_{j}^{(p \pm)}(\tau / x)+f_{j}^{(p)}(x) f_{j}(p \pm)_{\tau / x)}\right] \frac{d x}{x},
$$

where

$$
\tau=x_{i} x_{j}=\hat{s} / s .
$$

This differential luminosity represents the number of parton-parton collisions of type $i j$ with $c . m$. energies in the range $\tau$ to $\tau+d \tau$. After multiplying by $1 / \mathrm{s}$, the typical form of a hard-scattering process, we obtain a quantity with the dimensions of a cross-section.

Since the primary difference between $p p$ collisions and $\overline{p p}$ collisions is the presence of valence antiquarks in the $\bar{p}$, it is interesting to compare the parton luminosity for valence-valence collisions in $\overline{p p}$ with valence-sea collisions in pp. For a process which requires quark anti-quark collisions, this is a measure of the degree to which antiprotons can compensate for lower machine luminosity. 


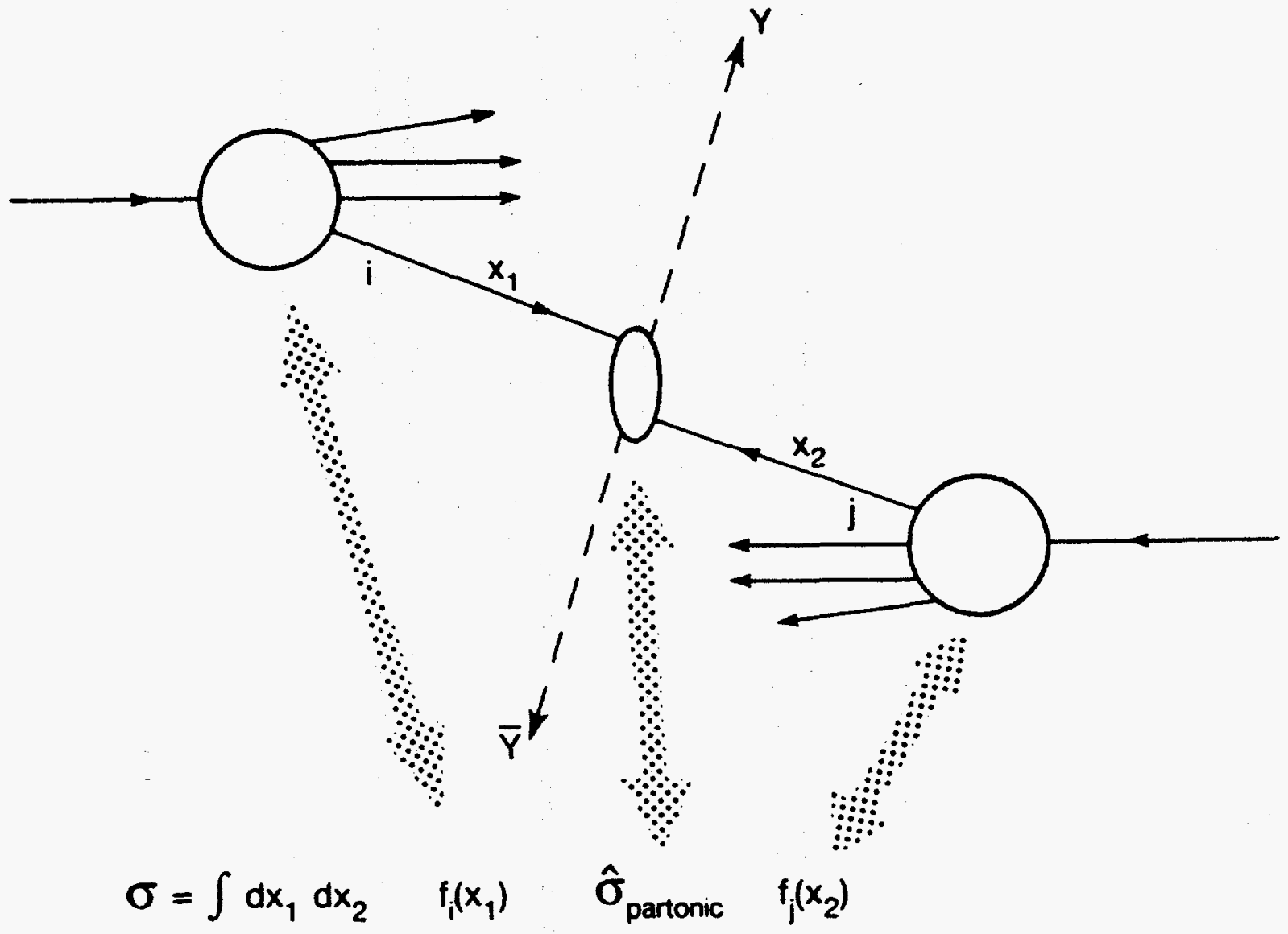

Figure 2.1. General parton-parton interaction. A parton of energy $x_{1} E$ in one proton collides with a parton of energy $x_{2} E$ from the other proton to produce a particle-antiparticle pair $Y \bar{Y}$ at large angles. The observed cross section is formed from the partonic cross section, folded with the respective probabilities of finding the specific partons of energies $x_{1} E$ and $x_{2} E$ within the two protons. 
Table 2.1 Ratio of $\tau / \mathrm{s}$ d $\mathscr{L} / \mathrm{d} \tau$ for $\overline{\mathrm{uu}}$ interactions in $\mathrm{pp}$ and $\overline{\mathrm{pp}}$
collisions at $\sqrt{\mathrm{s}}=40 \mathrm{TeV}$.

$\begin{array}{lc}\sqrt{\hat{s}}(T e V) & \overline{p p}(\bar{u} u) / p p(\bar{u} u) \\ 10^{-1} & 1 \\ 2 & 1.3 \\ 2.5 & 2.0 \\ 4.8 & 3.0 \\ 6.0 & 4.0 \\ 7.1 & 5.0 \\ 8.0 & 6.0 \\ 9.0 & 7.0 \\ 10 & 8.5\end{array}$

As can be seen from Table 2.1 , there will be a significant $\bar{p}$ advantage for processes above $4 \mathrm{TeV}$, or $\sqrt{\hat{s}} / \sqrt{s}>0.1$, but this advantage amounts to an order of magnitude in luminosity only for mass scales above $10 \mathrm{TeV}$. To take advantage of this difference, the hard scattering cross-section

$$
\sigma(\hat{s})=\frac{c}{\hat{s}}
$$

must be large enough that at the $10 \mathrm{TeV}$ mass scale, and for integrated luminosities of order $10^{39} \mathrm{~cm}^{-2}$, a significant number of events can be found. This will depend primarily on the size of the coefficient $c$, and the number of events necessary to separate the process from the background. As an example, if the coefficient $c$ is of order $\left(\alpha_{s} / \pi\right)^{2}$, typical of strong interactions, then at $10 \mathrm{TeV}$ we expect a cross-section of order $6 \times 10^{-39} \mathrm{~cm}^{-2}$, and hence we need integrated luminosities of at least $10^{39} \mathrm{~cm}^{-2}$ or more. This example illustrates that for processes with electroweak production rates, i.e. $(\alpha / x)^{2}$, it is unlikely that one would be able to take advantage of the increased parton-antiparton luminosity of a pp collider. The expected crosssections for several other subprocesses are shown in Table 2.2 for $p p$ and $\overline{p p}$ 
collisions with subprocess c.m. energies of 1,3 and $10 \mathrm{TeV}$. Note that the

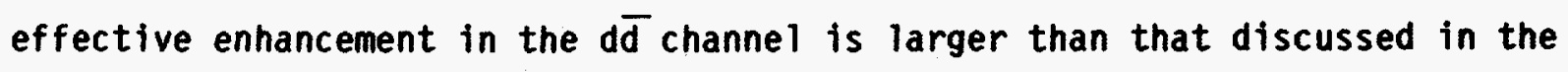
uu channel because the parameterization ${ }^{3}$

$$
d_{v}(x) / u_{v}(x)=0.57(1-x)
$$

requires a higher average $x$ value for the antiquark for a fixed $\hat{s}$.

Table 2.2

$\tau / \widehat{s} \frac{\mathrm{d} \mathscr{L}}{\mathrm{d} \tau}(\mathrm{nb})$ for parton-parton

interactions with $\sqrt{\hat{s}}=1,3$ and $10 \mathrm{TeV}$

$\sqrt{\hat{s}}=$

1

3

10

\begin{tabular}{lllllll}
\hline Subprocess & $p p$ & $\overline{p p}$ & $p p$ & $\overline{p p}$ & $p p$ & $\overline{p p}$ \\
\hline uU & 1.0 & 1.0 & $2 \times 10^{-2}$ & $4 \times 10^{-2}$ & $4 \times 10^{-5}$ & $4 \times 10^{-4}$ \\
uu & 1.0 & 0.4 & $4 \times 10^{-2}$ & $10^{-2}$ & $4 \times 10^{-4}$ & $2 \times 10^{-5}$ \\
$\bar{u} \bar{u}$ & 0.13 & 0.4 & $1.5 \times 10^{-3}$ & $10^{-2}$ & $8 \times 10^{-7}$ & $2 \times 10^{-5}$ \\
dd & 0.7 & 1.3 & $10^{-2}$ & $5 \times 10^{-2}$ & $10^{-5}$ & $6 \times 10^{-4}$ \\
dd & 0.5 & 0.3 & $1.2 \times 10^{-2}$ & $5 \times 10^{-3}$ & $5 \times 10^{-5}$ & $7 \times 10^{-6}$ \\
ud & 1.3 & 0.5 & $4 \times 10^{-2}$ & $10^{-2}$ & $2 \times 10^{-4}$ & $2 \times 10^{-5}$
\end{tabular}




\subsection{Jet Production}

In the QCD parton model, jets of hadrons will be produced following a scattering process when the partons materialize into hadrons. The details of this hadronization process reflect non-perturbative effects, but perturbative methods nonetheless indicate that jets should become narrower and more easily distinguished from background processes as the jet energy increases. This trend has already been seen both for $p p$ and $\overline{p p} c 011$ isions ${ }^{4-6}$ and for $\mathrm{e}^{+} \mathrm{e}^{-}$ collisions. 7,8 Deviations from the expected QCD behavior of single jets would signal the onset of new physics, and might be evidence for quark compositeness. Dijet mass spectra can be used to search for new particles, for example new electroweak bosons, or Higgs particles with two jet decays. Within QCD, one expects to see at high enough energies a difference in the multiplicity and angular spread of gluon jets. If this is realized on the scale of SSC jets, it will be an extremely useful tool for understanding jet phenomena.

Single jet production receives contributions from many parton subprocesses, such as

$$
q q^{\prime} \rightarrow q q^{\prime}, q \bar{q} \rightarrow q q^{\prime} \bar{q}^{\prime}, q \bar{q} \rightarrow g g, g g \rightarrow q \bar{q}, g q \rightarrow g q, g g \rightarrow g g .
$$

At small transverse momenta $(<1 \mathrm{TeV})$, the cross-section is dominated by processes which yield two gluon final states through either $\mathrm{gg} \rightarrow \mathrm{gg}$ or $\mathbf{q q} \rightarrow \mathbf{g g}$. At higher values $(<4 \mathrm{TeV})$, the dominant process is qg scattering. Since these processes do not involve valence-valence scattering, the cross-section is the same for $p p$ and $\overline{p p}$ collisions. To estimate the effect of a loss in luminosity of a factor of 10 , we assume an integrated luminosity of

$$
\operatorname{Te}_{T_{\mathscr{L}}} \mathscr{L}
$$


where

$$
\begin{aligned}
T & =3 \times 10^{7} \frac{\mathrm{sec}}{\text { year }} \times 2 \text { years } \\
\varepsilon_{T} & =0.5=\text { fraction of year spent in running } \\
\varepsilon_{\mathscr{L}} & =\frac{1}{3}=\frac{\mathscr{L}(\text { average })}{\mathscr{L}(\text { peak })}
\end{aligned}
$$

Which yields $10^{39} \mathrm{~cm}^{-2}$ integrated luminosity from a peak luminosity of $10^{32} \mathrm{~cm}^{-2} \mathrm{sec}^{-1}$. This is probably an optimistic estimate of what would be accumulated in a 2 year $\overline{p p}$ run, since the ratio of the average to the peak luminosity may be less than assumed, depending on the reliability and availability of the $\bar{p}$ source. We assume that the integrated luminosity for the pp option would be $10^{40} \mathrm{~cm}^{-2}$.

The single jet energy resolution in the 4-6 TeV range is not likely to be better than 1\%, so we require a sensitivity of 10 events in a $50 \mathrm{GeV}$ bin and a single unit of rapidity. This corresponds to a cross-section sensitivity of $2 \times 10^{-7} \mathrm{nb} / \mathrm{GeV} / \mathrm{c}$, which for $\sqrt{\mathrm{s}}=40 \mathrm{TeV}$ occurs at $\mathrm{p}_{\mathrm{T}}=4.2 \mathrm{TeV} / \mathrm{c}$. The single jet cross-section in this region is falling like

$$
\left.\frac{d o}{d p_{T} d y}\right|_{y=0} \sim p_{T}^{-7.3}
$$

so the pp machine with integrated luminosities of $10^{40} \mathrm{~cm}^{-2}$ would probe $\mathrm{p}_{\mathrm{T}}$ values to $5.8 \mathrm{TeV} / \mathrm{c}$. Detailed studies of the $\mathrm{p}_{\mathrm{T}}$ or $\mathrm{y}$ behavior of the jets would require more events, and hence smaller $p_{T}$ scales, favoring the choice of a pp machine.

Dijet production is also dominated by gq subprocesses for dijet masses less than $9 \mathrm{TeV}$, so again one does not expect a difference in cross-section between $\mathrm{pp}$ and $\overline{\mathrm{pp}}$. For an integrated luminosity of $10^{39} \mathrm{~cm}^{-2}$ and a sensitivity of 10 events $/ 35 \mathrm{GeV}$ bin, the $\overline{p p}$ option can reach jet pair masses of 
$9 \mathrm{TeV} / \mathrm{c}^{2}$, with both jets constrained to lie in the rapidity interval $|y|<1.5$. Since the cross section in this region varies like

$$
\frac{d \sigma}{d M} \sim M^{-7} \text {, }
$$

a factor of 10 effective increase in luminosity for the pp option would probe to $12.5 \mathrm{TeV} / \mathrm{c}^{2}$.

The conclusions for multijet production are similar to those for dijet production. Since the integrated luminosities are not large enough to reach the region where valence-valence effects are important, the pp and $\overline{p p}$ crosssections are the same. Three-jet production can be probed to a sensitivity of $3 \times 10^{-7} \mathrm{nb} / \mathrm{GeV}$ or $7.2 \mathrm{TeV}$ in the three jet invariant mass with the $\overline{\mathrm{p}}$ option. Since the cross-section in this mass range varies as

$$
\frac{d a}{d x_{1} d x_{2} d y_{\text {boost }} d \cos \theta} \sim M^{-7}
$$

the pp sensitivity would be $10 \mathrm{TeV} / \mathrm{c}^{2}$. The dominant subprocesses are ggg and gga production.

Table 2.3

Comparison of $p p$ and $\overline{p p}$ Sensitivities For
Jet Production.

$\begin{array}{lll}\text { Process } & \mathrm{pp} & \overline{\mathrm{pp}} \\ \text { Single jet } \mathrm{p} & 5.8 & 4.2 \\ \text { Dijet mass } & 12.5 & 9.0 \\ \text { Trijet mass } & 10.0 & 7.2\end{array}$




\subsection{Electroweak Processes}

\subsubsection{Dilepton production}

Dilepton production in hadron collisions has traditionally been a powerful means of searching for new physics. For an SSC scale machine, the reaction

$$
\mathrm{pp} \text { or } \overline{\mathrm{pp}} \rightarrow \ell^{+} \ell^{-}+\text {anything }
$$

will proceed in lowest order through quark-antiquark annihilation, for example

$$
\mathrm{q} \overline{\mathrm{q}} \rightarrow r^{\star} \rightarrow \ell^{+} \ell^{-} \text {or } \mathrm{q} \overline{\mathrm{q}} \rightarrow \mathrm{z}^{0} \rightarrow \ell^{+} \ell^{-}
$$

For a pp machine with integrated luminosity of $10^{40} \mathrm{~cm}^{-2}$, the yield is one event per $\mathrm{GeV} / \mathrm{C}^{2}$ per day for $\mathrm{M}^{+} l^{-}=700 \mathrm{GeV} / \mathrm{C}^{2}$. The reason the mass scale is lower than those discussed for jet production is that the coupling strength in this process is electroweak rather than strong. Because of the small mass scale relative to $\sqrt{\mathrm{s}}=40 \mathrm{TeV}$, there is no advantage in using $\overline{\mathrm{p}}^{\prime} \mathrm{s}$.

As an example, the Snowmass study of muon detection ${ }^{9}$ quotes a muon resolution of $5 \% \Delta \mathrm{p} / \mathrm{p}$ at $300 \mathrm{GeV}$. Requiring 10 events in a $25 \mathrm{GeV}$ bin gives a sensitivity up to mass scales of order $500 \mathrm{GeV}$ for $\overline{\mathrm{pp}}$, and $1 \mathrm{TeV}$ for $\mathrm{pp}$ with 10 times the integrated luminosity. The higher mass scales $(\sim 10 \mathrm{TeV})$, where there would be a $\bar{p}$ advantage, are inaccessible for this process because of the small coupling strength and small integrated luminosity.

\subsection{2 $W$ and $Z$ production}

Singly produced $W^{\prime}$ 's and $Z^{\prime}$ 's are being studied at the present generation of colliders. At the SSC, they may still be of use for detector calibration and, since the production rates are large, for the study of rare decays.

The total cross-section for $W^{ \pm}$production in fo collisions where the $W$ is restricted to lie in the rapidity interval $|y|<1.5$ is $35 \mathrm{nb}$, yielding an event sample of $3.5 \times 10^{8}$. events. The production proceeds through the 
reaction $\overrightarrow{q q} \rightarrow W+g$ and $g+q \rightarrow W+q$, and because of the small mass scale $\left(M_{w}\right)$ the total cross-section for $\overline{p p}$ is similar $(33 \mathrm{nb})$. The collected event sample would therefore be 10 times smaller. Note however that the total production rates of $\mathrm{W}^{-}$and $\mathrm{W}^{+}$are equal in $\overline{\mathrm{p} p}$, whereas in $\mathrm{pp}$ the smaller momentum fraction carried by down quarks tends to suppress the production of $W^{-}$relative to $W^{+}$. The total rate for $W$ production is approximately three times larger than the rates with $|y|<1.5$.

Pairs of gauge bosons are produced primarily through $q \bar{q}$ annihilation. Table 2.4 shows the effect of a factor of ten loss in luminosity on the maximum accessible pair mass; both bosons are restricted to the range $|y| \leq 2.5$. Much of the cross-section is mediated by the annihilation of sea quarks with sea antiquarks.

Table 2.4

Maximum Accessible Pair Mass (TeV) for Gauge Bosons Produced in $|y| \leq 2.5$ with, $10^{40}\left(10^{39}\right) \mathrm{cm}^{-2}$ luminosity $\mathrm{pp}(\overline{\mathrm{pp}})$ Collisions.

$\begin{array}{lll}\text { Process } & \text { pp } & \overline{p p} \\ W^{+} W^{-} & 2 & 1.3 \\ W Z & 1.7 & 1.1 \\ Z Z & 1.4 & 0.8\end{array}$

\subsubsection{Higgs Boson Production}

This process is probably one of the most important to be investigated by the SSC if the Higgs mass is greater than a few hundred GeV. The direct annihilation of quark and antiquarks has a small cross section, and the dominant production mechanisms are gluon-gluon fusion through a quark $100 p^{10}$ or intermediate boson fusion ${ }^{11}$, the exact ratio depeniing on the mass of the top quark. 
Since the production mechanisms are dominated by processes which do not require valence antiquarks, the $p p$ and $\overline{p p}$ cross-sections are again the same. The cleanest signal would probably be the detection of $Z$ pair final states. The ratio of Higgs branching ratios to $Z$ and $W$ pairs varies between $1 / 3$ and 1/2 depending on the Higgs mass. If we assume that approximately $30 \%$ of the $Z$ decays will be reconstructed, then the rates are sufficient to probe a Higgs mass of 1.3 and $0.85 \mathrm{TeV}$ for $\mathrm{pp}$ and $\overline{\mathrm{pp}}$ respectively. In practice, it may be difficult to establish unambiguously the existence of a Higgs which is heavier than $600 \mathrm{GeV}$, because the total width becomes greater than $100 \mathrm{GeV}$. A $1 \mathrm{TeV}$ Higgs may have a width of several hundred GeV.

In the $W$ pair channel, the rates are higher because of the larger decay branching ratio, but it will probably be more difficult to identify W's. Backgrounds come from the $Q C D$ production of $W$ pairs discussed previously, and are $\sim 20 \%$ higher for $|y|<2.5$ in $\bar{p}$ production because of the lack of suppression of $w^{-}$production. For the central region $|y|<1.5$, the backgrounds should be comparable, and the major impact is the loss in luminosity. 


\subsection{Non-standard processes favoring the pp option}

\subsubsection{Heavy Quarks and Heavy Leptons}

In both of these processes, $\bar{p} p$ production has an advantage over pp production because the mechanisms involve quark-antiquark annihilation. The conclusions, however, are similar to those which have been reached before. The gain is not significant in comparison to a loss of a factor of ten in integrated luminosity over the mass ranges where the rates are high enough to make heavy quarks and leptons detectable with integrated luminosities of order $10^{39}-10^{40} \mathrm{~cm}^{-2}$.

\subsubsection{Technicolor and Supersymmetry}

To the list of processes which benefit from luminosity more than from the presence of antiquarks, we should add most technicolor and supersymmetric reactions. For example, the production of a technirho in the $\mathrm{W}^{+} \mathrm{W}^{-}$channel has for $W$ pairs near the expected mass of the technirho in a minimal model ( $1.8 \mathrm{TeV})$, a $25 \%$ higher cross-section in the $\overline{\mathrm{pp}}$ versus the pp case, but this does not compensate for the loss in luminosity. Similar conclusions hold for charged technirhos in the WZ channel. Integrating the spectrum between $1.5<$ $M_{Z W}<2.1 \mathrm{TeV}$, one obtains 130 background events and 550 signal events from an integrated luminosity of $10^{40} \mathrm{~cm}^{-2}$. Since the charge of the technirho can probably be investigated only by using the leptonic decays of the $W$, an integrated luminosity of at least $10^{40} \mathrm{~cm}^{-2}$ will be required unless the $\rho_{T}$ is lighter than expected. Lower mass states such as the technipion, or color octet neutral technipions, are produced at mass scales and rates for which there is no $\bar{p}$ advantage. 
In supersymmetry, production of gluino pairs is dominated by the reaction $g g \rightarrow \widetilde{g} \widetilde{g}$. Similarly, production of $\widetilde{\gamma} \widetilde{q}, \widetilde{z} \widetilde{q}$ and $\widetilde{w} \widetilde{q}$ is in each case dominated by gq scattering, leading to equal $p p$ and $\overline{p p}$ cross-sections. Table 2.5 shows the sensitivities to gaugino masses, if all such masses are equal, from events of the type pp or $\overline{p p} \rightarrow \widetilde{g} \widetilde{\gamma}, \widetilde{Z} \tilde{\gamma}$. Production of final states of the type $\widetilde{\gamma}$. $\widetilde{Z Z}$ or $\tilde{\ell} \tilde{l}^{*}$ occur with cross-sections which are too small for the $\overline{p p}$ option to be favored.

Table 2.5

Events required

to establish a

Process

signa 1

$p p$ or $\overline{p p} \rightarrow \widetilde{\mathrm{gz}}$

$p p$ or $\bar{p} p \rightarrow \tilde{g} \tilde{z}$

$p p$ or $\overline{p p} \rightarrow \tilde{g r}$ (stable)

$p p$ or $\overline{p p} \rightarrow \tilde{g r}$ (stable)
100

10

100

20

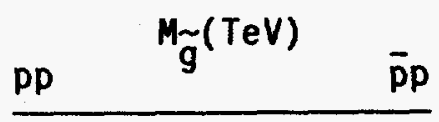

1.1

0.6

2.0

1.3

1.2

0.7

1.9

1.1 


\subsection{Non-standard Processes Favoring the $\overline{p p}$ Option}

We have found that in order to take advantage of the $\bar{p} p$ option, we must have both a coupling strength which is large enough to make valence interactions accessible, and a process which requires anti-quarks. Two areas where these conditions are met, and in which the $\bar{p}$ advantage is significant enough to compensate for a factor of ten loss in luminosity, are the production of new gauge bosons and the investigation of composite models of quarks and leptons. While both these processes are outside our current models, and hence rather speculative, they are nevertheless important extensions to our present models.

\subsubsection{New Gauge Bosons}

Many extensions to the standard electroweak model have been suggested, and most imply the existence of new gauge couplings and gauge bosons. If the couplings are comparable in size to those with which we are familiar in $S U(2)_{L}$, then the decays will be similar for $W^{\prime}$ and $W$ and $Z^{\prime}$ and $Z$. The production cross-sections will be smaller by the ratio

$$
\left[\frac{M_{w}}{M_{w^{\prime}}}\right]^{2} \text {. }
$$

Since the scaling variable becomes $M_{W^{\prime}}^{2} / s$ instead of $m_{W}^{2} / s$, valence contributions are more important. Remember that $u \bar{u}$ and $d \bar{d}$ luminosities are 10-60 times greater for $\overline{p p}$ than for $p p$ at $\sqrt{\tau} \sim 0.25$.

The discovery criteria used in EHLQ for this process is 1000 events produced in $\left|y_{w}\right|<1.5$, which would provide a convincing signal in either the $e$, the $\mu$, or the $\tau$ channel if the decay widths are

$$
\frac{\Gamma\left(W^{\prime} \rightarrow \ell_{v}\right)}{\Gamma\left(W^{\prime} \rightarrow \mathrm{a} 11\right)}=\frac{1}{4 N_{g}}
$$


and

$$
\frac{\Gamma\left(Z^{\prime} \rightarrow \ell^{+} l^{-}\right)}{\Gamma\left(Z^{\prime} \rightarrow \mathrm{a} 11\right)} \approx \frac{0.09}{N_{g}}
$$

where $\mathrm{N}_{\mathrm{g}}$ is the number of generations. This requirement is probably too strict, since the signals for all three and perhaps four types of leptons may be usable as well as some of the hadronic modes. The maximum boson mass for a sensitivity of 100 events produced is shown in Table 2.6 for $W^{\prime}$ and $Z^{\prime}$ production.

Table 2.6

\begin{tabular}{ccc} 
Process & \multicolumn{1}{c}{$M_{W^{\prime}, Z^{\prime}(\text { TeV })}$} & $\overline{\mathrm{pp}}$ \\
$\mathrm{pp}$ or $\overline{\mathrm{pp}} \rightarrow W^{\prime+}$ & 9.0 & 7.0 \\
pp or $\overline{\mathrm{pp}} \rightarrow W^{\prime-}$ & 7.5 & 7.0 \\
pp or $\overline{\mathrm{pp}} \rightarrow \mathrm{Z}^{\prime 0}$ & 8.2 & 11.0
\end{tabular}

The $\overline{p p}$ option in this case is approximately equivalent for $W^{\prime}$ production, and is favored for $\mathrm{Z}^{\circ}$ production. Should the coupling of the new gauge bosons for some reason be stronger than in the standard mode 1 the $\overline{p p}$ option would be more strongly favored.

\subsubsection{Composite Quarks and Leptons.}

From the observation that quarks and leptons can be grouped in successively heavier families or generations, it is natural to suspect that there may be a deeper level of substructure. If quarks and leptons are indeed composite objects, we may be able to construct all of the known objects from a smaller set of building blocks, often called preons. Theze particles would interact with a new strong force. If such an interaction exists above some scale $\Lambda$, then for parton subenergies $\sqrt{\hat{s}}>\Lambda$, the cross-section for parton-parton 
interactions should rise from $\pi \alpha_{s}^{2} / s$ to of order $4 \pi / \Lambda^{2}$. Two processes which would provide sensitive tests are production of high transverse momentum jets and of dileptons. The new subprocesses which would enhance these cross sections are

$$
\begin{aligned}
& q \bar{q} \rightarrow q \bar{q} q^{\prime} \bar{q}^{\prime}, \\
& q \bar{q} \rightarrow q \bar{q} \ell^{+} \ell^{-}, \\
& q \bar{q} \rightarrow \ell^{+} \ell^{-} \ell^{+} l^{-} .
\end{aligned}
$$

and

EHLQ estimate that by requiring at least a factor of two change in the cross section and at least 50 events total, a pp machine can detect $\Lambda$ values somewhere between 15 and $20 \mathrm{TeV}$. The $\overline{p p}$ option with one tenth the luminosity can reach approximately $13 \mathrm{TeV}$.

In the dilepton case, however, the standard model cross section for $\ell^{+} \ell^{-}$production is smaller than that for dijet production by a factor $\left(\alpha / \alpha_{s}\right)^{2}$, so the new contact interaction introduces a larger change in the observed cross-section. One might also argue that the signature for dileptons is cleaner than that for high $\mathrm{p}_{\mathrm{T}}$ jets. In this case, requiring again a factor of two change in rate, but also at least 75 events within $|y|<1.5$ gives a sensitivity of about $28 \mathrm{TeV}$ for the pp option with $10^{40} \mathrm{~cm}^{-2}$ integrated luminosity, and $\sim 25 \mathrm{TeV}$ for the $\overline{\mathrm{pp}}$ option with $10^{39} \mathrm{~cm}^{-2}$ integrated luminosity. If fewer events are required to establish the signal, because for example several generations of leptons are used, the $\overline{p p}$ option is favored. 


\subsection{Summary}

For most processes which have been considered, either there is no advantage in having valence antiquarks because the process receives equal qq and $q \bar{q}$ contributions (strong interactions) or the $\bar{q} q$ interaction coupling strength is of order $\alpha$. Such a small coupling strength restricts the mass scales of accessible processes to small values of $\tau$ because of the limited integrated luminosity $\left(10^{39}-10^{40} \mathrm{~cm}^{-2}\right)$. In most of these cases, the loss in physics reach of the $\overline{p p}$ collider is directly related to its smaller peak luminosity. Whenever the signal being investigated is spectacular and the number of events required is small, the $\overline{p p}$ advantage is increased. In analyzing the various standard model processes, we have tended to be optimistic about the number of events required for detection.

The two cases where the $\overline{p p}$ option is equal to or slightly favored over the pp option are both cases where a new interaction is postulated which couples strongly to the $\bar{q} q$ state. Other models of this type may exist or be suggested in the future, but the requirement that the coupling strength or cross section be larger than the scale of the electroweak point interactions is quite general. It results from the mass scale achievable with integrated luminosities of $10^{39}-10^{40} \mathrm{~cm}^{-2}$. A comparison of machines with $\overline{p p}(\mathrm{pD})$ luminosities of $10^{40}\left(10^{41}\right) \mathrm{cm}^{-2}$ would favor the $\overline{\mathrm{pp}}$ option more than the present case. 
References

1. G. Altarelli and G. Parisi, Nucl. Phys. B126, 298 (1977).

2. E. Eichten, I.J. Hinchliffe, K.D. Lane, C. Quigg, Rev. Mod. Phys. 56, 579 (1984).

3. F. Eisele, in Proceedings of the 21 st International Conference on High Energy Physics, edited by P. Petrace and M. Pornefu, J. Phys. (Paris) 43, Supp 1. 12, C3-337 (1982).

4. G. Arnison et. al., Hadronic Jet Production at the CERN Proton-Antiproton Collider, CERN-EP/83-118 (1983).

5. P. Bagnaia et a1., Z. Phys. 620, 117 (1983).

6. M. G. Albrow, in Proceedings of the 1983 International Europhysics Conference on High Energy Physics, edited by J. Guy and C. Costain (Rutherford Laboratory, Chilton, England), p 134.

7. G. Hanson et a 1., Phys. Rev. Lett. $\underline{35}, 1609$ (1975).

8. K. H. Mess and B.H. Wiik, in Gauge Theories and High Energy Physics, 1981 Les Houches lectures, edited by M.K. Gaillard and R. Sorta (North-Holland, Amsterdam, 1985), p. 865 .

9. R.M. Barnett and H.E. Haber, DPF Summer Study on the Design and Utilization of the SSC, June 23-July 11, 1984, Snowmass, Colorado, edited by R. Donaldson and J.G. Morfin, P. 296.

10. H. Georgi et a1., Phys. Rev. Lett, 40,692 (1978).

11. S. Petcov and D.R.T. Jones, Phys. Lett. 84B, 440 (1979). 
צ

$-$ 
Chapter 3.

\section{OVERVIEW OF THE $\overline{D P}$ OPTION}

\subsection{Introduction}

The aim of this chapter is to outline a possible alternative to the proposed SSC design, which has a pair of storage rings guiding two proton beams in opposite directions; the two rings intercept at six locations where the

protons collide and interact. ' The alternative consists of a single storage ring, with a proton beam and an antiproton beam circulating in opposite directions and colliding at six locations.

The important figure of merit of the collider is the luminosity, defined

as

$$
\mathscr{L}=f_{0} B \frac{N_{B}^{-} N_{B}^{+}}{4 \pi \sigma^{2}}
$$

with: $\quad f_{0}=$ revolution frequency

$$
B \quad=\text { number of bunches }
$$

$N_{B}=$ (anti) protons per bunch

$\sigma \quad=$ (gaussian) transverse bunch dimension

The luminosity varies during a run, decreasing if for no other reason than the loss of particles by collisions in the six interaction regions:

$$
\dot{N}_{\text {int }}=-6.0 \mathscr{L}_{\sigma}
$$

with $\sigma$ the total $\overline{p p}$ cross section. At $\mathscr{L}=10^{32} \mathrm{~cm}^{-2} \mathrm{~s}^{-1}$ and $\sigma=100 \mathrm{mb}$ for $20+20 \mathrm{TeV}$ we find

$$
\dot{N}_{\text {int }}=0.6 \times 10^{8} \text { per second }
$$


The figure of merit for physics experiments is the integrated or the average Juminosity

$$
\int_{0}^{\mathrm{T}} \mathscr{L}(\mathrm{t}) \mathrm{dt}=\langle\mathscr{L}\rangle \mathrm{T}_{\mathrm{c}},
$$

where the integration time $T_{c}$ is the antiproton collection time needed to accumulate enough antiprotons for injection to achieve the initial peak luminosity. The collection time is given by the antiproton accumulation rate $\dot{N}^{-}$according to

$$
\dot{N}^{-} T_{C}=B N_{B}^{-} .
$$

The collection time is not related to the luminosity lifetime $T_{\mathscr{L}}$, the latter being determined only by particle losses. Ideally one wants $\mathrm{T}_{\mathscr{L}}>\mathrm{T}_{\mathrm{C}}$;

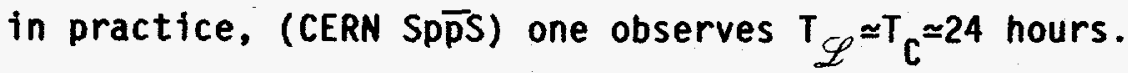

In this chapter we review the state of the art in antiproton production and accumulation, and we extrapolate techniques to potential application in a $\overline{p p}$ alternative to the SSC. We further discuss operational differences for the $\overline{\mathrm{pp}}$ collider, and sketch possible future developments.

The accelerator physics issues for a realistic $\overline{p p}$ collider with a luminosity of $10^{32} \mathrm{~cm}^{-2} \mathrm{~s}^{-1}$ are discussed in chapter 4 . 


\subsection{Antiproton Sources 2,3}

The evaluation of a potential antiproton source for a $\overline{p p}$ SSC option derives from three reference sources, either existing or expected to operate in the near future:

(1) The CERN Antiproton Accumulator (AA), operating since 1981 and providing practically all present experience of antiproton sources for colliders.

(2) The CERN Antiproton $\underline{C O L}$ lector (ACOL), designed as a pre-collector to the AA accumulator. ACOL operates at the repetition rate of the production synchrotron CPS and provides fast cooling prior to accumulation in AA. It is scheduled for operation in 1987.

(3) The FNAL antiproton source for the Tevatron I Project, initially tested in 1985 and expected to be operational in 1986. This source also consists of a two-ring scheme (debuncher/precooler ring and accumulator ring). It uses a high energy proton beam for antiproton production, and it collects antiprotons at $8.9 \mathrm{GeV} / \mathrm{c}$. With these parameters, the FNAL source is the one most suited for extrapolation to the SSC conditions.

Antiproton production rates $\bar{N}^{-}$are estimated according to the following principles.

(a) Antiprotons are produced by a proton beam impinging on a production target with a given repetition rate, proton momentum, and proton flux per pulse. The proton flux is limited only by the stability of the target. Repetition rate and proton momentum can be optimized within economical limits. 
(b) Antiproton production is described ${ }^{2}$ by the inclusive (invariant or laboratory) cross-section.

$$
\begin{aligned}
E \frac{d^{3} \sigma}{d p^{3}}=\frac{E}{p^{2}} \frac{d^{2} \sigma}{d p_{T} d \Omega} & \\
& =f\left(p^{*}, p_{T}, s\right),
\end{aligned}
$$

with $E, p=$ energy and momentum of the produced antiproton, $p^{*} \quad=$ the $\bar{p}$ (cms) momentum,

$\mathrm{p}_{\mathrm{T}} \quad=$ the $\overline{\mathrm{p}}$ (invariant) transverse momentum,

$S \quad=(\mathrm{cm} \text { energy })^{2}$.

Antiproton production data have been compiled for different incident and secondary momenta, and can be interpolated for different target materials. Reabsorption inside the target must be included. 2,4

(c) Antiproton yields ${ }^{4}$ are derived starting from the inclusive production cross section for the given incident and chosen secondary momentum, and integrating over the production distribution $\left(p^{*}, p_{T}\right)$ within the available transverse and momentum acceptances.

(d) Antiproton production rates $\dot{\mathrm{H}}^{-}$are scaled from the yield (per incident proton), applying the proton flux (per pulse) and repetition rate (per second).

Parameters and performances for the relevant antiproton sources are summarized in Table 3.1 . 


\subsection{Scenario for a $\bar{p} p$ Supercollider}

In the following we try to sketch a concept for the alternative components of the SSC as a $\overline{p p}$ collider.

(1) Compared to the injector for the $p p$ collider from the COR, the medium energy booster (MEB) would need to be upgraded to an energy of 150 $\mathrm{GeV}$ and a repetition rate of $1 \mathrm{~Hz}$ in order to optimize $\overline{\mathrm{p}}$ production. Antiprotons are selected at a secondary momentum of $10 \mathrm{GeV} / \mathrm{c}$, close to rest in the cms (Feynman $X=0$ ), and therefore at maximum production cross section. Figure 3.1 shows $\bar{p}$ production as a function of proton and antiproton momentum. Antiproton yield is more sensitive to the choice of the incident proton energy than to the choice of the secondary antiproton momentum.

(2) A proton flux of $1.25 \times 10^{12}$ protons per pulse is transported to a copper target. The flux is limited to this value by the instantaneous energy deposition in the target; energy dissipation leads to local heating and mechanical shock which are destructive if the load exceeds 200 Joules $\mathrm{gm}^{-1}$.

(3) Antiprotons are collected from the target with momentum $10 \mathrm{GeV} / \mathrm{c}$, momentum acceptance $|\Delta p / p|=0.06$, and transverse acceptance

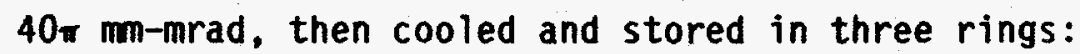

- the $10 \mathrm{GeV} / \mathrm{c}$ debuncher/precooler ring operating at $1 \mathrm{~Hz}$ synchronously with the MEB;

- the $10 \mathrm{GeV} / \mathrm{c}$ accumulator ring for ultimate cooling and stacking of antiprotons over about 30 seconds; 


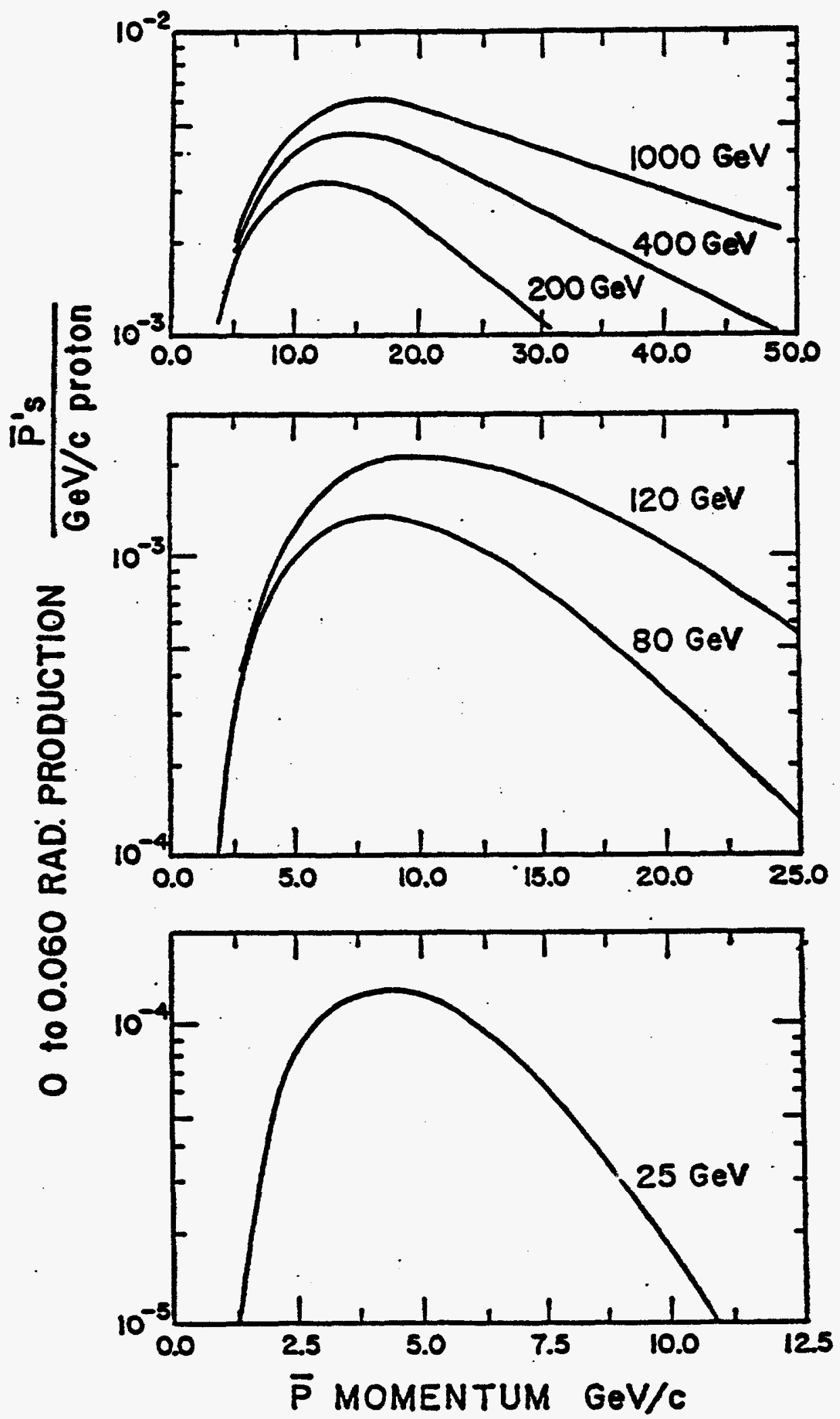

Fig. 3.1 Relative antiproton production for different incident proton momentum as indicated. 
- the $150 \mathrm{Gev} / \mathrm{c}$ holding ring for cooled antiprotons, after intermediate acceleration in the MEB. The purpose of this ring is to store the cooled stack and allow use of the injector complex for test beams. The figure of $150 \mathrm{GeV}$ is not the result of optimization, but was chosen assuming the ring would be placed in the same tunnel as the MEB. The cooling requirements, especially from the precooler, are greater than in the FNAL source, owing to increased acceptance and decreased cooling time. The required bandwidth and cooling power are considered to be well within present possibilities.

(4) Protons from the MEB and antiprotons from the holding ring are separately accelerated in the HEB and injected into the main collider ring. The modified specifications of the main ring require in particular:

- an injection scheme to transfer both beams into the same ring; - an electrostatic separation of the two beams to limit the consequences of beam-beam forces and reduce resulting orbit distortions and beam losses;

- a magnet bore radius of $2.81 \mathrm{~cm}$ (as compared to $2.00 \mathrm{~cm}$ in the COR) to accept the two helically separated beams.

(5) Six interaction regions, together with injection and abort, are evenly distributed about the circumference of the ring to present an eightfold symmetry as shown in Fig. 3.2. The reason for this "distributed" scheme (in contrast to the "clustered" scheme of the CDR) is the extra dispersion introduced by the electrostatic separation scheme. 


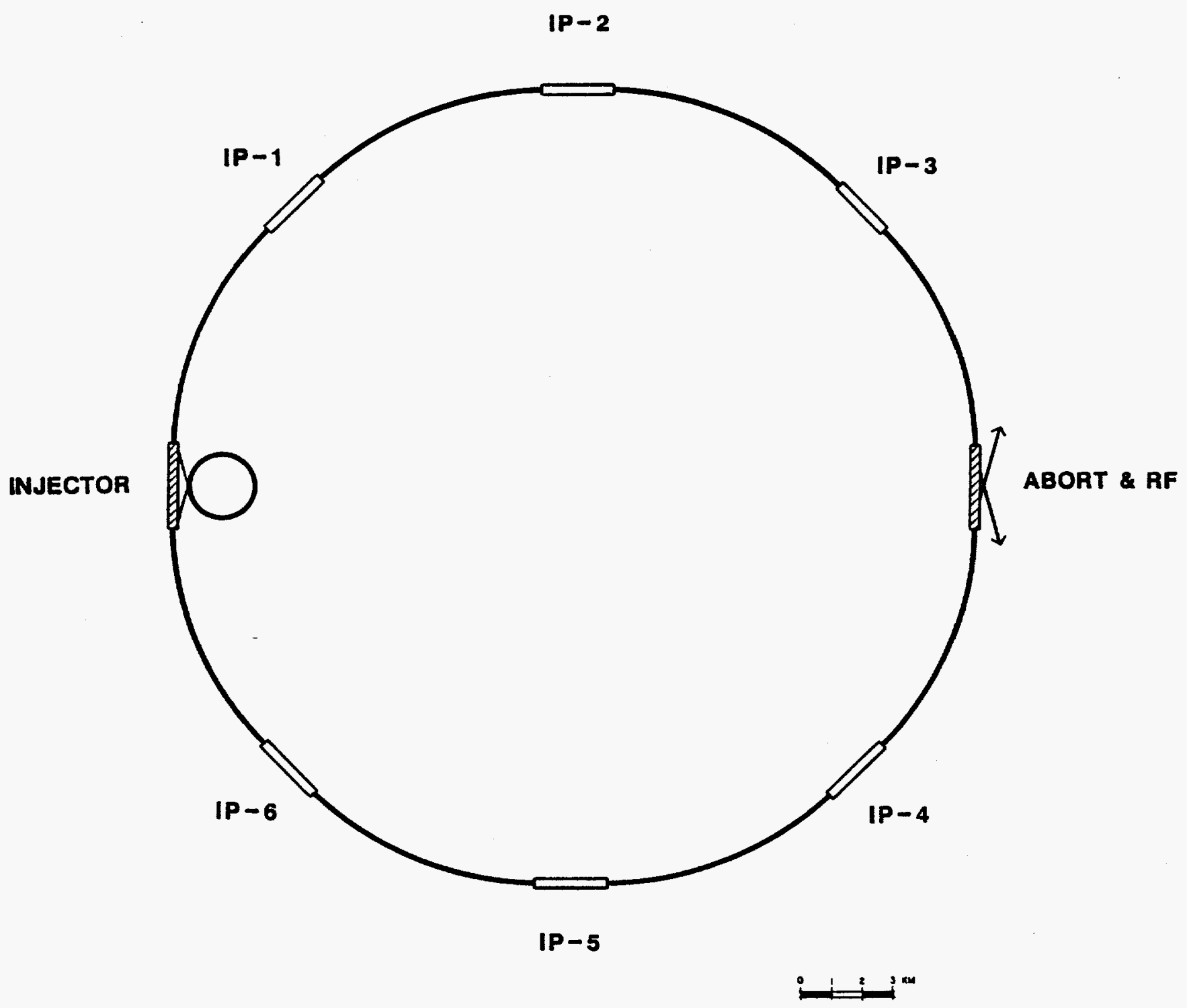

Fig. $3.2 \overline{p p}$ Collider Layout. 


\subsection{Operational Aspects}

The sensitivity of a physics experiment at a collider is determined by the number of events per unit cross section

$$
N_{\mathrm{ev}} / \sigma=\int_{0}^{T} \mathscr{L}(\mathrm{t}) \mathrm{dt}=\langle\mathscr{L}\rangle \mathrm{T}
$$

The time dependence of the luminosity is given by the loss of particle density of the stored beams through beam-beam interaction and particle collisions. In the case of a pp collider, integrated and average luminosities can be optimized through appropriate restoration of the circulating beams by dumping and reinjection. In the case of a $\overline{p p}$ collider, reinjection is limited by the antiproton collection time $T_{C}$ so that

$$
N_{e v} / \sigma=\int_{0}^{T} \mathscr{L}\left(T_{\mathscr{L}}, \mathrm{t}\right) \mathrm{dt}=\left\langle\mathscr{L}\left(\mathrm{T}_{\mathscr{L}}\right)\right\rangle \mathrm{T}_{\mathrm{c}}
$$

It is conceivable that the luminosity lifetime $\mathrm{T}_{\mathscr{L}}$ and the antiproton collection time $T_{c}$ are so mismatched that the optimum refill period cannot be reached.

Apart from the question of optimal refilling, the limited availability of antiprotons will occasionally lead to non-recovery in the case of accidental beam losses, causing an increase in the down-time of the $\overline{p p}$ collider compared to the pp option.

There are several operational differences which affect the reliability of the $\overline{p p}$ collider.

(1) In addition to three proton booster rings, there are three additional rings in the antiproton source, with many additional extraction and injection operations. The three source rings present operational difficulties because of the delicate storing and cooling procedures. 
(2) The injection of both protons and antiprotons into the same collider main ring requires an injection scheme of greater complexity than for the two-ring collider of the CDR.

(3) the storage of both proton and antiproton beams in the same collider ring requires a separation scheme to minimize the effects of beam-beam interactions. This presents technical complications from the presence of electrostatic separations, and it adds to the operational complexity.

We cannot predict the operational efficiency of a $\overline{p p}$ SSC. Instead, we quote the empirical values obtained after five years of collider operation at the CERN SpDSS (Table 3.2). The principal conclusions are (averaged over 1984/85):

$$
\begin{aligned}
& \frac{\text { hours realized }}{\text { hours scheduled }}=\frac{\mathrm{hr}}{\mathrm{hs}}=1 / 2 \\
& \frac{\text { average luminosity }}{\text { peak luminosity }}=\frac{\mathrm{aL}}{\mathrm{pL}}=1 / 3
\end{aligned}
$$

To a large extent the efficiencies are independent and therefore multiplicative. Further

$$
\left\langle T_{\mathscr{L}}\right\rangle \simeq\left\langle T_{c}\right\rangle \simeq 28 \mathrm{~h} .
$$

It must be kept in mind, however, that these efficiency ratios are not unity for a pp collider, because of refill and accidental losses, and luminosity decay. We again quote empirical figures for the CERN ISR: at $31 \mathrm{GeV}$, the ISR has achieved 0.86 for $\mathrm{hr} / \mathrm{hs}$ and 0.6 for aL/pL (Table 3.3). In addition, the luminosity lifetime for the ISR was 60 hours. 
Table 3.2

CERN Sp $\overline{p S}$ Operation 1981-1985

\begin{tabular}{llllll} 
Operational Features & 1981 & 1982 & 1983 & 1984 & 1985 \\
\hline Beam Energy (GeV) & 273 & 273 & 273 & 315 & 315 \\
Beta $\left(\mathrm{m}^{2}\right)$ & 2 & 1.5 & 1.3 & 1.0 & 1.0 \\
Beta $_{V}\left(\mathrm{~m}^{2}\right)$ & 1 & 0.75 & 0.65 & 0.5 & 0.5 \\
\hline
\end{tabular}

Int. Luminosity $\left(\mathrm{nb}^{-1}\right)-$ Average values

\begin{tabular}{|c|c|c|c|c|}
\hline i) per run & & 0.5 & 2.1 & 5.3 \\
\hline ii) per day & & 0.4 & 1.8 & 5.1 \\
\hline iii) per year & 0.2 & 28 & 153 & 395 \\
\hline
\end{tabular}

Luminosity $\left(10^{29} \mathrm{~cm}^{-2} \mathrm{~s}^{-5}\right)$
i) peak
0.5
1.7
3.5
3.9
ii) average per run
0.1
0.5
1.0
1.5

$\begin{array}{llllll}\text { A) Hours Scheduled } & 698 & 1750 & 2064 & 2136 & 2694 \\ \text { B) Hours Realized } & 140 & 748 & 889 & 1065 & 1286 \\ \text { Number of runs } & 14 & 56 & 72 & 77 & 78 \\ \text { Average run duration (h) } & 10 & 13 & 12 & 15 & 17 \\ \text { \% of run termin. by faults } & & 41 & 40 & 32 & 15\end{array}$


Table 3.3

ISR Statistics for 1983

\begin{tabular}{|c|c|c|c|c|c|c|c|c|c|c|c|c|c|c|c|c|c|c|c|}
\hline $\begin{array}{c}\text { ENERGY } \\
\text { Gev/C }\end{array}$ & \multicolumn{2}{|c|}{$\begin{array}{c}31 L B \\
\text { P.P }\end{array}$} & \multicolumn{2}{|c|}{$\begin{array}{c}26 D L \\
P . P\end{array}$} & \multicolumn{2}{|c|}{$\begin{array}{l}26 D L \\
\text { P. } \bar{P}\end{array}$} & \multicolumn{2}{|c|}{$\begin{array}{c}22 L B \\
\text { P.P }\end{array}$} & \multicolumn{2}{|c|}{$\begin{array}{l}\text { 15LB } \\
\text { P.P }\end{array}$} & \multicolumn{2}{|c|}{$\begin{array}{l}\text { R } 210 \\
26 \mathrm{FP} \\
31 T W\end{array}$} & \multicolumn{2}{|c|}{$\begin{array}{c}\text { R } 704 \\
3.5 \mathrm{Gev} \\
\mathrm{P} / \overline{\mathrm{p}} / \text { jet }\end{array}$} & \multicolumn{2}{|c|}{$\begin{array}{c}\alpha \text { 's } \\
\text { deutter. } \\
22 / 31 L B\end{array}$} & $\begin{array}{l}\text { Machine } \\
\text { Prepar. }\end{array}$ & \multicolumn{2}{|c|}{ TOTAL } \\
\hline \multirow{3}{*}{$\begin{array}{l}\text { HOURS } \\
\text { SCHEDULED }\end{array}$} & $\boldsymbol{F}$ & $P$ & $F$ & $P$ & $\mathbf{F}$ & $P$ & $F$ & $P$ & $F$ & $P$ & $F$ & $\mathbf{P}$ & $F$ & $P$ & $F$ & $P$ & 77 & Fill. & Phys. \\
\hline & 572 & 3126 & 31 & 152 & 3 & 412 & 27 & 101 & 22 & in & 12 & 28 & 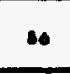 & sst & 40 & 2as & $E=1$ & $\omega$ & דים \\
\hline & \multicolumn{2}{|c|}{3698} & \multicolumn{2}{|c|}{183} & \multicolumn{2}{|c|}{485} & \multicolumn{2}{|c|}{128} & \multicolumn{2}{|c|}{136} & \multicolumn{2}{|c|}{46} & \multicolumn{2}{|c|}{645} & \multicolumn{2}{|c|}{325} & 123 & \multicolumn{2}{|c|}{5769} \\
\hline \multirow{3}{*}{$\begin{array}{l}\text { HOURS } \\
\text { RUNNING }\end{array}$} & $F$ & $p$ & $F$ & $P$ & $\boldsymbol{F}$ & $p$ & $F$ & $\mathbf{P}$ & $F$ & $P$ & $F$ & $P$ & $F$ & $P$ & $\boldsymbol{F}$ & $p$ & & Fil. & Phys. \\
\hline & s2n.6 & 2702.9 & 22.2 & |W7.2 & $\infty$ & 408 & 21.6 & 02.2 & 20.1 & ט & 7.4 & ses. & 109 & $s 7.8$ & Sas & isoses & & ones & SIms \\
\hline & \multicolumn{2}{|c|}{3285.5} & \multicolumn{2}{|c|}{169.5} & \multicolumn{2}{|c|}{472.6} & \multicolumn{2}{|c|}{107.6} & \multicolumn{2}{|c|}{1024} & \multicolumn{2}{|c|}{159} & \multicolumn{2}{|c|}{566.8} & \multicolumn{2}{|c|}{2013} & 958 & \multicolumn{2}{|c|}{ sonod } \\
\hline $\begin{array}{l}\text { TIME } \\
\text { LOST }\end{array}$ & \multicolumn{6}{|c|}{$18 \mathrm{KV} \longrightarrow 193 \mathrm{H}$} & \multicolumn{4}{|c|}{ MPS $\longrightarrow 137 \mathrm{H}$} & & & \multicolumn{4}{|c|}{ ISR $\longrightarrow 392.6 \mathrm{H}$} & $6 H=$ & $2.6 \mathrm{HC}$ & DURS \\
\hline
\end{tabular}

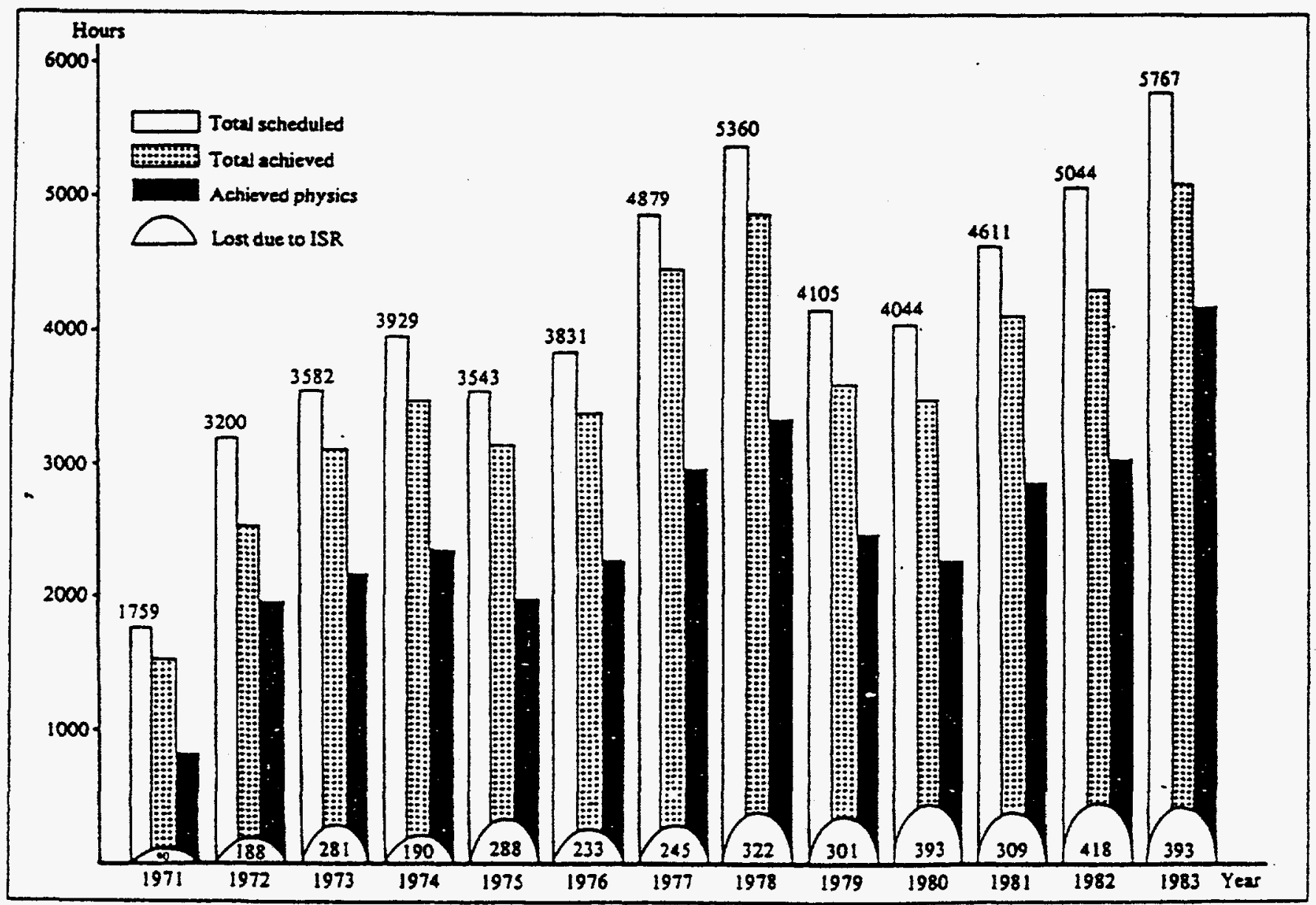




\subsection{Advanced Schemes}

The present assessment is restricted to techniques and components which either work or exist at CERN or FNAL or are safely extrapolated from those facilities. The performance of the antiproton accumulation system is limited to (Table 3.1)

$$
\dot{N}^{-}=3 \times 10^{8} \mathrm{~s}^{-1}
$$

and the luminosity of the SSC $\overline{p p}$ collider cannot exceed $\mathscr{L}=10^{32} \mathrm{~cm}^{-2} \mathrm{~s}^{-1}$

Several methods have been discussed ${ }^{3}$ which, after appropriate development work, may push up luminosities by a significant factor.

(1) At the given target flux of $1.25 \times 10^{12}$ protons per pulse, use of a conducting target may increase the acceptance. At present, it seems that the focusing power obtained is of little effect at $10 \mathrm{GeV}$ antiproton energy.

(2) The limitation for the fluxes under consideration is not incident proton supply but heat dissipation in the target. Local dissipation can be reduced by the use of "moving targets" (or "multiple targets"). By moving the incident proton beam, and synchronous ly the secondary antiproton beam, over the target, the heat deposit is distributed. Clearly this cannot easily be made compatible with method (1).

(3) Higher antiproton production necessitates larger cooling power, since $I / \tau$ \& $W / N$. Technical limitations are mainly in bandwidth $(W)$ and in the combination of large band width with high power. The solution to this problem lies either in technological advance in the RF domain, or in a shift of the problem to the logistic domain by the use of 
multiple (parallel or sequential) cooling rings, with reduced bandwidth or particle number per ring.

Developments in these fields are likely, but their success and schedule cannot be predicted. Any reliance on the above techniques in the design of a pp collider would add both time and cost to the project and therefore are not considered in this report. 
References

1. Conceptual Design of the Superconducting Super Collider, SSC-SR-2020, SSC Central Design Group, March 1986.

2. see for example the contributions of J.V. Allaby and E. Jones in CERN Accelerator School 1984, CERN 84-15.

3. R.P. Johnson and J. Simpson et a1. $\bar{p}$ Source Working Group, Chicago Workshop on $\overline{p p}$ Options for the Supercollider (1984).

4. C. Hojvat and A. Van Ginneken, Nuc 1. Instr. Meth. 206, 67 (1983).

5. Design Report Tevatron I Project, Fermi National Accelerator Laboratory (1983). 


\section{Chapter 4}

\section{MACHINE ISSUES}

\subsection{Introduction}

An important argument for the proton-antiproton SSC is the fiscal advantage, since only one ring of superconducting magnets is needed. This fiscal advantage is counterbalanced to some extent by the need for an antiproton source, and by the need for an increase in magnet aperture to accommodate the separation of the two multi-bunch beams in the same vacuum chamber. Further, the added complexity of the operation, which comes with the addition of antiproton production and the separation of the two beams, will reduce reliability.

Section 4.2 describes the characteristics of an antiproton source which would provide c00l antiprotons at a rate high enough to allow a luminosity of $10^{32} \mathrm{~cm}^{-2} \mathrm{~s}^{-1}$ to be achieved. In section 4.3 the main ring parameters for this nominal luminosity are derived, but in addition different scenarios, such

as the consequences of trying to attain a luminosity of $10^{33} \mathrm{~cm}^{-2} \mathrm{~s}^{-1}$, are considered. An estimation of the required magnet aperture increase is made, after a discussion of the various separation schemes which have been proposed. Current limitations are shown to be less important than those noted in the Conceptual Design Report. 


\subsection{Antiproton Source}

\subsubsection{Introduction}

Several antiproton sources for $\overline{p p}$ collisions in the SSC have been proposed in the past. ${ }^{1,2}$ These have been based upon the $\overline{p p}$ source designs used at CERN and Fermi lab and have been scaled up in number of source rings, stochastic cooling systems and frequencies, and final accumulation rates. In this section, we have taken the basic design presented at the University of Chicago workshop and have adapted it to the specific needs of this $\bar{p} p$ collider design. The cooling calculations and stochastic systems proposed at Chicago have been accepted, while the ring designs have been changed in order to conform to the present SSC design as given in the Conceptual Design Report. In addition, the scope of the $\bar{p}$ source has been scaled down to reflect the presently needed accumulation rate. The design luminosity of $10^{32} \mathrm{~cm}^{-2} \mathrm{~s}^{-1}$ requires an replenishing rate of $\sim 2 \times 10^{8} \bar{p}^{\prime}$ s per second.

\subsubsection{Parameters}

The antiproton source design presented here is capable of an accumulation rate of $3 \times 10^{8} \bar{p}^{\prime}$ s per second, assuring a usable average rate of $2 \times 10^{8} \mathrm{~s}^{-1}$ after losses, inefficiencies, et cetera. The source consists of a target, a $10 \mathrm{GeV} / \mathrm{C}$ Debunching Ring, a $10 \mathrm{GeV} / \mathrm{C}$ Accumulator Ring and a $150 \mathrm{GeV} / \mathrm{c}$ Storage Ring in addition to the SSC LOW, Medium and High-Energy Boosters. Modifications have been made to the MEB from the CDR. The parameters of the modified SSC booster complex are given in Table 4.1. The parameters of the new $\bar{p}$ source rings are listed in Table 4.2 and the cooling and accumulation parameters are given in Table 4.3. 
Table 4.1

Injector Characteristics

\begin{tabular}{llll} 
Momentum/GeV/c: Top & $\frac{\text { LEB }}{8.0}$ & $\frac{\text { MEB }}{150}$ & $\frac{\text { HEB }}{1000}$ \\
\multicolumn{1}{c}{ Injection } & 1.2 & 8 & 150 \\
Circumference/m & 249.6 & 2688 & 6000 \\
Normalized emittance/rmm-mrad & 0.75 & 0.83 & 0.91 \\
Cycle time/sec & 0.1 & 1 & 60 \\
rf frequency/MHz & 62 & 62.5 & 62.5 \\
Harmonic number & 52 & 560 & 1250
\end{tabular}

Table 4.2

(a) Antiproton Parameters

$\begin{array}{ll}\text { Proton Momentum } & 150 \mathrm{GeV} / \mathrm{c} \\ \bar{p} \text { bunch spacing } & 4.80 \mathrm{~m} \\ \text { Pulse length } & 2 \times \mathrm{LEB}=500 \mathrm{~m} \\ \overline{\mathrm{p}} \text { momentum } & 10 \mathrm{GeV} \\ \overline{\mathrm{p}} \text { bunch spacing } & 4.779 \mathrm{~m}\end{array}$

(b) Antiproton System Parameters

Quantity Debuncher Accumulator Storage Ring

$\begin{array}{llll}\text { Momentum/GeV/C } & 10 & 10 & 150 \\ \text { Circumference/m } & 573.5 & 528 & 2688 \\ \text { RF wavelength/m } & 4.779 & 4.8 & 4.8 \\ \text { Harmonic number } & 120 & 110 & 560\end{array}$


Table 4.3

Antiproton Production Characteristics

Step 1: $\bar{p}$ Production

Yield $\bar{p} / p$

Momentum

Acceptance: $d p / p$

Cycle time

transverse

Target

Focusing

Step 2. Debunching

Final momentum spread

RF voltage

Cycle time

Step 3. Transverse Precooling

Final emittance

Bandwidth

Power

Cycle time

Step 4. Transverse Cooling

Final transverse emittance

Bandwidth

Power

Cycle time

Step 5. Longitudinal Precooling

Final momentum spread

Cooling system: Frequency band

Schottky power

Total Power

Cycle time

P.U. noise temperature

Step 6. Stochastic stacking

Longitudina 7 : Initial density

Final density

Bandwidth

Power

Transverse: Final emittance Bandwidth

Cycle time

Power

Step 7. $\bar{p}$ Storage in last Booster

Storage momentum

Transfer rate

Time to fill
$3 \times 10^{8 / 1.25} \times 10^{12}$

$10 \mathrm{GeV} / \mathrm{C}$

$6 \%$

$40 \pi m m-m r$

$1 \mathrm{~s}$

Copper

Lithium lens

$0.5 \%$

IOMV

15

$20 \pi m m-m r$

4-8 GHz

$10 \mathrm{~kW}$

is

$3 \pi m m-m r$

4-8 GHz

$10 \mathrm{~kW}$

75

$0.15 \%$

4-8 GHz

$2500 \mathrm{~W}$

$3700 \mathrm{~W}$

$300 \mathrm{~K}$

is

$20 p / e V$

$3000 \mathrm{p} / \mathrm{eV}$

4-8 GHz

$10 \mathrm{~kW}$

$<1 \pi m m-m r$

4-8 GHz

$<7 \mathrm{~kW}$

$40 \mathrm{~s}$

$150 \mathrm{GeV} / \mathrm{c}$

$40 \mathrm{~s}$

22 hours 


\subsubsection{Machine Requirements}

Linac and Low Energy Booster. The linac and LEB remain as described in the CDR. They must deliver $20 \%$ more intensity to the MEB than in the CDR but we have assumed that this will not require any changes.

Medium Energy Booster. The MEB has to be extensively modified. In order to achieve the required $\bar{p}$ accumulation rates, the MEB cycle time must be reduced from $4 \mathrm{~s}$ to $1 \mathrm{~s}$, and in addition the top energy must be increased from 100 to $150 \mathrm{GeV} / \mathrm{c}$. These changes require more $r f$ and power supplies as well as more cooling. The magnets remain unchanged, although their number increases by roughly 50\%. In order to achieve $150 \mathrm{GeV} / \mathrm{c}$ and match into the entire cooling scenario, the MEB circumference must increase to $2688 \mathrm{~m}$ from $1900 \mathrm{~m}$. In addition, the optics of the MEB will be changed to reduce the maximum dispersion from its present value of $14 \mathrm{~m}$ to a more reasonable value, approximately $3.5 \mathrm{~m}$. This will allow the more intense batches to fit within the present magnetic aperture. Transition crossing will not be a problem.

High Energy Booster. The HEB remains as described in the CDR.

Target Station. A target station similar to that at Fermilab will be required, with a simple copper target followed by a lithium lens collection system and a pulsed dipole beam deflector. Improvements such as a focusing lithium lens, a current carrying target or sweeping magnets may be added to improve the yield, but they are not needed for this design.

Debuncher Ring. A new Debuncher Ring, running at $10 \mathrm{GeV} / \mathrm{c}$, must be built. It can be scaled from the Fermilab design and will have a circumference of $573.5 \mathrm{~m}$. The lattice will be similar to the Fermilab design, having $12 \%$ more normal cells in order to reach the higher energy with the same magnetic fields. The magnet apertures must increase in order to have a transverse acceptance of $40 \pi$ in both planes and $6 \%$ momentum acceptance, instead of $30 \pi$ and $4 \%$ as in the Fermilab Debuncher. The rf systems have to increase by 
50\% to accommodate the larger momentum bite. The Debuncher will have a stochastic cooling system, as specified in the Chicago Workshop, in order to do fast transverse precooling.

Accumulator Ring. Again, a $10 \mathrm{GeV} / \mathrm{C}$ Accumulator Ring, which can be scaled from the Fermilab design, must be built. Here the apertures need not be increased, only the total magnetic fields and circumference, which must be $528 \mathrm{~m}$ in order to match onto the source system. The lattice design will need to be somewhat modified in order to obtain an appropriate mixing factor. Otherwise, the design will be similar to that of Fermilab, but with longer magnets. The accumulator must have several different stochastic cooling systems: as described in the Chicago design, there will be four systems, for transverse cooling, longitudinal precooling, momentum stacking and accumulation, and core betatron cooling.

Storage Ring. The largest addition to the SSC design required for an appropriate $\bar{p}$ source is of a $150 \mathrm{GeV} / \mathrm{c}$ holding ring. This will store and accumulate the cooled $\bar{p}$ 's from the Accumulator Ring, in boxcar style and in momentum, for approximately a day, to achieve the total intensity of $1.5 \times 10^{13}$ needed for collisions. This will be a simple dc machine made of f0D0 cells, matched in geometry and circumference to the MEB and sharing the MEB tunnel.

\subsubsection{Antiproton Production and Accumulation}

$\overline{\mathrm{D}}$ Production. The LEB is used to accelerate protons to $8 \mathrm{GeV} / \mathrm{C}$ and to inject them into the MEB with a cycle time of $0.1 \mathrm{~s}$. In order to achieve the needed production rate, the LEB must deliver $1.2 \times 10^{10}$ protons per bunch. This represents an increase of $20 \%$ over the CDR but will not require any changes to be made to the LEB. Two batches of 104 bunches will be loaded into the MEB, which will then accelerate the protons to $150 \mathrm{GeV} / \mathrm{c}$ in $1 \mathrm{~s}$. At 150 
$\mathrm{GeV} / \mathrm{c}$, the MEB RF voltage will suddenly be increased to mismatch the bunch and the bucket. The bunches will rotate by $90^{\circ}$, in $1 / 4$ of a synchrotron period, When they will be then extracted and sent on to the copper target. A total of $1.25 \times 10^{12}$ protons per second thus hit the target, producing $\bar{p}$ 's which are captured at $10 \mathrm{GeV} / \mathrm{c}$ into a $6 \%$ momentum bite and into a transverse acceptance of $40 \pi \mathrm{mm}$-mrad in both planes. This has been calculated to give a capture rate of $3 \times 10^{8}$ antiprotons per second. 3

Debuncher. In the Debuncher Ring, the $\bar{p}$ 's are captured into mismatched rf buckets and rotated by $90^{\circ}$. The $r f$ system is then turned off adiabatically. This reduces the momentum spread from $6 \%$ to $0.5 \%$. The $\bar{p}^{\prime} s$ undergo a 4-8 $\mathrm{GHz}$ transverse precooling which in one second reduces the transverse emittance by a factor of two. At this point, the beam is extracted from the Debuncher and transferred into the Accumulator Ring.

Accumulator. Beam is injected into the Accumulator once every second. Since the actual injection, capture and movement to the stacking orbit takes only a fraction of a second, there is ample time to cool the beam somewhat while it is on the injection orbit. The transverse emittance could not be cooled efficiently while in the Debuncher because of the poor mixing factor, a consequence of the $r f$ debunching requirements. In the Accumulator, however, the mixing factor will be very good, so the one second period between injected pulses will be used for both transverse cooling and longitudinal precooling. This will reduce the beam to a transverse emittance of $\sim 3 \pi$ and leave it with a full momentum spread of $0.15 \%$. Before the next pulse is injected, the beam will be captured by a small rf system, moved over to the stacking orbit and deposited in the tail of the stack.

A high-power 4-8 GHz momentum stochastic cooling system will then be used to accumulate the $\bar{p}^{\prime}$ s into a dense core. The $\bar{p}$ density will have increased 
from $20 / \mathrm{eV}$ to $3000 / \mathrm{eV}$ after approximately 40 seconds. The beam will also be subjected to a low-power core betatron cooling system during the stacking cycle, to produce a final emittance of $\sim 1 \pi$. At the end of this process, the dense core of the beam will be adiabatically csptured by the rf system, moved to the extraction orbit, and reinjected back into the MEB, which will by then have been switched out of its production-mode cycle. The beam, with a population of $1.2 \times 10^{10} \overline{\mathrm{p}} \mathrm{s}$ and a density of $3000 / \mathrm{eV}$, will then be accelerated to $150 \mathrm{GeV} / \mathrm{c}$, and transferred into the storage Ring. The $\overline{\mathrm{p}}$ production mode will then resume.

Storage Ring. The Storage Ring will receive an Accumulator pulse every 40 seconds, to be loaded with a combination of boxcar stacking and momentum stacking. After five pulses are loaded boxcar style in 200 seconds, the beam will be captured by the rf system, moved over to a stacking orbit, and deposited on the bottom of the momentum stack. This process will be repeated for a total of 1700 pulses to achieve the final desired intensity of $1.5 \times 10^{13}$, assuming a total efficiency of $65 \%$ from the target to the final storage ring, and a momentum stacking efficiency of $85 \%$. The energy spread of each momentum stack will be about $4 \mathrm{MeV}$ and the final stacked beam will have an energy spread of 1\%. Allowing some additional aperture for beam transfer, etc., the required momentum aperture will be $1.25 \%$.

At this point, when the $\bar{p}$ density is be approximately $12500 / e V$, beam will be extracted from the Storage Ring and injected into the SSC with a longitudinal emittance of $0.035 \mathrm{eV}-\mathrm{s}$. A suppressed-bucket rf system in the storage ring will capture bunches of the required $6.4 \times 10^{9}$ antiprotons in $375 \mathrm{MHz}$ buckets, with a bunch spacing of $40 \mathrm{~m}$. One full turn of the storage ring will allow approximately 67 such bunches to be transferred by a single turn shuttered kicker extraction system. 


\section{$\overline{\mathrm{D}}$ Injection}

In order to achieve the specified design parameters, the Main Ring must be loaded with about 2400 proton and antiproton bunches, with a separation of $40 \mathrm{~m}$ ( 120ns). In any loading scheme it is desirable to avoid a lengthy injection cycle to minimize the amount of time spent at the injection energy, where beam lifetimes may prove to be short. In this section we outline a possible injection scenario which does not require a significant change from the protonproton case.

We shall assume that the collider ring is loaded first with protons at the appropriate bunch spacing. During this time, the closed orbits are adjusted and any other set-up procedures are done before the antiproton accumulation is terminated. As well as the increased bunch spacing, we require that the circulating abort gap in the proton beam be increased to $\sim 10 \mu \mathrm{s}$ from the $3 \mu \mathrm{s}$ used in the pp collider. Once the circulating proton beam has been established the antiproton transfers are started.

At full turn on about 67 bunches are transferred from the storage ring to the HEB. This bunch train is 10ps long and fills half the HEB. The antiprotons are accelerated in the HEB and then transferred as a single batch into the collider into the abort gap in the circulating proton beam. During the 60 s needed to cycle the HEB with another batch of antiprotons, the freshly transferred antiprotons are rotated azimuthally with respect to the proton beam by the $r f$ system, so that the 10us abort gap is re-established before the next transfer takes place. The antiprotons are thus loaded boxcar fashion in a similar way to the protons, the only difference being that twice the number of batches are needed since only half the circumference of the HEB is used on each transfer. In this way the need to interleave 2400 individual bunches is avoided and only 36 antiproton injection cycles are necessary. The injection 
kicker rise time is defined by the bunch spacing $(-120 \mathrm{~ns})$, and the fall time is specified by the time difference between the abort gap and the length of the antiproton batch. 


\subsection{Main Ring}

\subsubsection{Introduction}

The parameters of the main ring of a proton-antiproton SSC are derived in this section. The derivation is based on the work which began with the 1984 DPF workshop in Chicago ${ }^{1}$ and continued at the Snowmass Summer Study ${ }^{2}$ of the same year. Only aspects peculiar to the proton-antiproton option are considered here, all general parameters being taken from the Conceptual Design Report for the proton-proton SSC. ${ }^{4}$ A nominal luminosity of $10^{32} \mathrm{~cm}^{-2} \mathrm{~s}^{-1}$ is taken, but other scenarios, with different performance parameters, are also considered.

The most crucial quantities to be derived are the antiproton production rate required to achieve a given luminosity, and the beam separation needed to reduce the close encounter beam-beam forces. General parameters, such as the number of bunches, the bunch population, and the number of events per interaction, are derived in section 4.3.2, and are used to define the necessary antiproton production rate.

This leads to a discussion of the technical issues in sections 4.3 .3 to 4.3.7. An overview is given of the several separation methods which have been proposed, leading to an estimate of the required orbit separation and a calculation of the required magnet aperture increase. Current limitations are briefly considered, and shown not to be critical in the nominal case. 


\subsubsection{Parameters}

The performance of the proton-antiproton storage ring can be characterized by the luminosity

$$
\mathscr{L}=\frac{f_{0}}{4 \pi} \frac{B N_{B}^{-} N_{B}^{+}}{\left(\varepsilon B^{*} / Y\right)}
$$

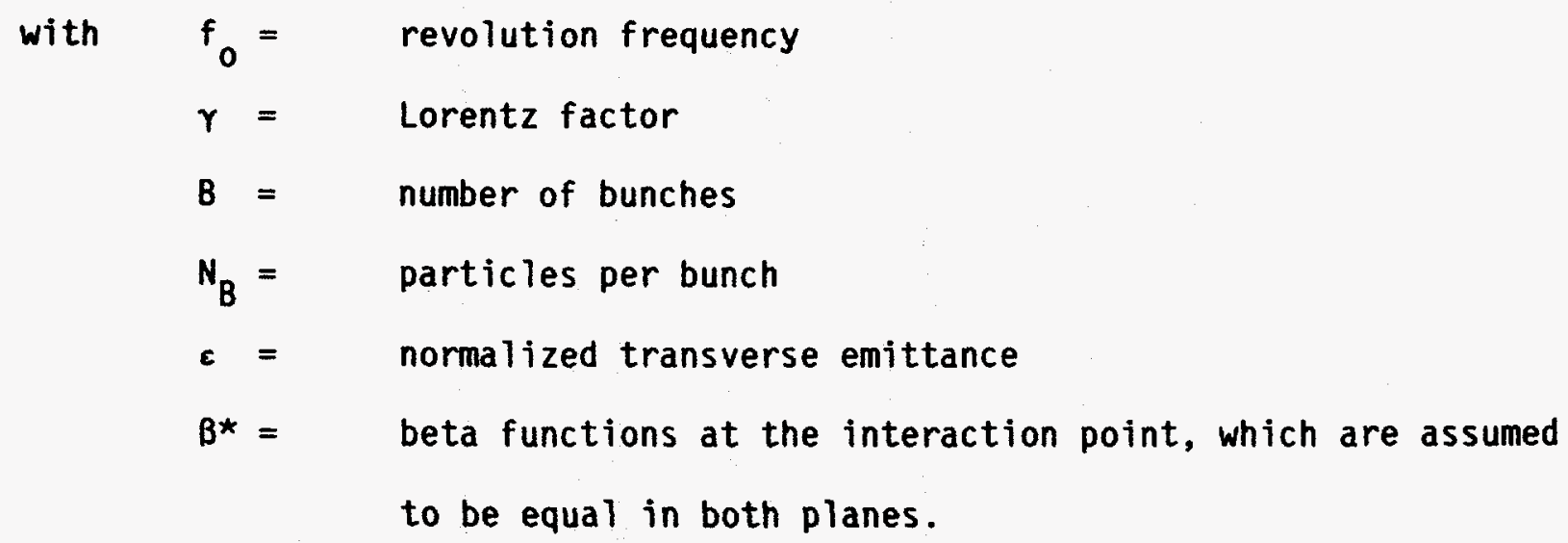

In all subsequent considerations, the emittances for the proton and antiproton beams are assumed to be $\varepsilon=10^{-6}$ meters, which sets certain requirements on the injection scheme. Beta values of $1 \mathrm{~m}$ and $0.5 \mathrm{~m}$ at the interaction point were used for the calculations. If the antiprotons are cooled down to the nominal normalized emittance after a collection time $T_{c}$, the total number of antiprotons available is given by

$$
N^{-}=B N_{B}^{-}=\dot{N}^{-} T_{C}
$$

A cycle time of 24 hours and a collection time of 22 hours have been assumed in what follows.

After substituting the limitation defined by (2) in equation (1), and expressing the proton bunch current by the head-on beam-beam tune shift

$$
\xi=\frac{r_{p}}{4 \pi} \frac{N_{B}^{+}}{c},
$$


we get for the luminosity

$$
\mathscr{L}=\frac{f_{0} Y T_{c}}{r_{p^{*}} \dot{N}^{-} \xi} .
$$

or

$$
\mathscr{L}\left[10^{32} \mathrm{~cm}^{-2} \mathrm{~s}^{-1}\right]=0.645 \mathrm{~N}^{-}\left[10^{8} \mathrm{~s}^{-1}\right] \xi\left[10^{-3}\right]
$$

In this parameterization, the attainable luminosity depends only on the antiproton production rate and the tune shift parameter. A circumference of 96 kilometers is assumed, corresponding to the simple SSC test lattice used in further calculations below; this value is somewhat larger than the value anticipated in the CDR. This minor disparity is partially justified by the recommendation of this report, explained below, that a lattice with distributed interaction regions is preferable for proton-antiproton operation.

Another restricting parameter which must be controlled is the average number of events per crossing,

$$
<n>=\mathscr{L}_{\sigma_{i}} \frac{S_{B}}{c}=\frac{4 \pi \varepsilon Y}{B^{\star} r_{p}} \sigma_{i} \xi^{2}=1.27 \xi^{2}\left[10^{-6}\right],
$$

with $S_{B}$ the bunch distance and $\sigma_{j}$ the inelastic cross section, taken to be $120 \mathrm{mb}$ at $20 \mathrm{TeV}$. Expression (5) is valid only for equal bunch populations. It should be noted that the number of events per crossing increases in proportion to the product of the bunch currents. Thus for constant luminosity, <n> can be reduced by increasing the number of bunches while decreasing the antiproton bunch population to keep the total number of antiprotons constant, and by keeping the proton bunch population constant.

This leads to unequal proton and antiproton currents. Since the multibunch beams are circulating in the same vacuum chamber, they influence each other with forces proportional to the number of particles per bunch. The tune 
shifts produced by this effect must be compensated if they are above a tolerable limit. If the currents of the two interacting bunches are not equal, the difference of the tune shifts must be compensated by magnetic multipoles which generate a quadrupole feed-down proportional to the sign of the orbit displacement. Since these nonlinear multipoles reduce the dynamic aperture, it is preferable to avoid using them by keeping the current per bunch equal for both beams. This can be done by adjusting the number of bunches, which according to equation (4) is still a free parameter. Using equations (1) and (2) we get as a condition for equal bunch populations $N_{B}^{+}=N_{B}^{-}$,

$$
B=\frac{\beta^{\star} r_{p}^{2}}{4 \pi \varepsilon f_{0} \gamma} \frac{\mathscr{L}}{\xi^{2}}=1500 \frac{\mathscr{L}\left[10^{32} \mathrm{~cm}^{-2} \mathrm{~s}^{-1}\right]}{\xi^{2}\left[10^{-6}\right]}
$$

Another reason to keep the bunch populations equal is to avoid the reduction in luminosity lifetime which inevitably occurs with unbalanced beams 5 . For example, if an initial luminosity of $10^{32} \mathrm{~cm}^{-2} \mathrm{~s}^{-1}$ is achieved with an interaction beta of 1.0 meter, colliding 1200 bunches of protons and antiprotons each containing $1.28 \times 10^{10}$ particles (case $B$ below), the final luminosity after 22 hours is $0.51 \times 10^{32} \mathrm{~cm}^{-2} \mathrm{~s}^{-1}$. This naively assumes no mechanism'for emittance blow-up or collapse, and assumes no means of particle loss other except through useful luminosity interactions. However, if the number of antiprotons per bunch is halved, but the same initial luminosity is maintained by reducing the interaction beta to 0.5 meters (case B-1 below), the final luminosity is reduced to $0.38 \times 10^{32} \mathrm{~cm}^{-2} \mathrm{~s}^{-1}$.

Under the assumptions stated above, the two main effects limiting the attainable luminosity are the antiproton production rate and the average number of events per crossing. The head-on tune shift parameter is not critical, being typically less than the maximum allowable value of 0.004 per interaction point. This value of tune shift has been experimentally determined in the 
SpDs collider, with six interaction points. ${ }^{6}$ Thus it is now possible to derive the antiproduction rate necessary for satisfactory operation at the nominal luminosity of $10^{32} \mathrm{~cm}^{-2} \mathrm{~s}^{-1}$.

Figures 4.1 and 4.2 show the dependences of the antiproton production rate and the average number of events per crossing as functions of tune shift parameter, for two different interaction beta values. Assuming $B^{*}=1.0$ meter at the interaction point and a luminosity of $10^{32} \mathrm{~cm}^{-2} \mathrm{~s}^{-1}$ (Fig. 4.1), the number of events per crossing can be kept below three only if the antiproton production rate is at least $2 \times 10^{8} \mathrm{~s}^{-1}$. For 1.5 events per crossing and the same luminosity, the production rate must be increased to $3 \times 10^{8} \mathrm{~s}^{-1}$. With a beta value of $0.5 \mathrm{~m}$ at the interaction point (Fig. 4.2), the required production rate for 1.5 events per crossing (as in the $\mathrm{pp}$ option described in (DR), and a luminosity of $10^{32} \mathrm{~cm}^{-2} \mathrm{~s}^{-1}$, is reduced to $2 \times 10^{8} \mathrm{~s}^{-1}$.

Detailed studies have been carried out for nominal machine conditions, case $A$, and for variations, cases $B$ to $D$, as shown in Table 4.4.

Table 4.4

Performance Parameters for the Four Cases Studied

\begin{tabular}{lccccccccc} 
Case & $\mathscr{L}$ & $\langle\mathrm{n}\rangle$ & $\mathrm{N}^{-}$ & $\xi$ & $B_{\mathrm{B}}^{*}$ & $N_{B}^{-}$ & $N_{B}^{+}$ & $B$ & $S_{B}$ \\
& $10^{32} \mathrm{~cm}^{-2} \mathrm{~s}^{-1}$ & & $10^{8} \mathrm{~s}$ & $10^{-3}$ & $\mathrm{~m}$ & $10^{10}$ & $10^{10}$ & & $\mathrm{~m}$ \\
\hline A & 1.0 & 1.6 & 2.0 & 0.8 & 0.5 & 0.64 & 0.64 & 2400 & 40 \\
B & 1.0 & 3.2 & 2.0 & 1.6 & 1.0 & 1.28 & 1.28 & 1200 & 80 \\
B-1 & 1.0 & 3.2 & 1.0 & 1.6 & 0.5 & 0.64 & 1.28 & 1200 & 80 \\
C & 0.5 & 3.2 & 1.0 & 1.6 & 1.0 & 1.28 & 1.28 & 600 & 160 \\
C-1 & 0.5 & 3.2 & 0.5 & 1.6 & 0.5 & 0.64 & 1.28 & 600 & 160 \\
D & 10.0 & 4.3 & 10.0 & 3.0 & 1.0 & 0.85 & 2.56 & 9600 & 10
\end{tabular}




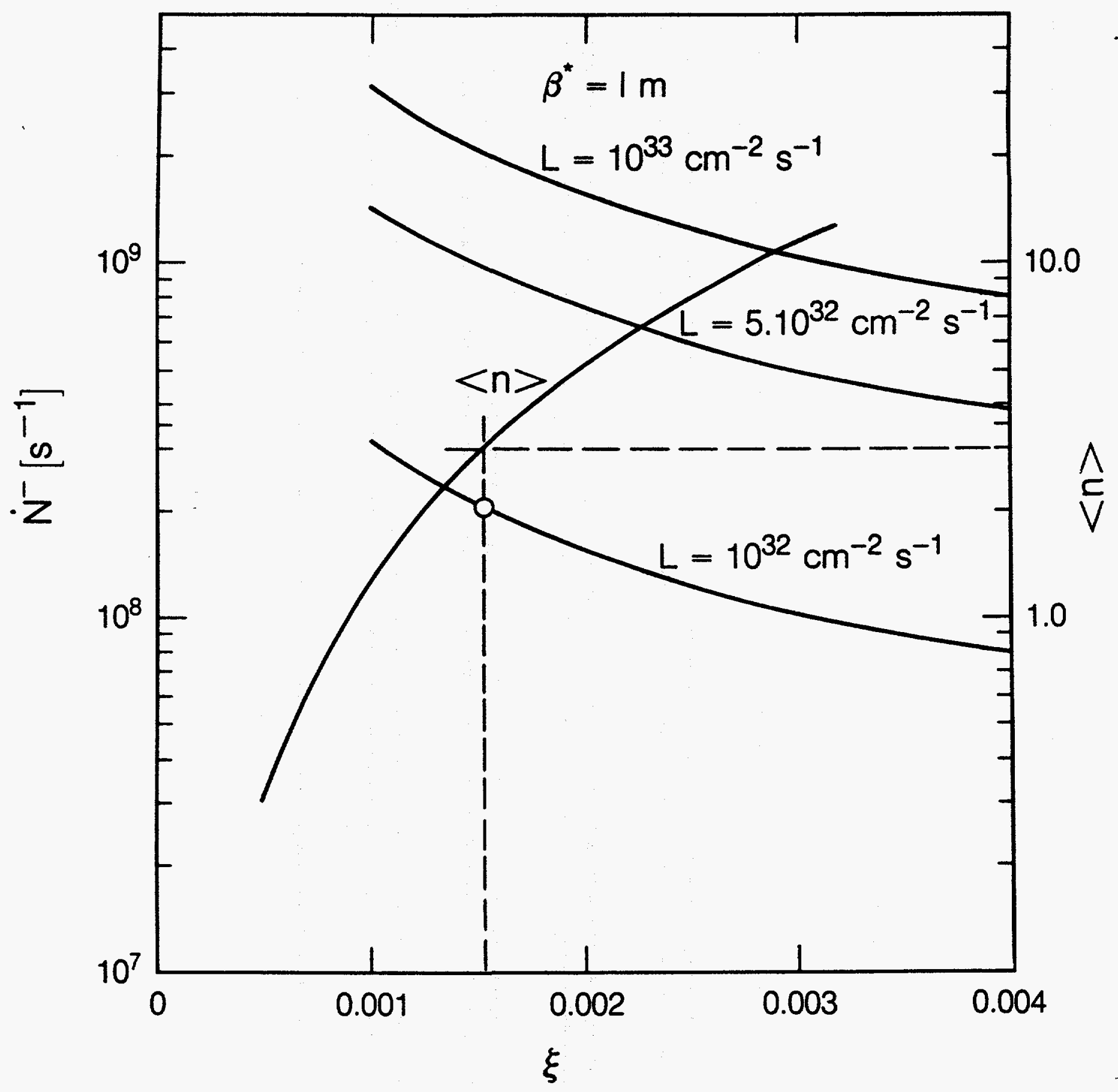

Fig. 4.1 Antiproton production rate $\left(\dot{N}^{-}\right)$and average number of events per crossing $(\langle n\rangle)$ versus tune shift parameter $(\xi)$, with interaction region beta $\left(B^{*}\right)=1.0$ meter. 


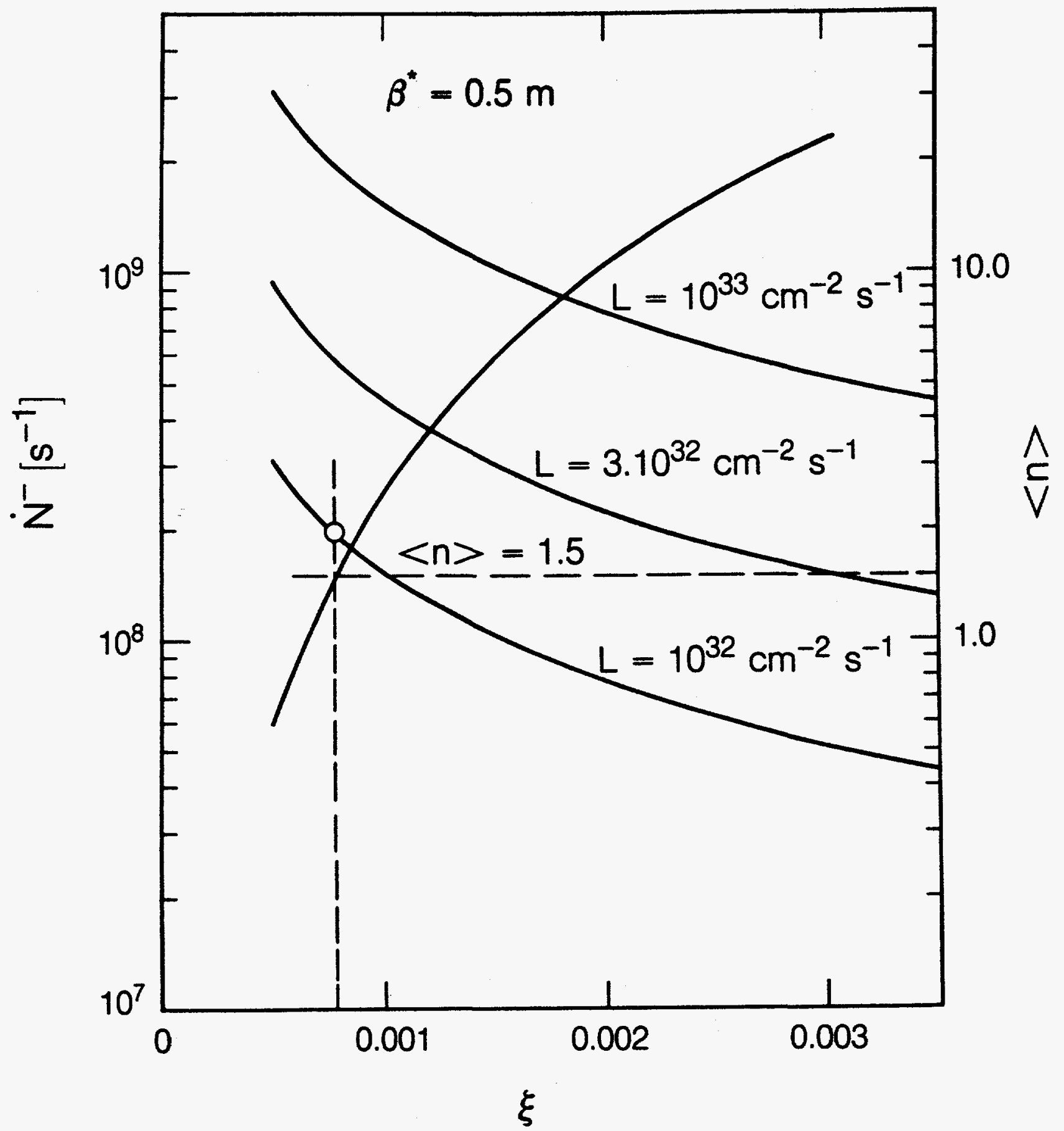

Fig. 4.2 Antiproton production rate $\left(\dot{N}^{-}\right)$and average number of events per crossing $(\langle n\rangle)$ versus tune shift parameter $(\xi)$, with interaction region beta $\left(B^{*}\right)=0.5$ meter. 
The four main cases, A to D, are distinguished by their luminosity and <n> values; with the assumption of equal currents, this is enough to specify the required antiproton source strength and the head on tune shift parameter, for a particular value of $B^{\star}$.

Comparison of cases $A$ and $B$ shows that the average number of events per interaction can be reduced from 3.2 to 1.6 , with a constant antiproton production rate of $2 \times 10^{8} \mathrm{~s}^{-1}$ and a luminosity of $10^{32} \mathrm{~cm}^{-2} \mathrm{~s}^{-1}$, by reducing $\beta^{*}$ from 1.0 meter to 0.5 meters. The total number of antiprotons is the same in both cases.

Comparison of cases $B$ and $C$ shows that the luminosity attainable is halved if the antiproton production rate is halved while the number of events per interaction and the value of $B^{\star}$ are kept fixed.

The more hypothetical fourth case, D, describes the parameters required for a luminosity of $10^{33} \mathrm{~cm}^{-2} \mathrm{~s}^{-1}$. Unequal currents are required, with roughly an order of magnitude increase in the number of bunches and in the antiproton production rate.

Cases $B-1$ and $C-1$ are variants of $B$ and $C$, showing that the luminosity can be maintained if the antiproton production rate is halved, at the expense of halving the interaction beta and accepting imbalanced currents.

The three cases with $10^{32} \mathrm{~cm}^{-2} \mathrm{~s}^{-1}$ luminosity $(A, B$, and $B-1$ ) are expected to be the same with regard to long range beam-beam interactions, since the average number of protons encountered per meter (the proton line charge) is the same in each case.

\subsubsection{Beam Separation}

The major dynamical problem of a proton-antiproton collider is the interaction of the two beams by the long range beam-beam force, resulting for 
example in an orbit distortion produced by the dipole component of the electromagnetic force. The closed orbits of the two beams circulating in the same vacuum chamber must be separated far enough to reduce this interaction to a tolerable level. Higher order multipoles are generated around the separated closed orbits by the divergence of the electromagnetic fields. Following ref. 7 , the beam-beam induced kick in the long range approximation is given, in the horizontal plane, by

$$
\begin{aligned}
\Delta x^{\prime} & =-\frac{8 \pi \xi \varepsilon}{r} \frac{x}{r^{2}}, \\
r^{2} & =x^{2}+z^{2} .
\end{aligned}
$$

Expansion in multipoles of this impulse around the separated closed orbit leads, in the horizontal and vertical planes respectively, to

$$
\begin{aligned}
& \Delta x^{\prime}=b+q x+\bar{q} z+s\left(x^{2}-z^{2}\right)+\bar{s} 2 x z+o\left(x^{3}-3 x z^{2}\right)+\bar{o}\left(3 x^{2} z+z^{3}\right)+\ldots \\
& \Delta z^{\prime}=b-q z+\bar{q} x-s 2 x z+\bar{s}\left(x^{2}-z^{2}\right)-o\left(3 x^{2} z+z^{3}\right)+\bar{o}\left(x^{3}-3 x z^{2}\right)^{2}+\ldots
\end{aligned}
$$

with the following normal and skew multipole terms:

\section{normal}

dipole

quadrupole $q=\frac{K}{d^{4}}\left(d_{x}^{2}-d_{z}^{2}\right)$

sextupole $\quad s=\frac{K}{d^{6}}\left(d_{x}^{3}-3 d x d_{z}^{2}\right)$

octupole

$$
b=\frac{k}{d^{2}} d_{x} \quad \bar{b}=\frac{k}{d^{2}} d_{z} ;
$$

skew

$$
\bar{q}=\frac{K}{d^{4}} d_{x} d_{z}
$$

$$
\bar{s}=\frac{K}{d^{6}}\left(3 d_{x}^{2} d_{z}-d_{z}^{3}\right)
$$

$$
0=\frac{k}{d^{8}}\left(d_{x}^{4}-6 d_{x}^{2} d_{z}^{2}+d_{z}^{4}\right) \quad \overline{0}=\frac{k}{d^{8}}\left(4 d_{x}^{3} d_{z}^{-4 d} d^{3} z^{3}\right) .
$$


Here, $d_{x}$ and $d_{z}$ are the total horizontal and vertical separations of the two beams respectively, and

$$
K=-\frac{8 \pi \xi \varepsilon}{r}, \quad d^{2}=d_{x}^{2}+d_{z}^{2} .
$$

\subsubsection{Separation schemes}

In principle there are three different ways to separate the two beams; these have already been discussed in refs. 7-12.

(a) Momentum separation.

(b) One-dimensional electrostatic separation (either horizontal or verti(a)1).

(c) Two-dimensional "double helix" electrostatic separation.

\section{(a) Momentum Separation}

At first glance the momentum separation scheme seems to be advantageous because no separators are needed. However, a change in momentum can only be achieved by changing either the revolution frequency $f_{0}$ or the harmonic number $K$ according to

$$
\frac{\Delta P}{P}=-\frac{1}{\alpha} \frac{\Delta f_{R F}}{f_{R F}}=-\frac{1}{\alpha}\left(\frac{\Delta f_{0}}{f_{0}}+\frac{\Delta K}{K}\right),
$$

with a the momentum compaction factor. In both cases, synchronization problems arise. Assuming a change in harmonic number of only one unit, the rf frequency must be $8.9 \mathrm{GHz}$ to produce $7.5 \mathrm{~mm}$ horizontal orbit displacement in the focusing quadrupole of a normal cell. A cell phase advance of 60 degrees has been assumed for this calculation, giving a relative momentum deviation of $1.95 \times 10^{-3}$ for a dispersion of 3.85 meters. A beam with this momentum deviation is antidamped in the horizontal plane, meaning that the advantage of synchrotron radiation damping is lost. If the revolution frequency is changed to generate 
the momentum deviation for beam separation, the revolution times are different for the two beams, so that the beam collision point in the interaction region sweeps along a length given by the bunch separation distance. Momentum separation is not considered any further in this report.

\section{(b) One-dimensional Electrostatic Separation}

It is preferable to separate in the horizontal plane, as at CESR, since vertical separation produces skew quadrupole feed-down terms in the normal chromaticity correcting sextupoles. Only a part of the ring can be filled, because the bunches must be grouped in batches in order to avoid collisions or close encounters at the crossing points of the separated orbits, as illustrated in Fig. 4.3.

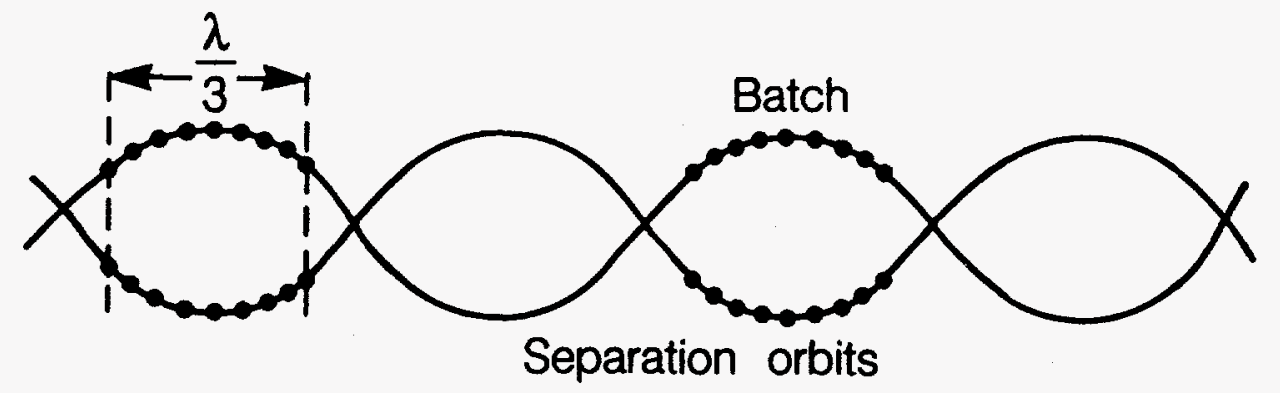

Fig. 4.3. The grouping of bunches into batches, in a one dimensional separation scheme.

This leads to a reduction in luminosity if the bunch spacing, $S_{B}$, is held constant. Another severe disadvantage is the dependence of the long range tune shift on the position of the bunch inside its batch. There is a bunchto-bunch tune spread, which either requires a larger stable area in tune 
space, or must be strongly reduced by increasing the separation amplitude. (It could also be compensated by using rf quadrupoles, which would make the machine operation even more complicated.) Furthermore, the difference between center bunch and edge bunch interactions produces slightly different closed orbits, which probably complicates the injection process. A comparison of the performance with one dimensional separation and with double helix separation has been made in 4.3 .3 .4 for case 0 , with $10^{33} \mathrm{~cm}^{-2} \mathrm{~s}^{-1}$ luminosity.

\section{(c) Double Helix Separation}

In the helical separation scheme, the beams are horizontally and vertically displaced by orthogonal electrostatic separation bumps. A great advantage of helical separation is the reduction through compensation effects of the long range beam-beam tune shift. If the separation is purely horizontal, the long range beam-beam quadrupole term is horizontal defocusing; if the separation is purely vertical, it is horizontally focusing. While in a one-dimensional separation scheme the long range beam-beam tune shifts have the same sign for each encounter, and accumulate, in a helical scheme there is considerable cancellation. The net tune shift acquired over one encounter period (one turn of the helix, assumed to be an integral number of standard cells) is not zero, but is relatively small. Its value depends on an integral over the azimuthal position of the encounters, which includes the variation of beta functions, and on the true construction of the "helix" from piecemeal line segments.

\subsubsection{Simulation of the Long Range Beam-Beam Interaction}

To explore the effects of the two beams on each other, the long range beam-beam interaction has been implemented in the particle tracking code RACETRACK $^{13}$. The interactions occur over several identical helical periods, 
as shown in Fig. 4.4 for the case of 60 degrees phase advance per cell. The separation bumps are produced by electrostatic deflecting elements at the ends and beginnings of the encounter regions. A suitable number of half cells are added to the lattice in the matching regions to generate an appropriate tune. The cell length is taken to be $200 \mathrm{~m}$.

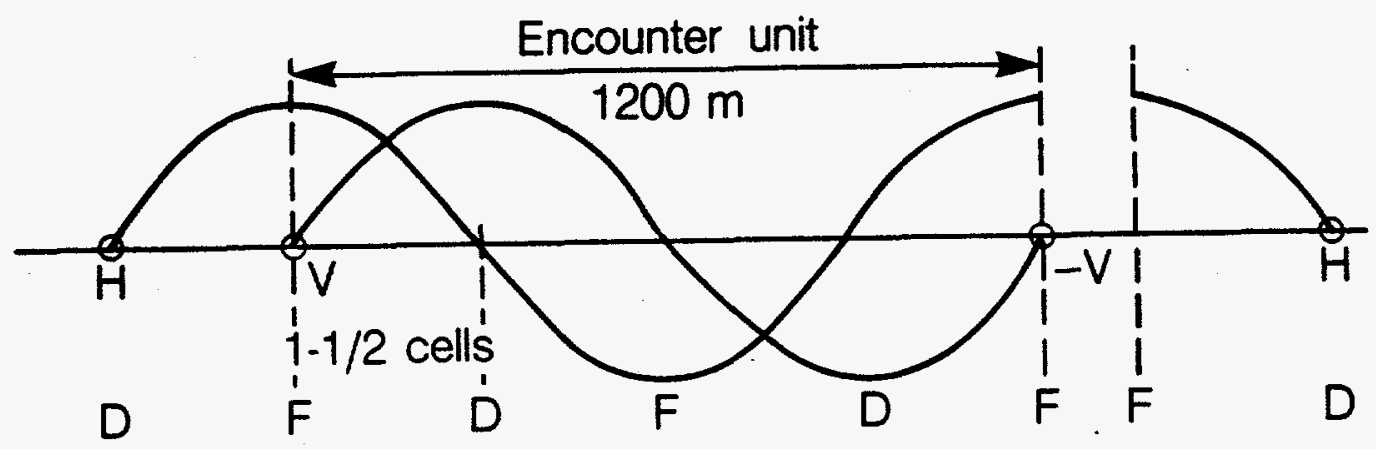

Fig. 4.4 Helical separation in an encounter region.

In lowest order the long range beam-beam effect simply distorts the separated orbits. In order to adjust these helices to be closed bumps, two additional deflection elements are included in each plane, at one end of the separation regions. Since the orbit distortions are different in both planes, the horizontal and vertical orbits lose their orthogonality and the separation distance is decreased. This is dangerous because the tune shifts and all higher order multipole effects are then increased. It is found that a beam separation over $1 / 8$ of the ring produces a phase slip which is of negligible influence. However, if the separation regions are extended beyond this range, for example in a clustered lattice, it might be necessary to include electrostatic elements as phase correctors between the ends.

If the bump is not readjusted for each beam current - for each long range interaction strength - the resulting orbit distortions add up resonantly, 
since the distortion drive has a period of $2 \pi$ betatron phase advance. In Section 4.3.3.4 a comparison of the performance with and without a compensated separation bump is given.

4.3.3.3 Beam separation for luminosities of $0.5 \times 10^{32}$ and $10^{32} \mathrm{~cm}^{-2} \mathrm{~s}^{-1}$

(cases $A, B$ and $C$ )

The quadrupole tune shift contributions due to long range beam-beam encounters oscillate in sign with double helix separation, giving, as previously mentioned, a strong compensation effect. Three different cell phase advances, 30,60 , and 72 degrees, have been investigated to explore the dependence of this compensation on the separation region optics. Table 4.4 shows that the reduction in luminosity from case $B\left(10^{32} \mathrm{~cm}^{-2} \mathrm{~s}^{-1}\right)$ to case $C\left(0.5 \times 10^{32} \mathrm{~cm}^{-2} \mathrm{~s}^{-1}\right)$ is solely due to the reduction of the number of bunches from 1200 to 600 : the tune shift parameter is the same in both cases. This implies that the beam-beam effects are expected to be stronger in case $B$, since they depend only on the tune shift parameter times the number of encounters per meter, if the discrete nature of the encounters can be ignored.

Figure 4.5 plots the vertical tune shift due to 1200 encounters per turn as a function of the maximum total helical separation, corresponding to the 600 bunches of case $C$, for the three phase advances. The separation bumps are always adjusted to give the same maximum beam separation in both planes, so the horizontal tune shifts are very similar to the vertical tune shifts. Figure 4.6 plots the variation of vertical tune shift with separation for 2400 encounters, corresponding to case $B$, and shows stronger behavior for all three phase advances, as expected. Figure 4.7 shows that the distortion of the beta functions is moderate for reasonable separation amplitudes. 


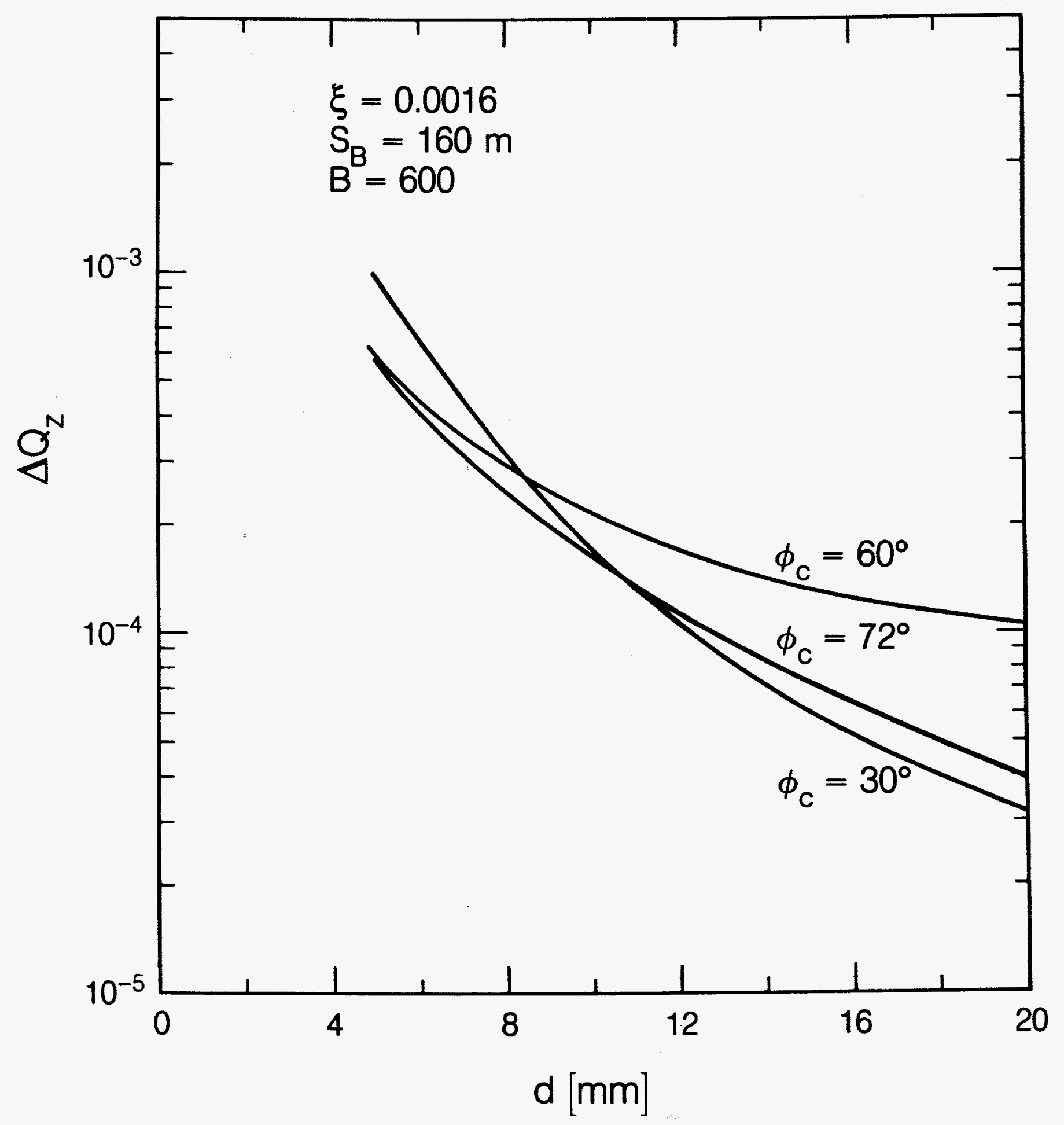

Fig. 4.5 Variation of vertical tune with total helical separation, in case $C$. 


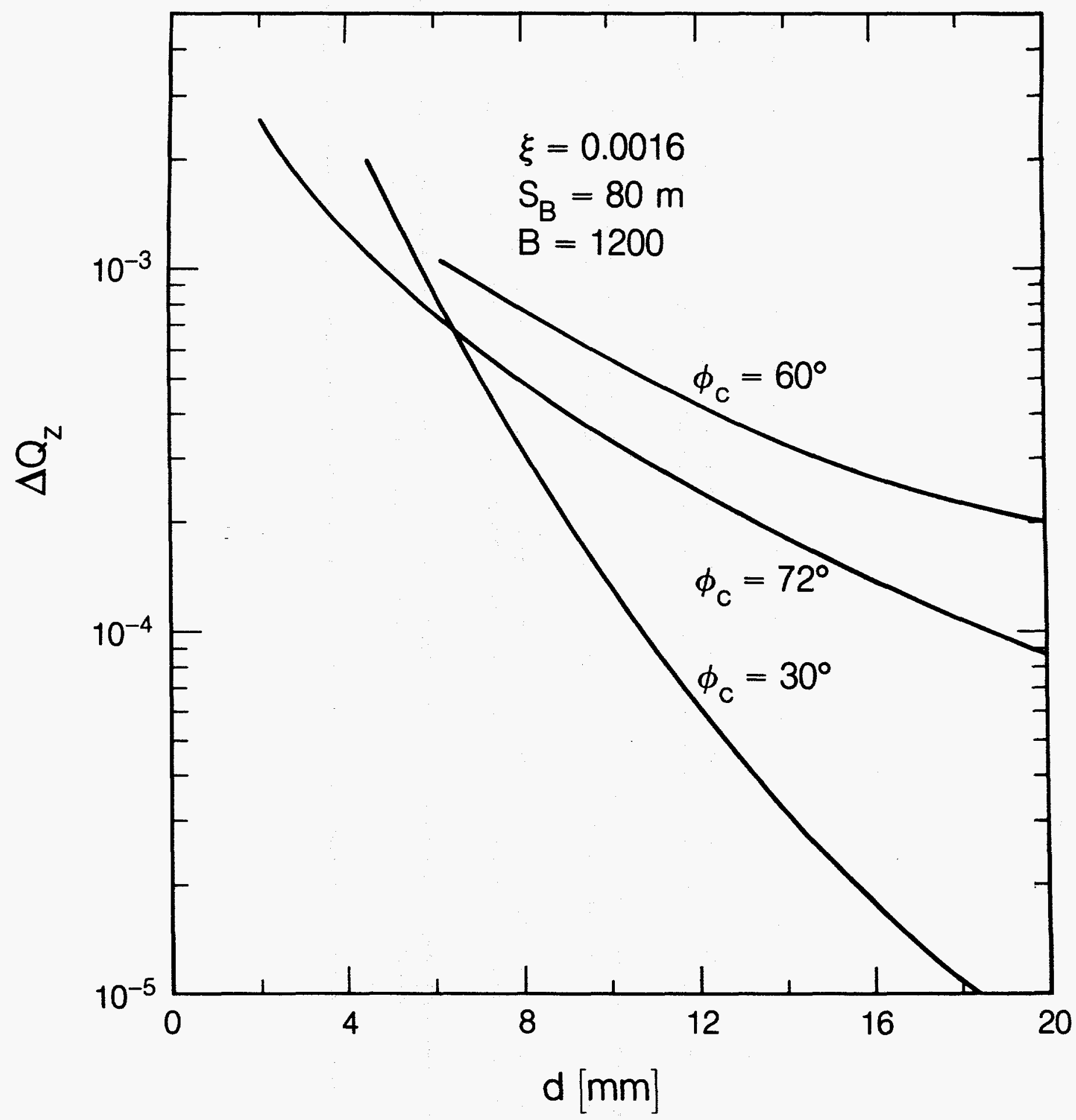

Fig. 4.6 Variation of vertical tune with total helical separation, in case B. 


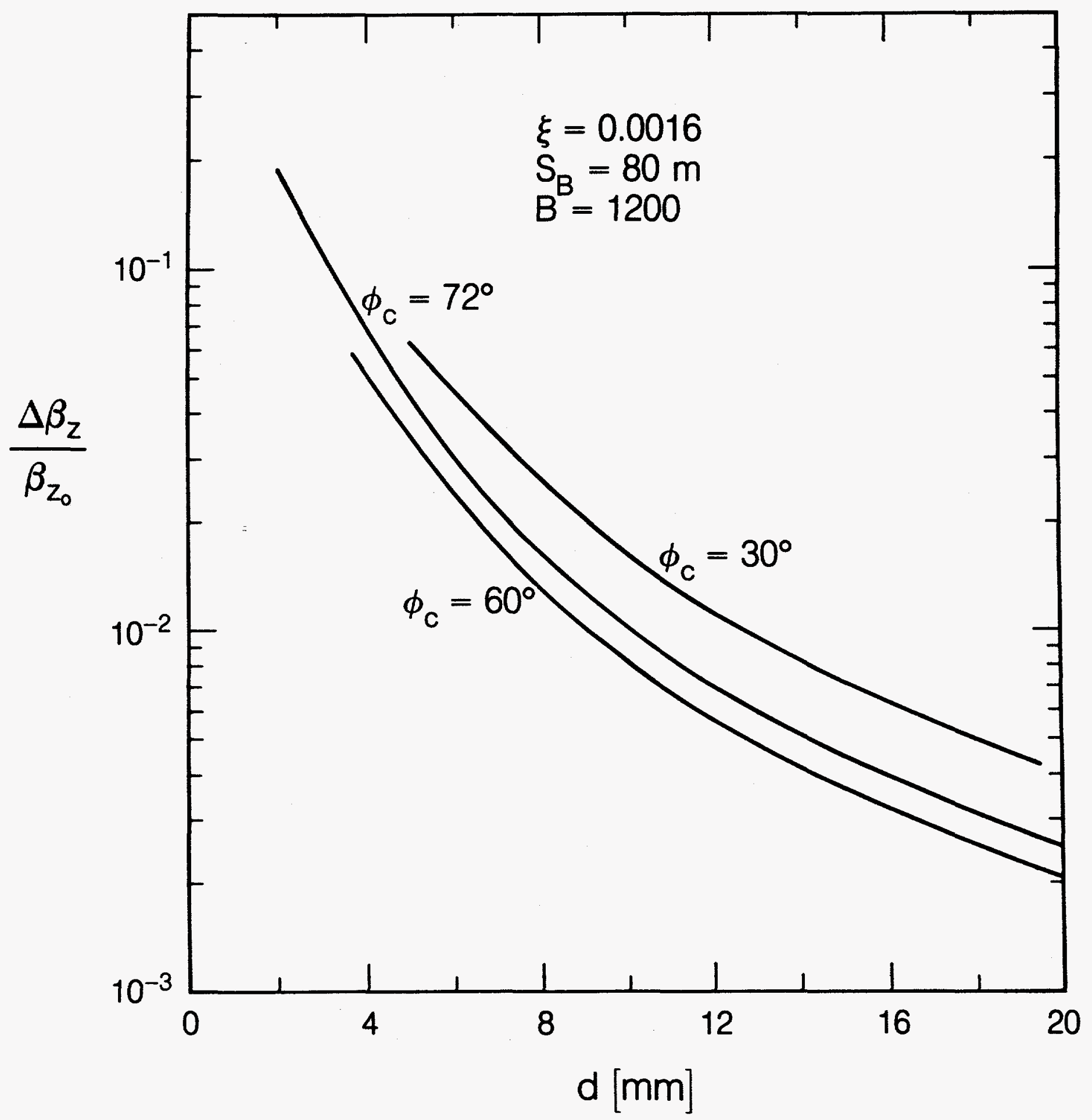

Fig. 4.7 Variation of vertical beta with total beam separation, in case B. 
The single phase advance of 72 degrees per cell, rather than the 60 degree value assumed in the CDR, is chosen as an optimal case for further study based on the somewhat better performance in tune shift shown in Figs. 4.6 and 4.7 . Although 30 degree cells appear superficially attractive, they are rejected because the peak beta in a regular cell is increased to 504 meters (assuming thin lenses and 100 meter half cell length). This would lead to an even larger increase in the required magnet bore size. The change from 60 to 72 degrees decreases the peak beta in a regular cell slightly, from 346 to 334 meters.

The chromaticity produced by the long range beam-beam sextupole field is shown in Fig. 4.8 as a function of beam separation. It varies roughiy as the inverse cube of the separation amplitude. Because the chromaticities are opposite in sign for the two closed orbits, it is necessary to compensate the overall effect by alternating the polarity of successive helices. An ensemble of four particles is tracked over 100 revolutions, so defining the dynamic aperture, in order to explore the influence of the nonlinear beam-beam multipoles on particle dynamics. Figure 4.9 shows the variation of the maximum stable amplitude with the beam separation amplitude. The results indicate a much stronger impact on the selection of the separation amplitude than the tune shift. The variations plotted for cases A and B show essentially identical behavior, because the current per bunch times the number of encounters is the same in both cases.

The dynamic apertures plotted in Fig. 4.9 are solely due to nonlinear beambeam interactions. In the absence of beam-beam interactions, and with no orbit separation, the dynamic aperture of a proton-proton SSC is $9.1 \pm 1.5 \mathrm{milli-}$ meters without the sorting of 4.0 centimeter bore dipoles, and $12.0 \pm 1.5$ millimeters with dipole sorting, according to the CDR. A nominal separation value must therefore be chosen with a beam-beam dynamic aperture substantially 


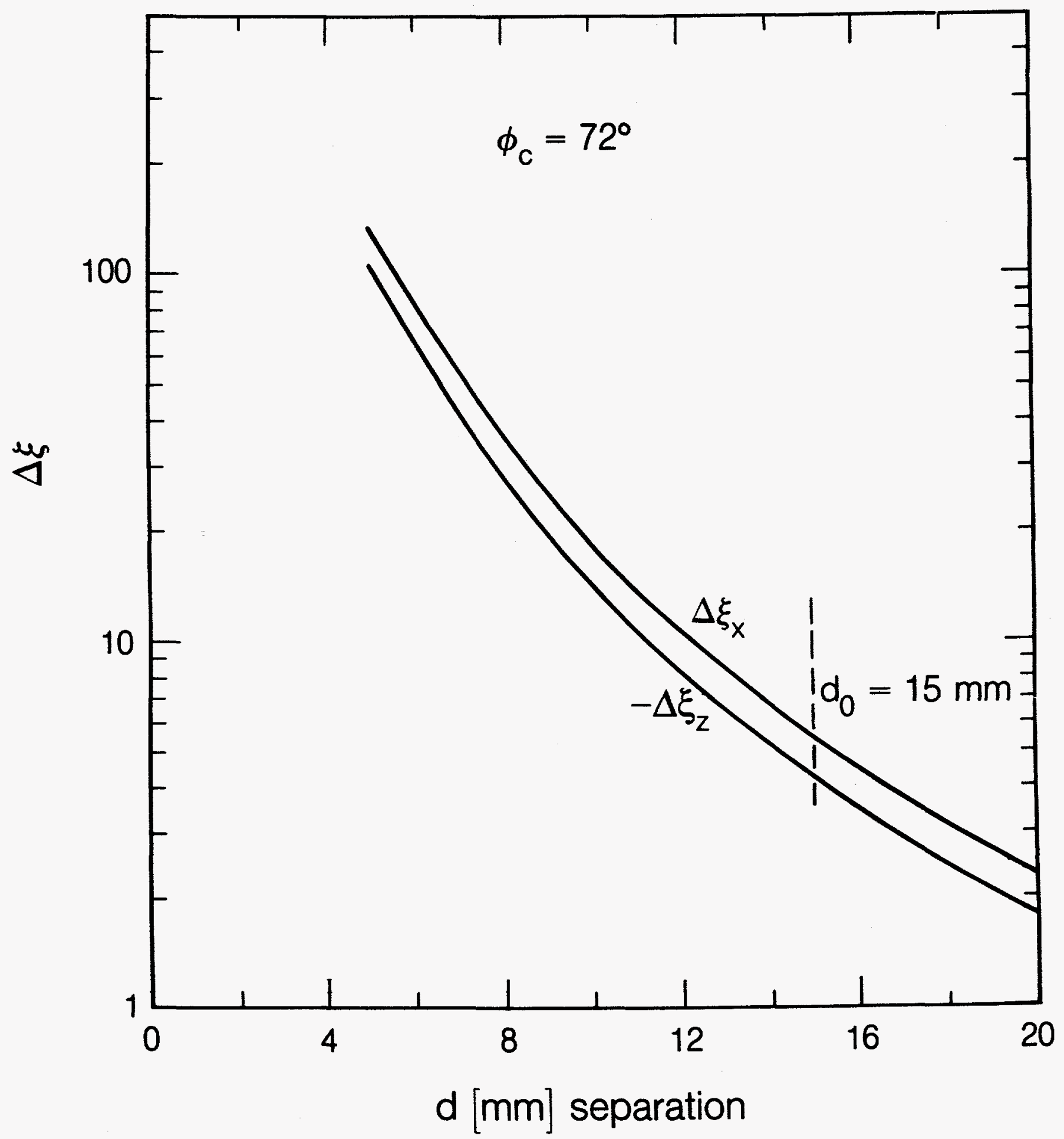

Fig. 4.8 Variation of chromaticity production due to the large range beambeam interactions, versus total separations, in case $B$. 


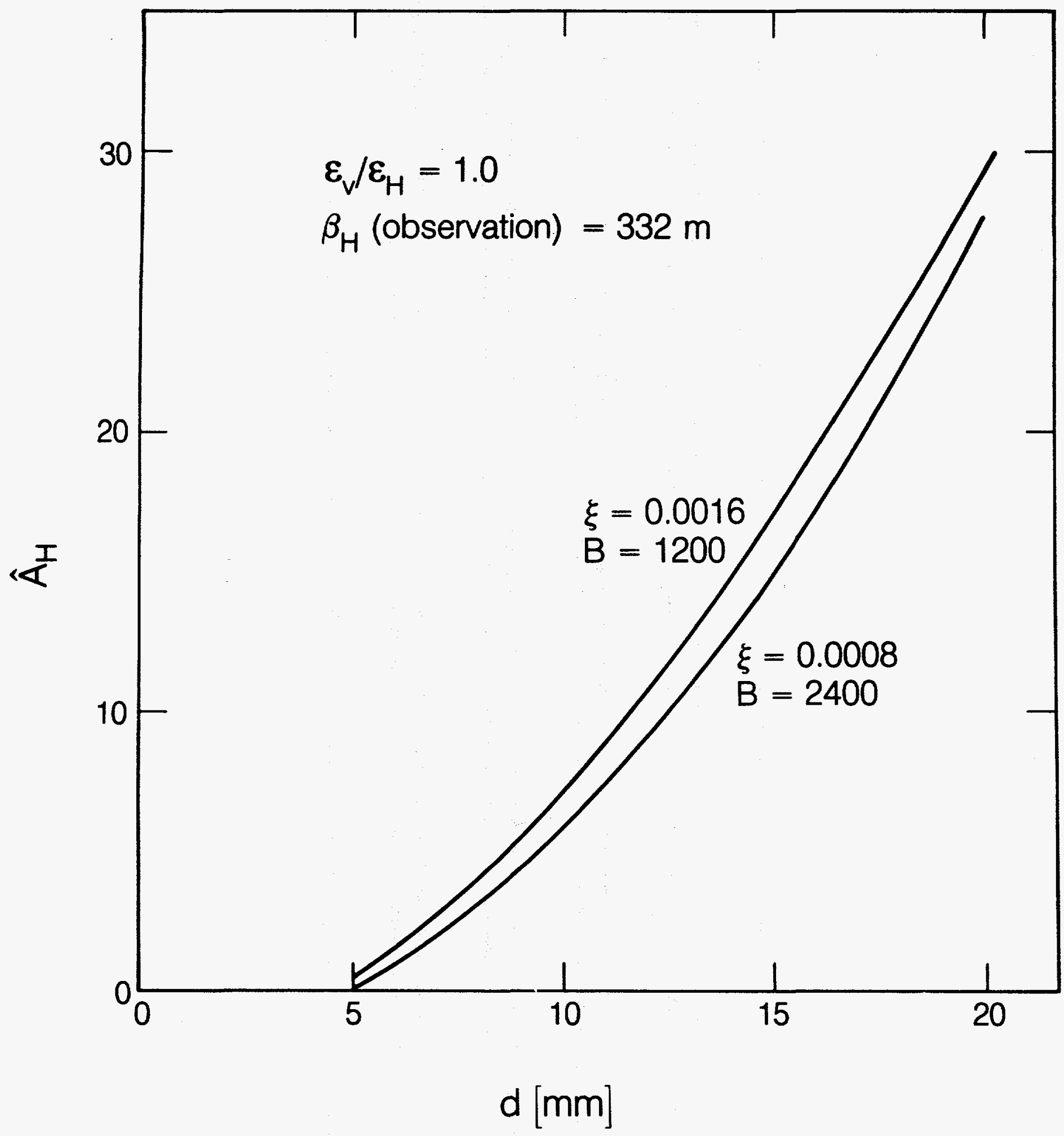

Fig. 4.9 Maximum stable amplitude, $A_{H}(\mathrm{~mm})$, versus total separation amplitude, due to long range beam-beam interactions only. 
larger than these values, in order that the net dynamic aperture is not significantly decreased when both magnetic and beam-beam sources of nonlinearity are included. The nominal maximum helical separation amplitude is thus chosen to be 15.0 millimeters, indicating a beam-beam dynamic aperture of 14.8 millimeters for case $A$, and 17.0 millimeters for case B.

The variation of tune shift with amplitude for $12.5 \mathrm{~mm}$ beam separation, found by Fourier analyzing the particle motion over 1000 turns, is illustrated in Fig. 4.10. The tune shift is less than $5 \times 10^{-4}$ for a betatron amplitude which corresponds to 4 standard deviations, and is therefore insignificant. It should be noted that the leading term producing tune shift with amplitude, the octupole, decreases with the fourth power of the beam separation.

When magnetic non-linearities are taken into account, two other effects are introduced, apart from the obvious further reduction of the dynamic aperture, which influence separated orbit operation. First, the dipole feed-down of the magnet multipole errors disturbs the separation bump. This is controlled by tuning the correctable separation elements which already have been invoked at one end of the separation region. Unfortunately, the feed-down effects are different for the two helical orbits with opposite separation amplitudes. To overcome this problem, electrostatic dipole correction must be augmented by magnetic dipole correction. Second, the quadrupole feed-down leads to a tune shift which again is different in magnitude for opposite beam separations.

Figure 4.11 shows the feed-down effect on the tune as a function of separation amplitude, superscripts + and - indicating beam separations of opposite sign. The same relationship is plotted in Fig. 4.12 for a different set of random multipoles, indicating a strong dependence of the feed-down on the random distribution. The size of these tune shifts makes compensation necessary. 


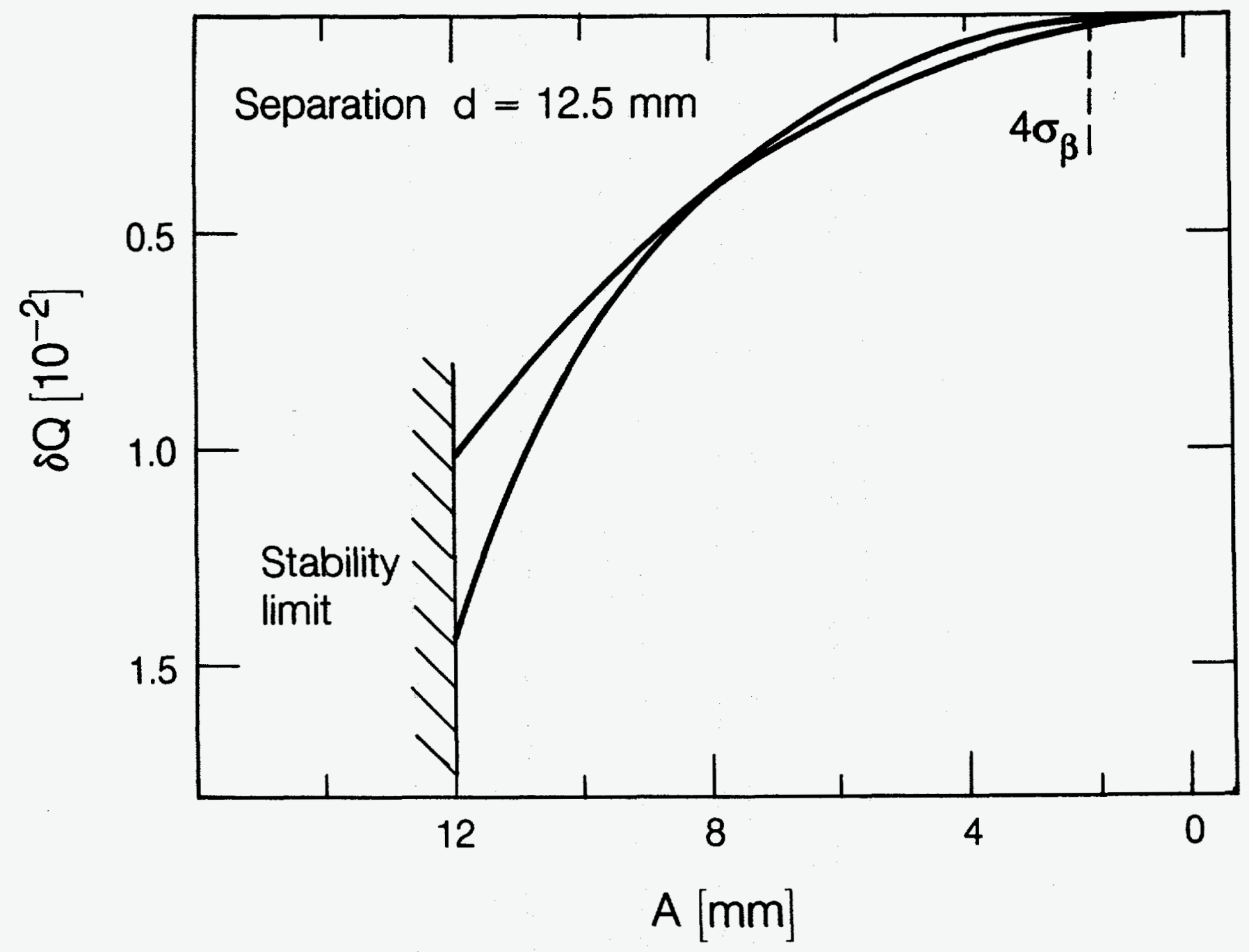

Fig. 4.10 Variation of tune with amplitude due to long range beam-beam interactions. 


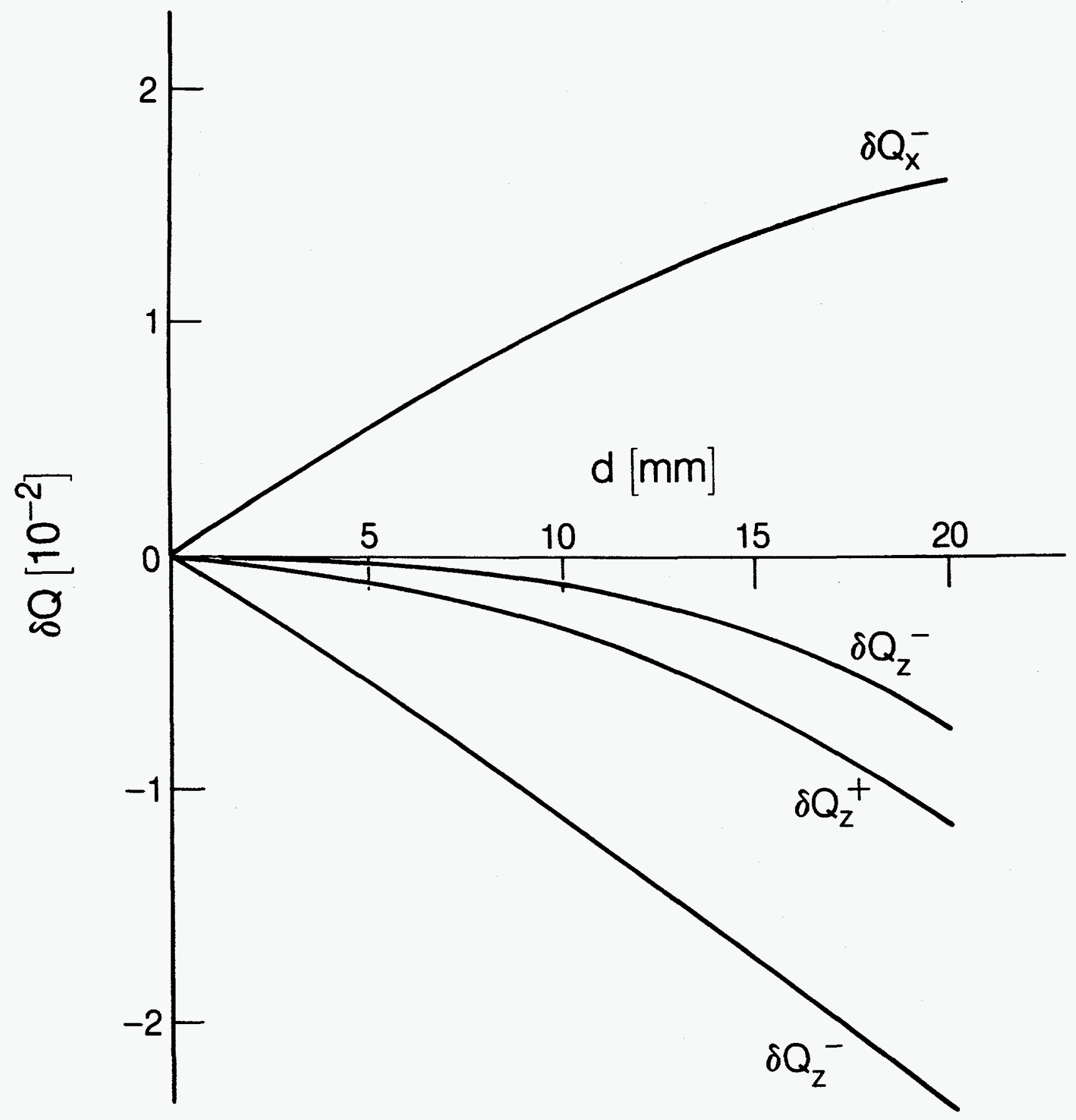

Fig. 4.11 Tune shifts due to the feed-down of random multipoles versus the total separation amplitude, first seed. 


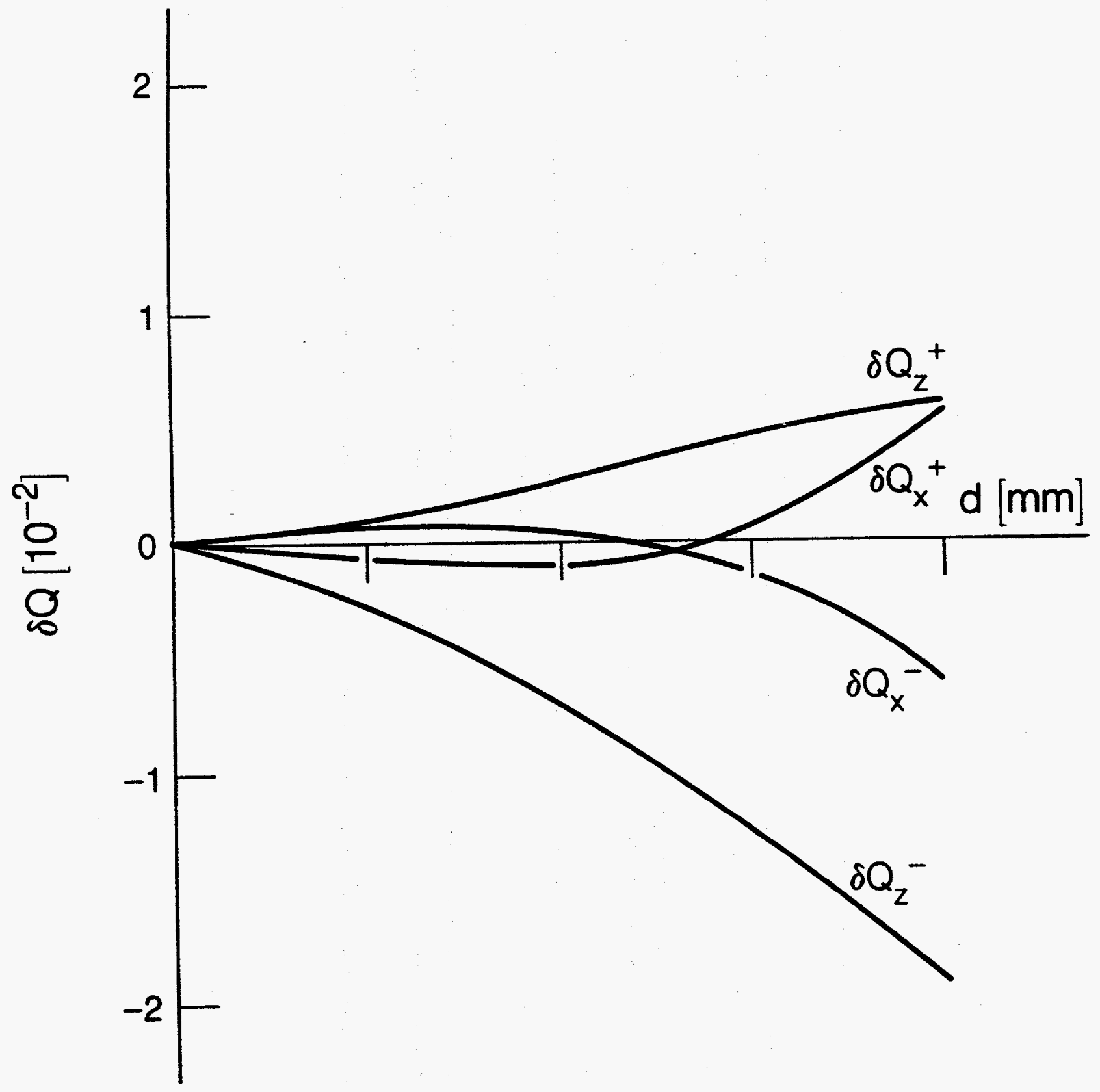

Fig. 4.12 Tune shifts due to the feed-down of random multipoles versus the total separation amplitude, second seed. 
No magnet sorting has been taken into account. The variation of the beta function produced by the feed-down, for the same random errors as for Fig. 4.11, is shown in Fig. 4.13. It is quite moderate and needs no special compensation.

Multipole feed-down has so far been considered for a constant magnet aperture of $40 \mathrm{~mm}$. However, the bore size of the magnets must be increased to compensate for the aperture loss due to beam separation, and the magnetic errors will correspondingly decrease, leading to a reduction in the strength of feed-down effects. The multipole strengths are assumed to scale according to

$$
a_{n}, b_{n} \sim(\text { bore size })^{-n-\frac{1}{2}}
$$

as proposed in ref. 14. Figure 4.14 shows the variation of the tune shifts due to the quadrupole feed-down as a function of magnet bore size. The curves converge to the values given by the chromaticity correcting sextupoles alone, since their strength does not change with the magnet size.

Quadrupoles are used to compensate for the common part of the tune shift of the two separation orbits. The larger remaining differential tune shift is adjusted by using multipoles to generate feed-down quadrupole terms which depend on the sign of the separation amplitude. Sextupoles are the lowest order able elements to do this. Figure 4.15 shows an arrangement of sextupole correctors to compensate for the feed-down effect, in the case of 72 degrees phase advance per cell.

The results presented in Fig. 4.16 illustrate the performance of this scheme; the maximum stable amplitude found by tracking is plotted as a function of the total beam separation amplitude, when a tune shift of $\Delta Q=0.015$ is corrected in both planes simultaneously. Random magnet errors are not present, so the only non-linearities come from the long range beam-beam 


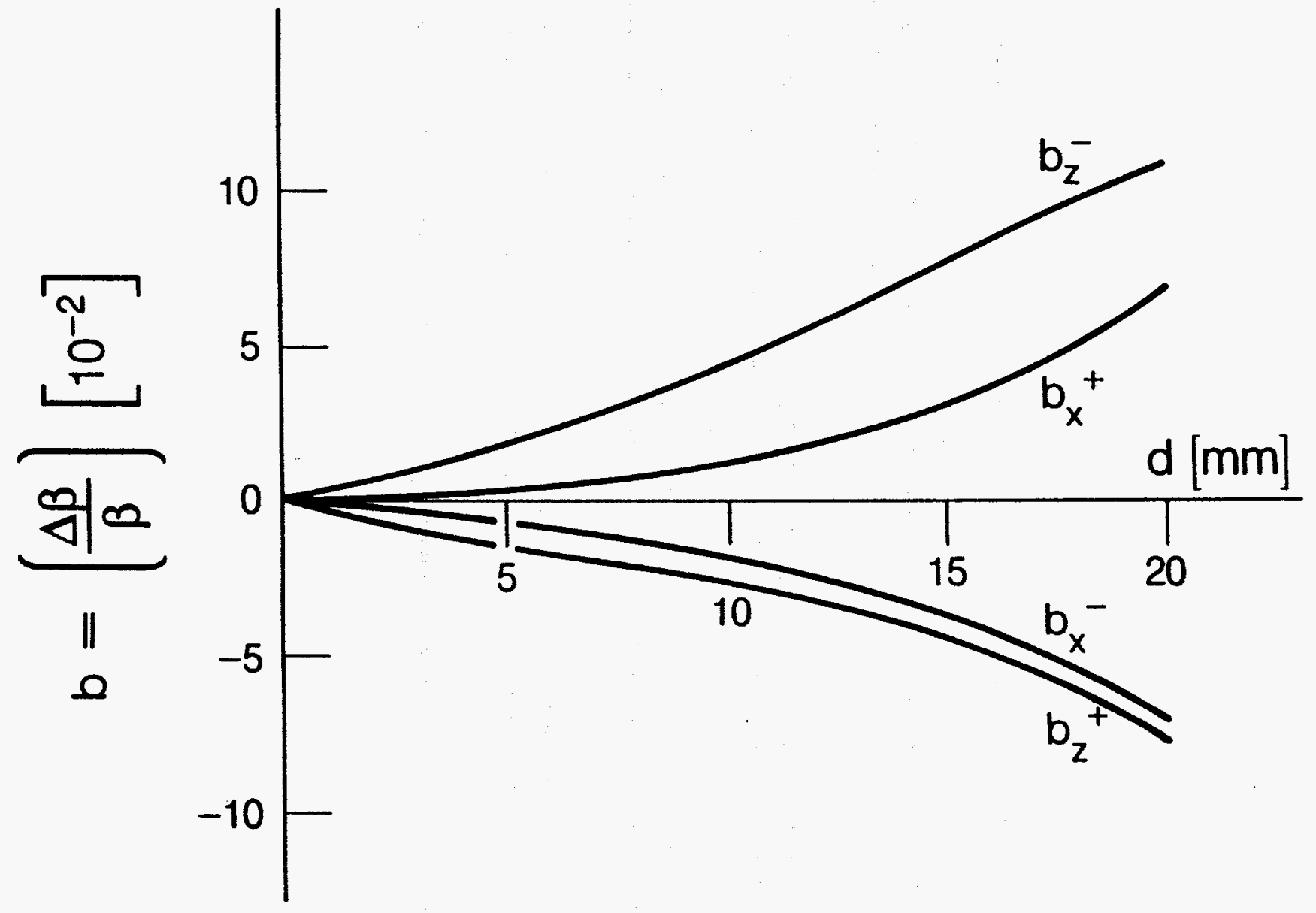

Fig. 4.13 Beta variation due to random multipole feed-down versus total separation amplitude. 


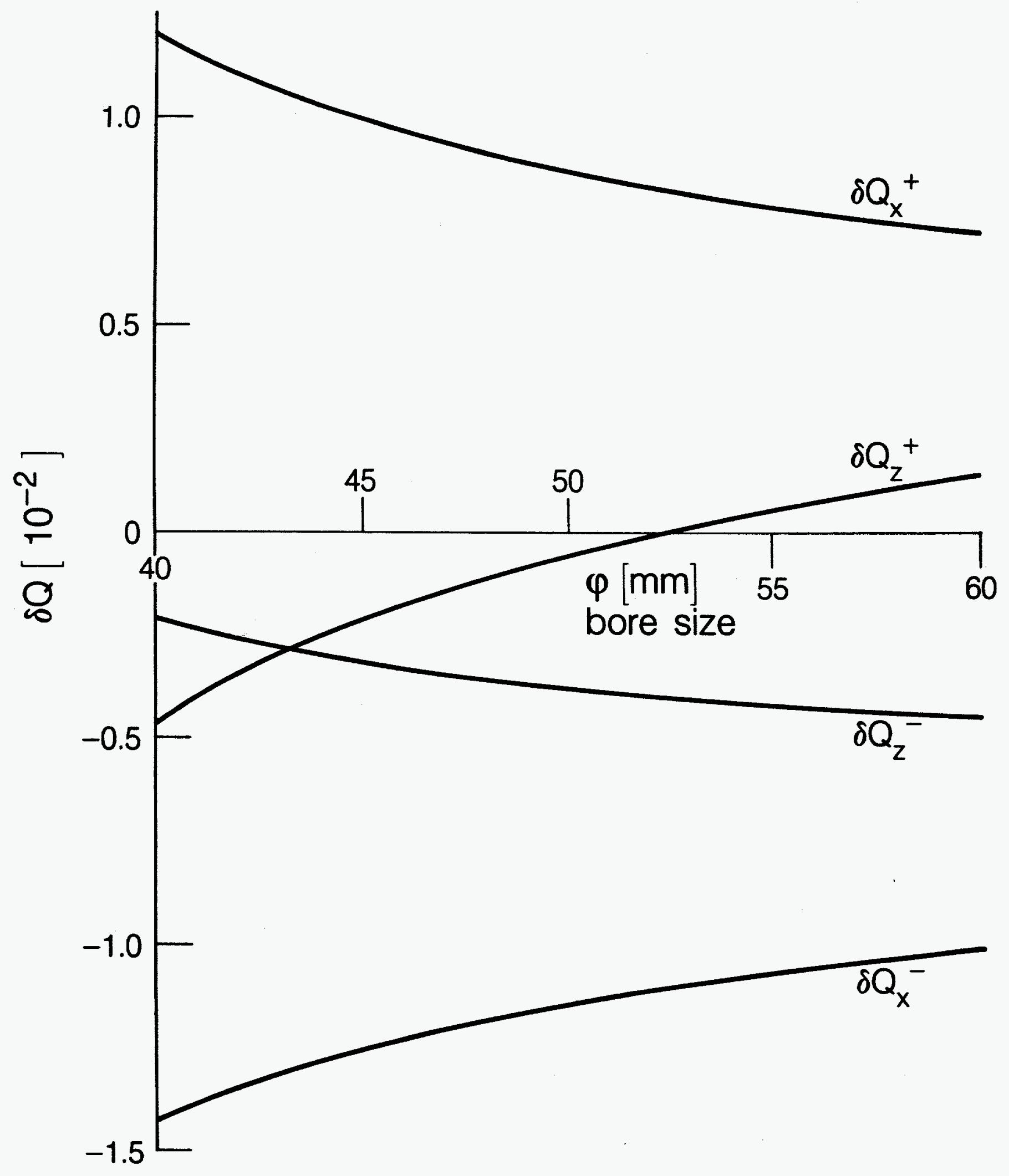

Fig. 4.14 Tune shift due to random multipole feed-down versus magnet bore size, $\mathrm{mm}$. 


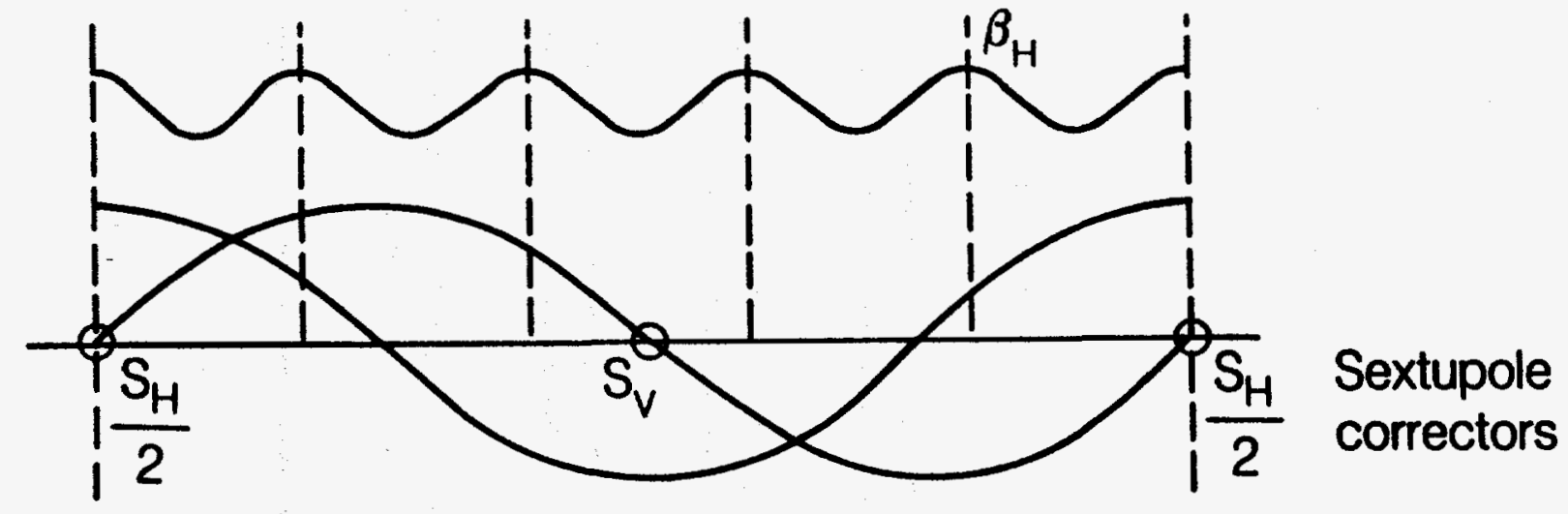

Fig. 4.15 A sextupole corrector scheme to compensate for the feed-down effect. 


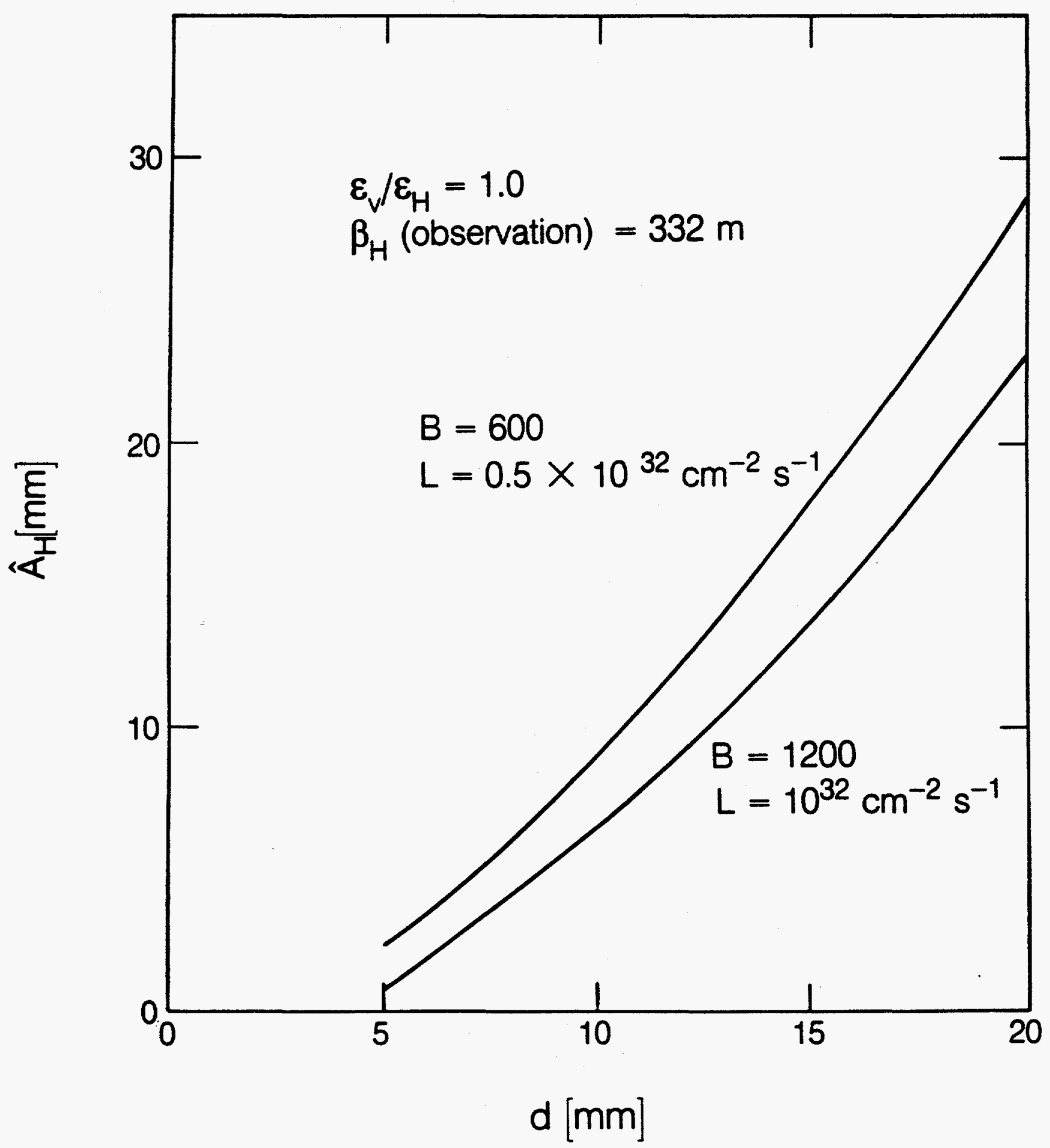

Fig. 4.16 Maximum stable amplitude versus beam separation, cases $A$ and $C$, due to long range beam-beam interactions on $1 y$, but with tune shift adjustment by corrector sextupoles. 
interactions, and the sextupoles in the correction scheme. Comparison with Fig. 4.9 shows that the sextupole correctors lead to a further reduction in dynamic aperture. For example, the dynamic aperture for case B is decreased from 17.0 to 14.8 millimeters.

Figure 4.17 shows the maximum stable amplitude as a function of beam separation in a realistic machine. Chromaticity correcting sextupoles (enhanced by a factor of three to simulate the chromaticity contribution of the low beta insertions) and random multipoles up to 20-pole, both normal and skew, are included. The bore size is increased by the amount of the separation, and the tunes are readjusted by sextupole correctors to the values of the undistorted machine. The dotted line indicates the maximum stable amplitude when it is limited solely by the chromaticity correcting sextupoles and the random multipoles, for a $4 \mathrm{~cm}$ bore. At small separation values the beam-beam interaction dominates. At large beam separations the feed-down effect increases and the sextupole correctors dominate, lowering the dynamic aperture.

\subsubsection{Beam Separation for a Luminosity of $10^{33} \mathrm{~cm}^{-2} \mathrm{~s}^{-1}$ (case D)}

The problems arising from beam separation for even larger currents are discussed in this section, under the assumption that developments in antiproton production will exceed the present state of the art, so that a production rate of $10^{9}$ antiprotons per second will be possible. A luminosity of $\mathscr{L}=$ $10^{33} \mathrm{~cm}^{-2} \mathrm{~s}^{-1}$ can be achieved for the machine conditions given by the set of parameters shown in Table 4.4 for case $D$. The number of bunches is increased to 19200 to reduce the average number of events per crossing to 4.3 , and this leads to unequal bunch populations. It is instructive to compare a one-dimensional separation scheme and a helical separation scheme for such a machine, with 60 degrees phase advance per cell. 


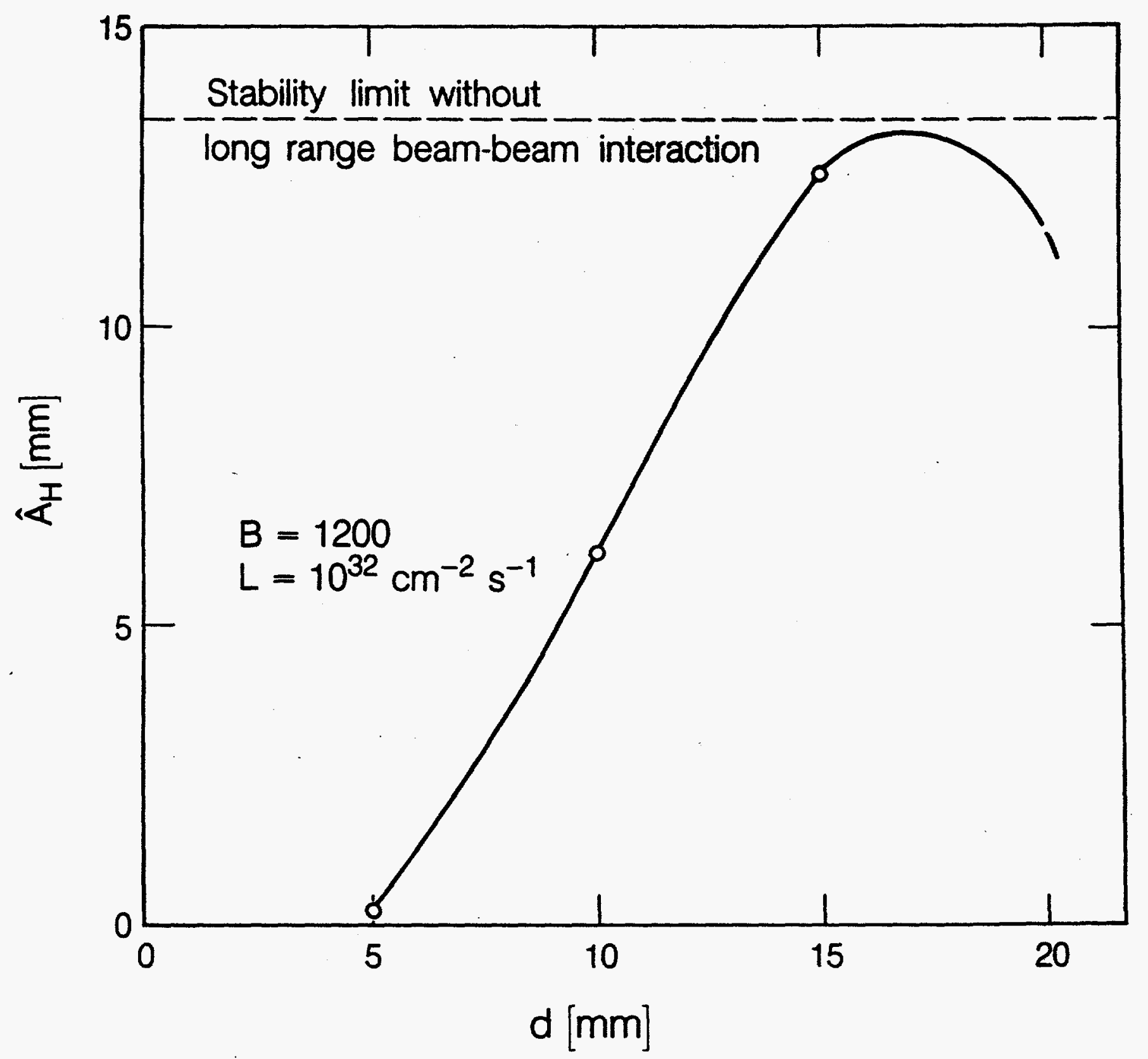

Fig. 4.17 Maximum stable amplitude versus beam sepsration in a realistic machine with chromaticity sextupoles, long range beam-beam interactions, and random multipoles, in case $A$. 
Figure 4.18 shows the beam-beam tune shift for two different bunches in a one dimensional scheme, one in the middle and one at the edge of a batch. The dashed curve shows for comparison the effect of a helical separation with three times the luminosity. In no case is the separation readjusted to compensate for the dipole distortion produced by tiie beam-beam effect. The separation must be increased above 17 millimeters in order to reduce tune shift below 0.003

The variation of horizontal tune with separation amplitude is shown in Fig. 4.19, where now the helical orbit separation is reclosed for each amplitude by adjusting the separator strengths. Finally, Fig. 4.20 shows the maximum stable amplitude as a function of beam separation, for two different tunes. This indicates that a total separation of at least $50 \mathrm{~mm}$ is necessary to increase the stable amplitude beyond the limit given for normal sextupoles and random multipoles.

\subsubsection{Separation Induced Dispersion}

The displacement caused by a single helix, at a reference point with a betatron phase 0 , is in general

$$
x=\beta^{1 / 2} / 2 \sin (\pi Q)\left[\beta_{1}^{1 / 2} \Delta x^{\prime}{ }_{1} \cos \left(\left|\varnothing-\sigma_{1}\right|-\pi Q\right)+B_{2}^{1 / 2} \Delta x^{\prime}{ }_{2} \cos \left(\left|\varnothing-\phi_{2}\right|-\pi Q\right)\right] \text {. }
$$

where $\Delta x^{\prime}{ }_{1}$ and $\Delta x^{\prime}{ }_{2}$ are the electrostatic kicks at the beginning and end of the helix. Note that, unless specifically stated otherwise, the displacements (and the dispersions) in all the discussions below may be in either plane. If the reference point is outside the helix, say with $\sigma_{0}<\phi_{2}$, at a place with a peak beta $B_{\max }$ in a F000 cell, then the displacement becomes

$$
x=h_{x} / 2 \sin (\pi Q)\left[\cos \left(\theta_{7}-\theta-\pi Q\right)-\cos \left(\theta_{2}-\phi-\pi Q\right)\right],
$$




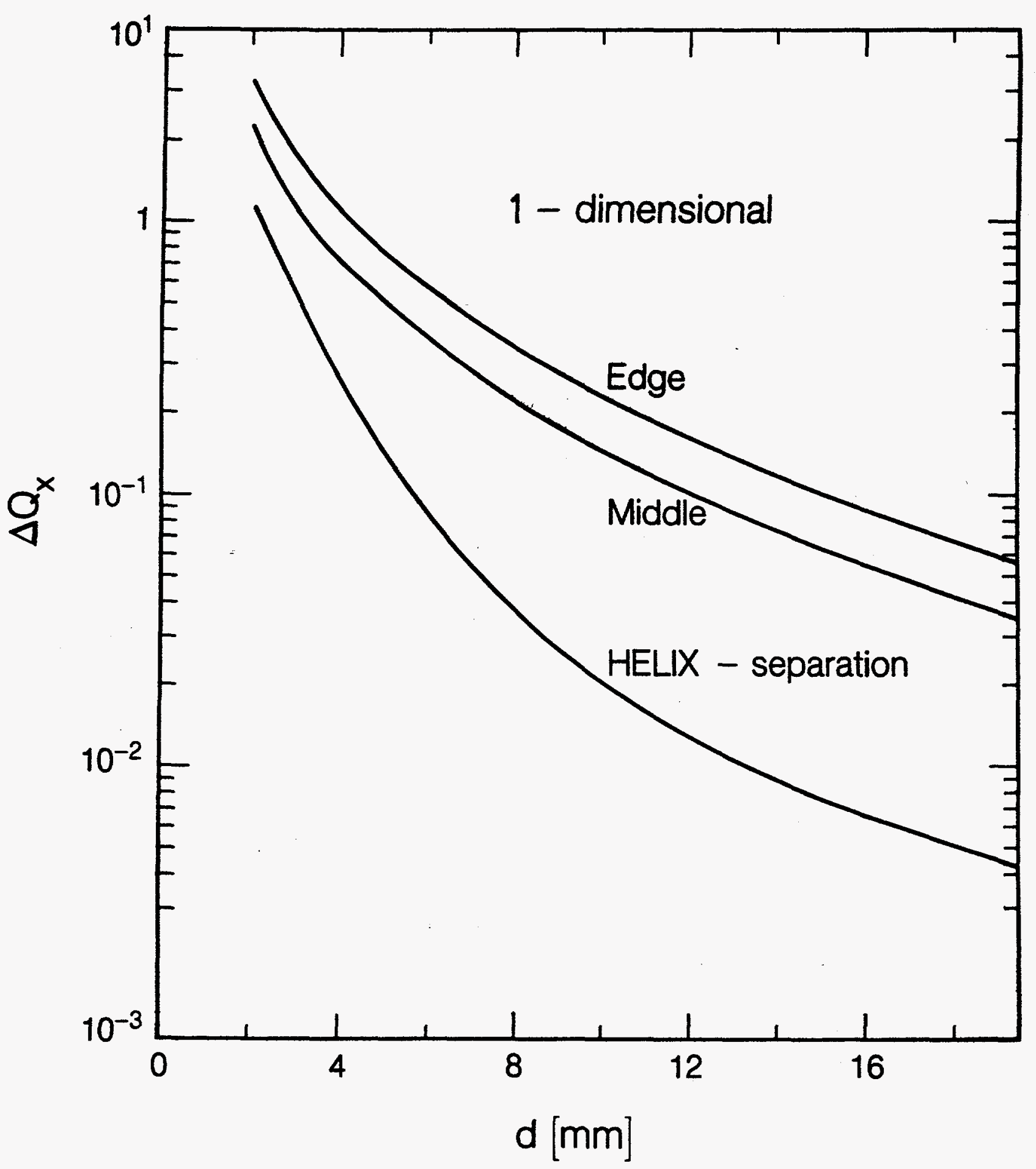

Fig. 4.18 The horizontal tune shift versus separation amplitude for one dimensional separation, compared with helical separation. 


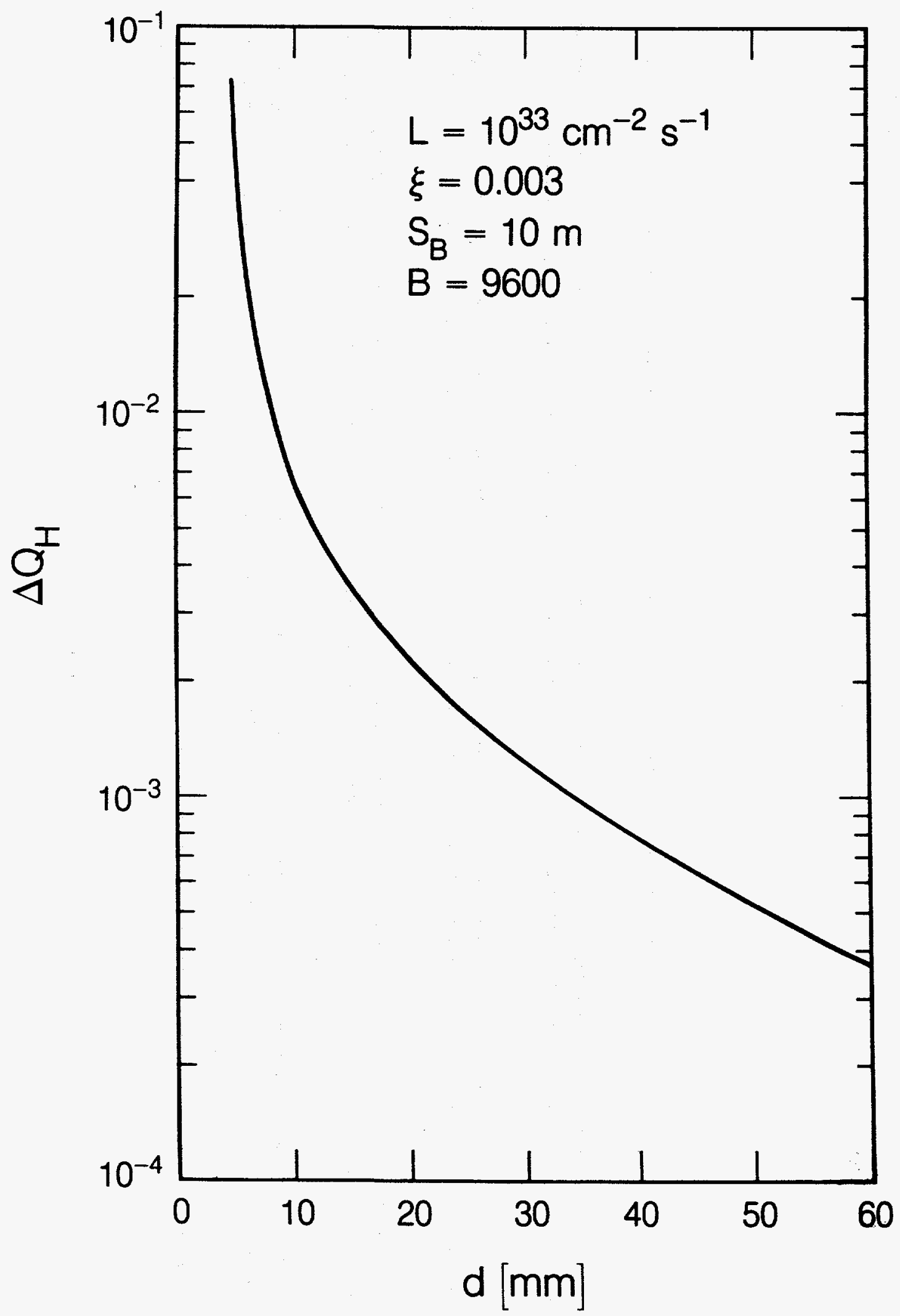

Fig. 4.19 Horizontal tune shift versus total separation amplitude in case $D$, with $\mathscr{L}=10^{33} \mathrm{~cm}^{-2} \mathrm{~s}^{-1}$. 


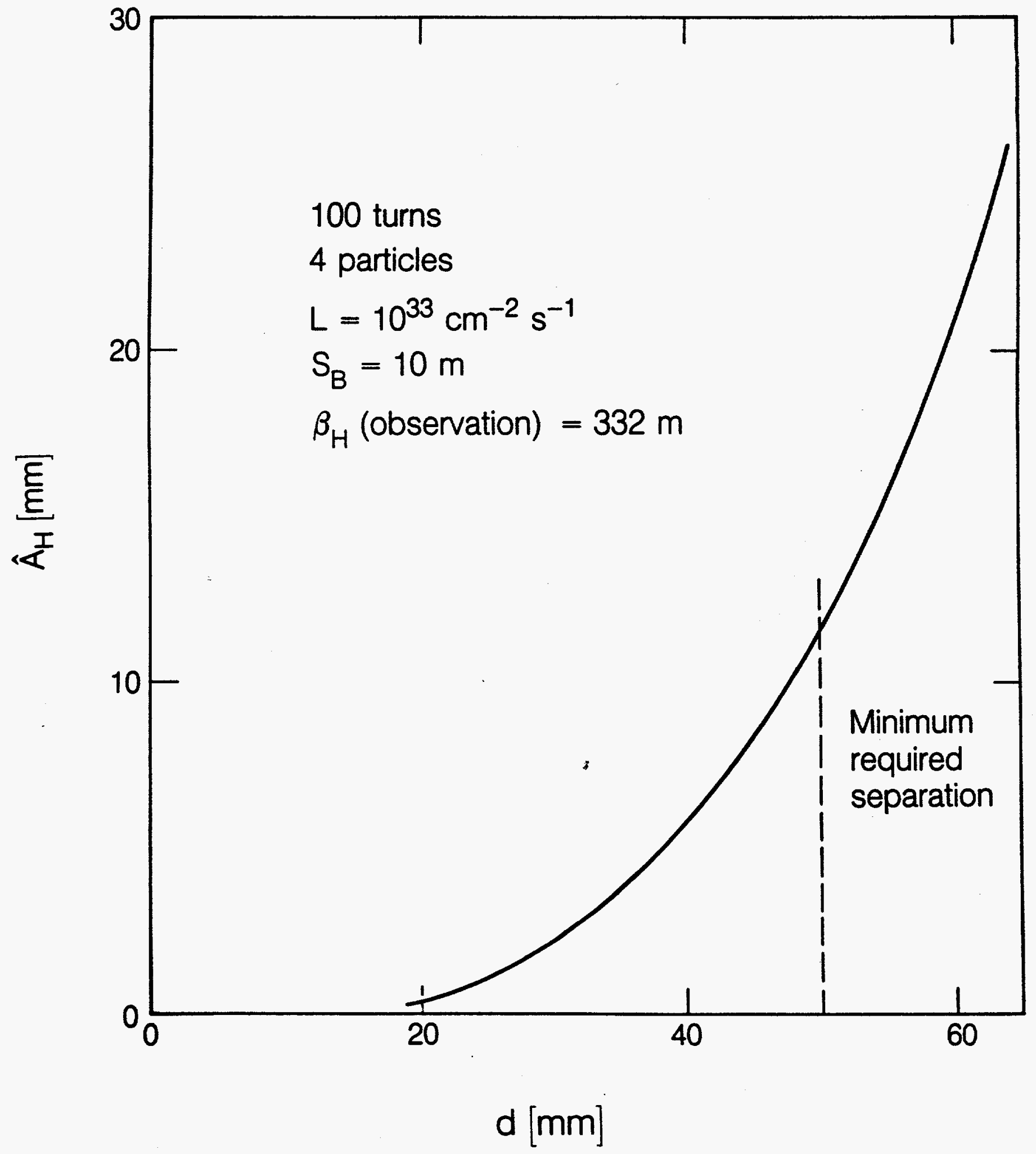

Fig. 4.20 Maximum stable amplitude versus total beam separation in case 0 , with $\mathscr{L}=10^{33} \mathrm{~cm}^{-2} \mathrm{~s}^{-1}$. 
where $h_{x}$ is the amplitude of the helix. To be definite we assume that a separation helix (or pretzel) has an integral number $a_{h}$ of wavelengths, so that

$$
\sigma_{2}=\sigma_{1}+2 \pi Q_{h} \text {, }
$$

making the term in square brackets on the right hand side of equation (2) zero and closing the helix for on-energy particles.

The dispersion perturbation caused by the helix is found by differentiating equation (1) with respect to $\delta=\Delta E / E$, the relative energy offset, so that outside the helix the dispersion is

$$
n_{x}=d x / d \delta=h_{x} / 2 \sin (\pi Q) \sin \left(\sigma_{1}-\phi-\pi Q\right) d\left(\phi_{2}-\sigma_{7}\right) / d \delta \text {. }
$$

This shows that the helix is not perfectly closed for off-energy particles, if the phase advance across it varies with energy. (In differentiating the term in square brackets it is assumed that the reference point phase is held constant $(d ø / d \delta=0)$, and that the chromaticity has been corrected $(d Q / d \delta=0)$ ). Such a "spurious dispersion" carries the sign of $h_{x}$ along with it, and so has opposite polarity for oppositely charged particles. It cannot be compensated by magnetic elements for both beams at the same time, because it is caused by electrostatic separation elements. Such a separation induced dispersion wave has been observed at CESR, where $Q_{h}=3$ with separation in one plane only, with good agreement between measurement and prediction. 15,16

The differential of the helix phase advance with respect to energy can be very large in practice, because while the intersection regions are the largest source of natural chromaticity, the bulk of the chromatic correction will come from chromatic sextupoles inside the helices. For example, assuming as elsewhere that the sextupoles are powered at three times the strength necessary 
for correction of the arc chromaticity contribution alone, the crucial variation is given by

$$
d\left(\omega_{2}-\omega_{7}\right) / d \delta=(3-1) 2 \pi 0_{h} \tan \left(\omega_{c} / 2\right) /\left(\omega_{c} / 2\right)
$$

where ${ }_{c}$ is the phase advance per cell.

Consider now two variants of the 72 degree phase advance per cell lattice discussed elsewhere, as shown in Fig. 4.21. In the "distributed" variant there are eight octants with individual tunes of $Q_{8}=12.8$, with a helix of $Q_{h}=12$ symmetrically placed in the middle of each octant. In the "clustered" variant the same "intersection regions" and helices are rearranged into two arcs, for a total tune of $Q=8 Q_{8}=102.4$, with helices of $Q_{h}=4 \times 12=$ 48 in the middle of each arc. In both variants successive helices have alternating polarities, so that the distributed and clustered lattices have superperiodicities of 4 and 1 , respectively. This symmetry allows the distributed lattice to be reduced to a more fundamental machine consisting of two octants, since only systematic effects are being considered here. The fundamental version of the distributed lattice is similar to the clustered lattice scaled down by a factor of four, with a total tune of $Q=25.6$, and with $Q_{h}=12$ for each of the two helices.

The helices and the dispersions are mirror symmetric about a line drawn through diametric points with $=0$ and $\approx 0$, symmetrically placed between the two helices. Combining equations 4 and 5 , the dispersion is given by

$$
n_{x}=h_{x}\left(2 \pi Q_{h} / \cos (\pi Q / 2)\right)\left(\tan \left(\phi_{c} / 2\right) /\left(\sigma_{c} / 2\right)\right)\left(B / B_{\max }\right)^{1 / 2} \cos \theta
$$




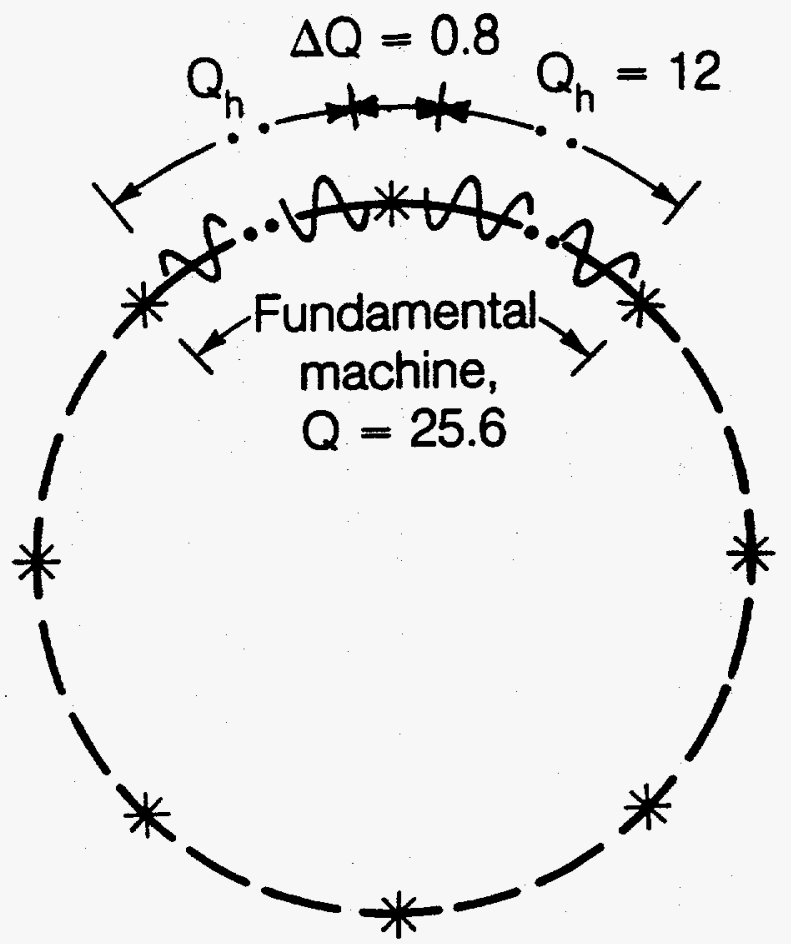

a) Distributed Lattice

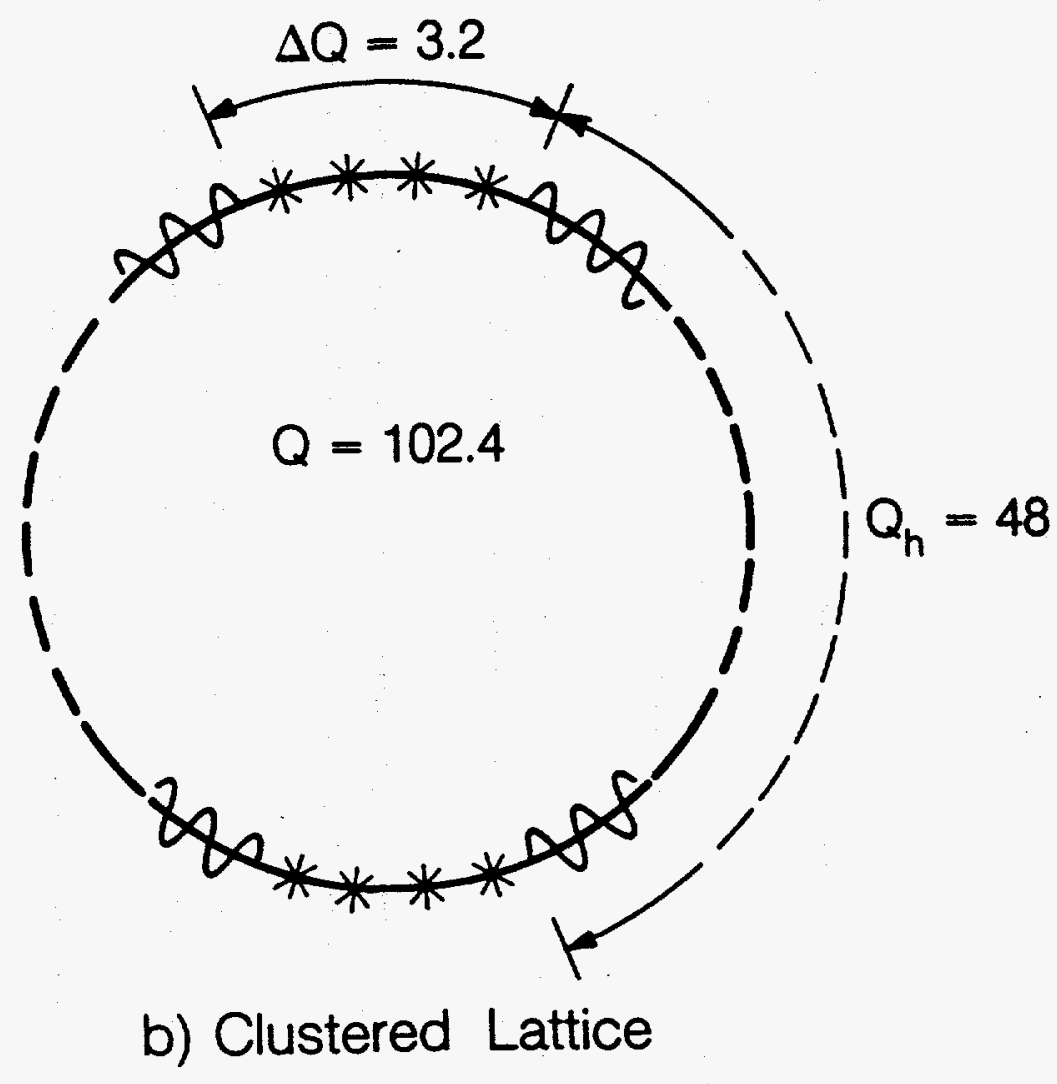

Fig. 4.21 Distributed and clustered variants examined for spurious dispersion effects. 
The amplitude of the spurious dispersion outside the helices is therefore given, for the two lattice variants, by

$$
\begin{aligned}
& n_{\max }=1.08[h / 0.01] \text { meters (distributed), } \\
& =4.31[\mathrm{~h} / 0.01] \text { meters (clustered). }
\end{aligned}
$$

where the helix radius is measured in meters. For example, a helix with a one centimeter maximum radius produces, in the clustered lattice, a spurious dispersion wave which is larger than the peak natural dispersion of 3.41 meters and has the same amplitude in both the horizontal and vertical planes. A similar analysis can be performed for the spurious dispersion inside the helices, giving

$$
n_{x}=h_{x} 2 \pi Q_{h} \tan (\pi Q / 2)\left(\tan \left(\theta_{c} / 2\right) /\left(\sigma_{c} / 2\right)\right)\left(B / B_{\max }\right)^{1 / 2} \sin \sigma_{.}
$$

where the phase origin has been moved to the center of a helix.

Inside the helices the amplitude of the spurious dispersion is then

$$
\begin{array}{rlr}
n_{\max } & =0.63[\mathrm{~h} / 0.01] \text { meters } & \text { (distributed), } \\
& =2.53[\mathrm{~h} / 0.01] \text { meters } & \text { (clustered) }
\end{array}
$$

and once more the spurious dispersion in clustered lattices is comparable with the natural dispersion.

One obvious damaging effect caused by these dispersion waves is the increase in magnet aperture which is required. Another effect is the modification of partition numbers, $J_{j}$, which describe the radiation damping times through the equations

$$
\begin{array}{ll}
\tau_{i}=(2 \mathrm{TE} / U) / J_{i} & i=h, v \text {, or } \mathrm{s}, \\
J_{h}=1-0, \quad J_{v}=1, J_{s}=2+0
\end{array}
$$


Here $T$ is the revolution period, $E$ is the design energy, $U$ is the nominal energy loss per turn, and the horizontal and synchrotron partition numbers are related through the parameter 0 , which is well approximated in the case at hand by

$$
D=2 \iint_{Q} n h k^{2} d s / \int_{D} G^{2} d s
$$

These integrals are over all quadrupoles or all dipoles, with $\mathrm{K}=2.38 \times 10^{-3} \mathrm{~m}^{-2}$ the quadrupole field gradient, and $G=8.62 \times 10^{-5} \mathrm{~m}^{-1}$ the dipole bending strength.

The numerator in equation (11) is ideally zero, because while the design dispersion does not nominally vary from one (de)focusing quadrupole to the next, the horizontal helix displacement $h$ oscillates with the betatron phase. However, the spurious component of the dispersion also oscillates with the betatron phase, with an amplitude $\eta_{\max }$, so that the numerator is non-zero, and $D$ is we 11 approximated by

$$
D=N_{C}\left(1+B_{\min } / B_{\max }\right) n_{\max } h_{x} K^{2} L_{Q} / 2 \pi G
$$

Here $N_{C}=480$ is the number of cells in the helices of the prototype lattices, and $\mathrm{L}_{Q}=5.0$ meters is the length of a quadrupole. Substituting equation (9) into equation (12), the variation of 0 with helix amplitude is

$$
\begin{aligned}
D & =0.20[\mathrm{~h} / 0.01]^{2} & & \text { (distributed), } \\
& =0.80[\mathrm{~h} / 0.01]^{2} & & \text { (clustered), }
\end{aligned}
$$

up to a sign. A separation helix in a clustered lattice with a radius near one centimeter can therefore drastically modify the partition numbers, and the synchrotron radiation damping times. 


\subsubsection{Magnet Bore Requirements}

Tracking results are reported above for a lattice with a peak separation helix radius of 7.5 millimeters in the presence of random multipoles, with strengths which were scaled down from a dipole with a 2.0 centimeter radius to those of a dipole with a 2.75 centimeter radius. Two criteria are used here to make a more realistic estimate of the increased bore size necessary for proton-antiproton operation of the SSC. First, the phase space dilution of the beam due to injection off the central axis should not be significant, using the same horizontal and vertical damping systems that are assumed for protonproton loading. Second, all persistent current systematic multipoles above decapole must be small enough, at injection, that each induces a horizontal tune shift of less than 0.005 to a particle with a betatron amplitude of 0.5 centimeters horizontally, and a relative energy error of 0.001 . The latter requirement is the more stringent, producing a bore radius which is consistent with the value used for tracking purposes.

\subsubsection{Injection Tracking Studies}

One requirement on the needed aperture for a proton-antiproton collider is that the expected injection errors at $1 \mathrm{TeV}$ do not cause an unacceptable amount of beam dilution by coherent oscillations and filamentation. A computer simulation program has been written which sets up a test SSC lattice composed of F000 cells, dispersion supressors, and two clustered straight sections. This program has been modified to allow for a closed orbit deformation as required for proton-antiproton operation, with the original damping system now operating about the off-axis closed orbit. The dipoles are given randomly generated 
errors $a_{1}$ through $a_{10}$, and $b_{1}$ through $b_{10}$, as defined by the MagneticErrors Working Group ${ }^{17}$, and are then sorted in lots of 60 on $b_{2}$. Ensembles of 500 particles are then randomly generated and injected into the lattice with typical injection errors, which are assumed to be

$$
\begin{aligned}
& \text { Horizontal error }=1.5 \text { millimeters, } \\
& \text { Vertical error }=1.5 \text { millimeters, } \\
& \text { Momentum error }=1.5 \times 10^{-4} .
\end{aligned}
$$

The particles are tracked for 100 turns while being subjected to slow horizontal and vertical damping systems with 50 turn characteristic times. Proton-proton SSC lattice results have already been discussed ${ }^{18}$. The increases in horizontal and vertical beam sizes for the proton-antiproton case are shown in Table 4.5, where they are compared with those of the proton-proton case. The dipole errors come from dipoles with a bore radius of 2.0 centimeters, for two different random number seeds.

Table 4.5

RMS beam size increase due to injection errors
$\Delta \sigma_{X}$
$\Delta \sigma_{y}$

Case

pp

$\bar{p} p$

pp

$\overline{p p}$

1

$1.7 \%$

$6.4 \%$

$14.3 \%$

$26.3 \%$

2

$5.6 \%$

$36.6 \%$

$2.8 \%$

$54.0 \%$ 
The proton-proton machine has an on-axis closed orbit while the protonantiproton machine has a closed orbit separation of $\pm 7.5 \mathrm{millimeters.} \mathrm{In} \mathrm{the}$ proton-antiproton case it has been necessary to determine the actual closed orbit to within a few microns in order to have the damping system work as well as it does. A closed orbit error of \pm 0.2 millimeters is enough to double the beam size increases from those shown.

The rms beam size increases are consistently larger for the protonantiproton case than for the proton-proton case, for both random number seeds. The largest proton-proton increase is $14.3 \%$ vertically, for the first seed, while the largest proton-antiproton increase is $54.0 \%$ vertically, for the second seed. These numbers show that beam dilution at injection may not be ignored in the proton-antiproton case, although it is not unacceptable. The dilution will become less important as the dipole bore is increased for other reasons, and in the last resort could be better controlled by strengthening the injection damping system. Beam dilution at injection is not a critical effect in determining the required magnet bore size.

\subsubsection{Systematic Tune Shifts}

There must be a sufficiently large linear aperture for successful operation of the $\mathrm{SSC}^{19}$. This condition places constraints on acceptable field errors in the dipoles, in particular on the $b_{2 m}$ parameters which describe the strength of systematic multipoles with the 'allowed' mid-plane symmetry. The linear aperture necessary for satisfactory operation was defined in the conceptual Design Report by demanding that a trajectory with a peak horizontal amplitude of $A_{B}=0.5$ centimeters, and an relative energy offset of $\delta=$ 0.001 , should not have a tune shift of greater than $\Delta v_{\max }=0.005$, from any 
individual systematic multipole. If one particular value of $b_{n}$ is too high, according to this criterion, one way to compensate it is by adding distributed multipole windings to the dipoles. This is impractical for more than a small number of multipole components, because it complicates both the construction of the magnets and the operation of the storage ring. Another way to decrease the tune shift is to increase the bore radius, since the multipole strengths are expected to scale according to $b_{n} \sim r_{\text {bore }}^{-n-1 / 2}$.

In the case of a proton-proton SSC, without orbit separation, the horizontal tune shift is given by ${ }^{20}$

$$
\Delta v=b_{n}<\beta \cos \emptyset\left(n \delta+A_{\beta}\left(B / B_{\max }\right)^{1 / 2} \cos \theta\right)^{n}>/ A_{\beta}
$$

where the angle brackets $\ll$ imply averaging over both the betatron oscillation phase, 6 , and the variation of beta and dispersion functions inside a FODO ce11. One of these averages may be removed for the sake of simplicity by using the 'smooth' approximation, in which $B$ and $n$ are constants and the phase advances at a constant rate. The tune shift then becomes

$$
\Delta v=b_{n} B \sum_{i}\{n, i\}\{i+1,(i+1) / 2\} 2^{-(i+1)} A_{B}^{i-1}(n \delta)^{n-i}
$$

where $\{j, k\} \equiv j ! /[k !(j-k) !]$ is a combinatorial factor, with on ly integral values of $j$ and $k$ allowed. The values of $b_{n}$ which correspond to a tune shift of 0.005 , according to this equation, are recorded in COR Table 4.4-3. Comparison of these numbers with the calculated and measured systematic multipole moments for the nominal SSC magnet design ${ }^{20}$, with a 2.0 centimeter bore, shows that the uncorrected sextupole and decapole moments, $b_{2}$ and $b_{4}$, are too large. The CDR proposal is to correct them with distributed windings. The $b_{6}$ component is marginal, while higher order multipoles are significantly less critical. 
When a one dimensional separation of amplitude $h_{x}$ in the horizontal plane is added, for a peak total separation of $2 h_{x}$, the horizontal tune shift in the smooth approximation becomes

$$
\begin{gathered}
\Delta v=b_{n} B<\cos \theta\left(h_{x} \cos \phi_{x}+n \delta+A_{B} \cos \theta\right)^{n}>/ A_{B} \\
=b_{n} B \sum_{i, j}\{n, i\}\{i+1,(i+1) / 2\}\{n-i, j\}\{j, j / 2\} 2^{-(i+j+1)} A_{B}^{i-1} h_{x}^{j}(n \delta)^{n-i-j}
\end{gathered}
$$

where the average is taken independently over the oscillation phase, and $6_{x}$, the encounter region phase. The tune shift with full helical separation, before making the smooth approximation, is

$$
\Delta v=b_{n}<B \cos \theta \operatorname{Re}\left(z^{n}\right)>/ A_{B}
$$

with

$$
\begin{aligned}
z=A_{B}\left(\beta_{x} / B_{\max }\right)^{1 / 2} \cos \phi_{x}\left(\beta_{x} / B_{\max }\right)^{1 / 2} \cos \sigma_{x}+\eta \delta \\
+i h_{y}\left(B_{y} / B_{\max }\right)^{1 / 2} \sin \phi_{y}
\end{aligned}
$$

where the average is again taken over the encounter region phases, and $\sigma_{y}$, independently of the oscillation phase. It is readily shown that the tune shift is zero if the smooth approximation is applied to equation 4 , when the horizontal and vertical amplitudes, $h_{x}$ and $h_{y}$, are equal. It is therefore necessary to evaluate the systematic tune shift in the presence of helical separation without resorting to the smooth approximation, a calculation which is only practical by numerical integration.

Figure 4.22 shows the results of using such a calculation, in conjunction with the expected scaling of the multipole strengths, to infer the minimum bore radius for a proton-antiproton SSC, with the nominal multipole strengths listed in the COR as a reference standard. The solid curves show the radius necessary to reduce the tune shift due to $b_{6}, b_{8}$ and $b_{10}$ down to the 


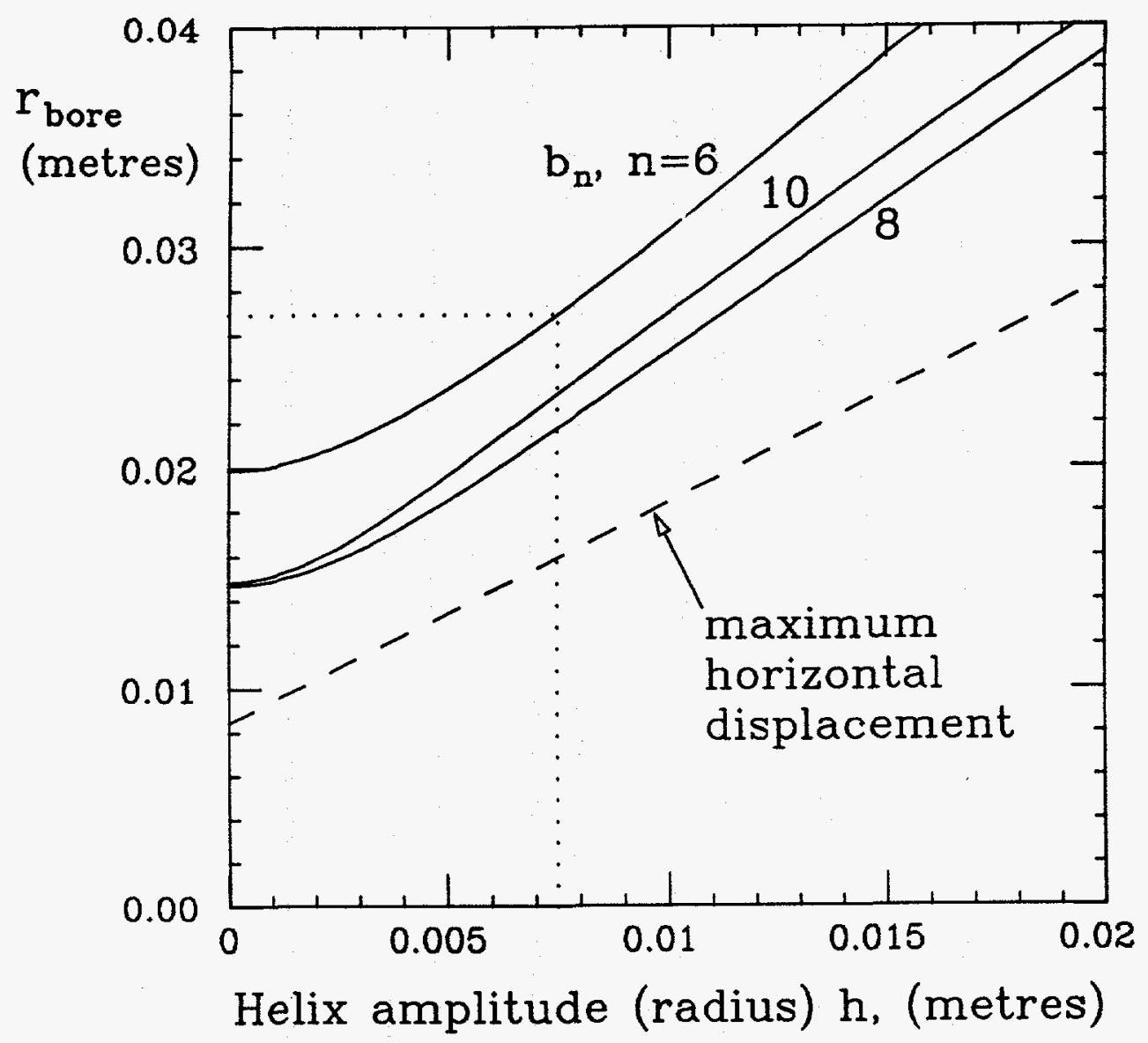

Fig. 4.22 The minimum bore radius necessary to decrease the systematic multipole, $b_{n}$, to acceptable levels, as a function of helix radius.

maximum acceptable level of 0.005 , as a function of the helix amplitude, for a nominal machine made of 96 meter cells with a phase advance of 72 degrees per cell. The dashed straight line is a line of maximum horizontal displacement,

$$
x_{\max }=A_{B}+n_{\max } \delta+h_{x}=0.005+0.0034+h_{x} \text { meters }
$$

representing the required linear aperture. Each of the solid curves is roughly in a constant ratio with the dashed line. Thus, as a rule of thumb, the situation may be casually summarized by saying that the maximum excursion of a particle must always remain inside a good field region, which is empirically found to extend out to about $60 \%$ of the inner coil radius. This makes it possible to estimate the extra bore size required to allow for further realistic effects, or for errors which proton-antiproton operation will incur. 


\subsubsection{Beam and current limitations}

The total beam current is decreased in a proton-antiproton SSC, compared with the proton collider described in the Conceptual Design Report, and all effects depending on the total current are much weaker. On the other hand the single bunch current may be larger, as in the extreme case $B$, since the bunch number of bunches is considerably reduced. In the nominal case A, however, even the single bunch population is slightly reduced, as shown in Table 4.6.

Single bunch or single beam current effects therefore do not have a major impact on the nominal proton-antiproton design. For example, the total synchrotron power radiation decreases from the proton-proton value of $16.8 \mathrm{~kW}$ to $2.2 \mathrm{~kW}$. The power loss per beam due to the parasitic heating of impedance sources also decreases in all cases, since not only are the currents smaller, but also the resistive wall impedance and the bellows impedance decrease in

Table 4.6

\begin{tabular}{lccc} 
& $\begin{array}{c}\text { Number of } \\
\text { bunches } \\
\text { B }\end{array}$ & $\begin{array}{c}\text { Bunch } \\
\text { Population } \\
N_{b}\left[10^{10}\right]\end{array}$ & $\begin{array}{c}\text { Total } \\
\text { Population } \\
\text { NT[1013] }\end{array}$ \\
\cline { 2 - 4 } proton-proton(CDR) & 17280 & 0.73 & 12.0 \\
Case A & 2400 & 0.64 & 3.1 \\
Case B & 1200 & 1.28 & 3.1
\end{tabular}

inverse proportion to the increased chamber diameter. Intrabeam scattering lifetimes increase slightiy, because the phase space density decreases. Impedance thresholds for single and multi-bunch effects increase with the inverse of the nominal bunch population. 


\subsubsection{Effect of Ions}

The proton and antiproton beams ionize the molecules of the residual gas in the vacuum system. Under certain conditions an accumulation of ions in the antiproton beam may occur, which in turn leads to an increase of the local gas pressure within the beam, and to space charge effects. It should be mentioned that since the two beams are separated they can be treated independently, and no ions will be trapped by the proton beam.

The conditions for ion accumulation to start are investigated here. A linear approximation for the interaction of the ions with the beam is used. The linear theory leads in general to pessimistic results, with critical masses below the true values. 21

The conditions to trap ions were investigated for cases A, B, and B-1, which are defined by the parameters given in Table 4.4 above.

The change in transverse velocity for an ion which is created within a beam of elliptical cross section and uniform particle distribution is given, for small displacements in one plane, by

$$
d y / d t=-a_{y} y \quad(y=z \text { or } x)
$$

with

$$
\begin{aligned}
& a_{z, x}=2 r_{p} C N_{B} / A \sigma_{z, x}\left(\sigma_{z}+\sigma_{X}\right) \\
& c=\text { velocity of light } \\
& r_{p}=\text { classical proton radius } \\
& N_{B}=\text { particles per bunch } \\
& A=\text { ion mass/proton mass } \\
& \sigma_{X}=\text { horizontal beam half size } \\
& \sigma_{Z}=\text { vertical beam half size }
\end{aligned}
$$


For a beam with equally spaced bunches and identical particle populations one period of forces on the ion is given by the kick described above, followed by a drift according to the bunch distance i.e.

$$
M=\left(\begin{array}{cc}
1 & t_{B} \\
0 & 1
\end{array}\right)\left(\begin{array}{cc}
1 & 0 \\
-a_{y} & 1
\end{array}\right)
$$

with $t_{B}$ the time separation between the bunches.

The motion is stable and ions are trapped if

$$
-2<\operatorname{Tr}(M)=2-a_{y} t_{B}<2
$$

Using relation (2) for the kick parameter, the limit expressed by (4) is reached for the critical ion mass

$$
A_{C}=r_{p} C_{0} N_{B} / 2 B \sigma_{y}\left(\sigma_{x}+\sigma_{z}\right)
$$

where $C_{0}$ is the circumference of the ring and $B$ is the number of bunches.

To calculate the critical masses for the pp SSC, the geometry factor

$$
g(x, z)=\max \left\{1 / \sigma_{x}\left(\sigma_{x}+\sigma_{z}\right), 1 / \sigma_{z}\left(\sigma_{x}+\sigma_{z}\right)\right\}
$$

was evaluated around the ring for equal emittances in both planes at an energy of $1 \mathrm{TeV}$, i.e. $\varepsilon_{x}=\varepsilon_{z}=10^{-9} \mathrm{~m}$. We obtain the following minimum value:

$$
g(x, z)=0.389 \times 10^{7}\left[\mathrm{~m}^{-2}\right]
$$

(a) Multi-bunch mode with equidistant bunches.

For complete filling with equidistant bunches the critical masses are below the atomic mass of carbon dioxide. As we see from Table 4.7 the ions will always be trapped.

Table 4.7

$\begin{array}{llll}\text { Case } & \text { A } & \text { B } & \text { B-1 } \\ \text { Critical mass } & 0.8 & 1.6 & 3.1\end{array}$


(b) Multi-bunch filling with a gap.

If a gap is introduced in the beam, the periodicity of the forces is reduced. Resonances are excited which generate unstable bands within the ion mass spectra similar to the half integer resonance in an accelerator when quadrupole distortions are introduced.

The stability of the ions has been analyzed by calculating the trace of the transformation for one period of force, which is now

$$
M=\left(\begin{array}{ll}
1 & t_{G} \\
0 & 1
\end{array}\right)\left[\left(\begin{array}{cc}
1 & t_{B} \\
0 & 1
\end{array}\right)\left(\begin{array}{cc}
1 & 0 \\
-a_{y} & 1
\end{array}\right)\right]^{B}
$$

with $t_{G}$ the gap length.

The gap length in the beam required for beam abortion is roughly $3 \mu \mathrm{s}$. It is assumed to be larger for the $\overline{p p}$ option to provide injection of the beam batches without affecting the counter-rotating beam.

The influence of a gap on ion stability has been calculated here for $5 \mu \mathrm{s}$ and 10us gap size; these gaps give 18 and 37 missing bunches out of 1200 for both cases $B$ and $B-7$, while for case $A$ these numbers are doubled.

Figure 4.23 shows the stability of ion masses for case $B$. For a $5 \mu \mathrm{s}$ gap there are (theoretically) five discrete trapped ion masses below 50, falling to two if the gap is increased to $10 \mu \mathrm{s}$.

The same graph is shown in Fig. 4.24 for case B-1. The increased bunch currently slightly reduces the ion stability.

Figure 4.25 shows the results for case $A$. Even though the number of bunches is increased (and compared to case $B$ the bunch current is reduced) the stability behavior hardly differs from the two previous cases.

The results indicate that even in linear theory most of the ion masses will not be trapped if a gap is introduced. For all three investigated cases a gap of $5 \mu$ s seems to be sufficient to get rid of the ions. If an ion mass 
Case B

$U p=$ Unstable ion mass

Down $=$ Stable ion mass

$\mathrm{Gap}=5 \mu \mathrm{S}$

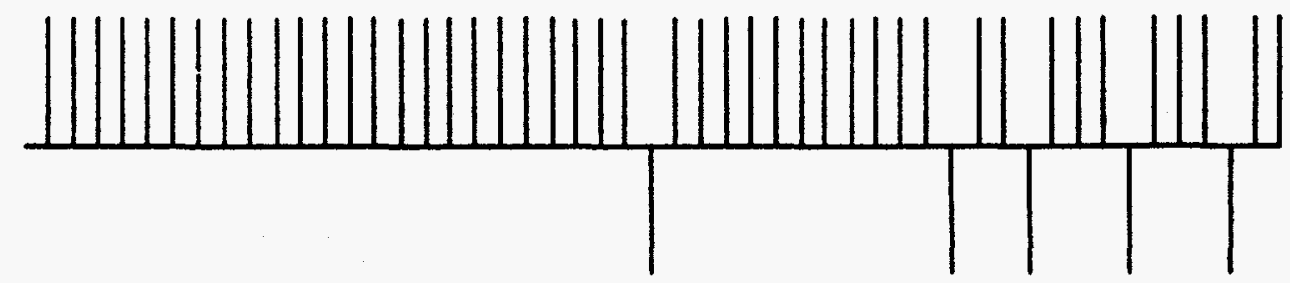

Gap $=10 \mu \mathrm{s}$
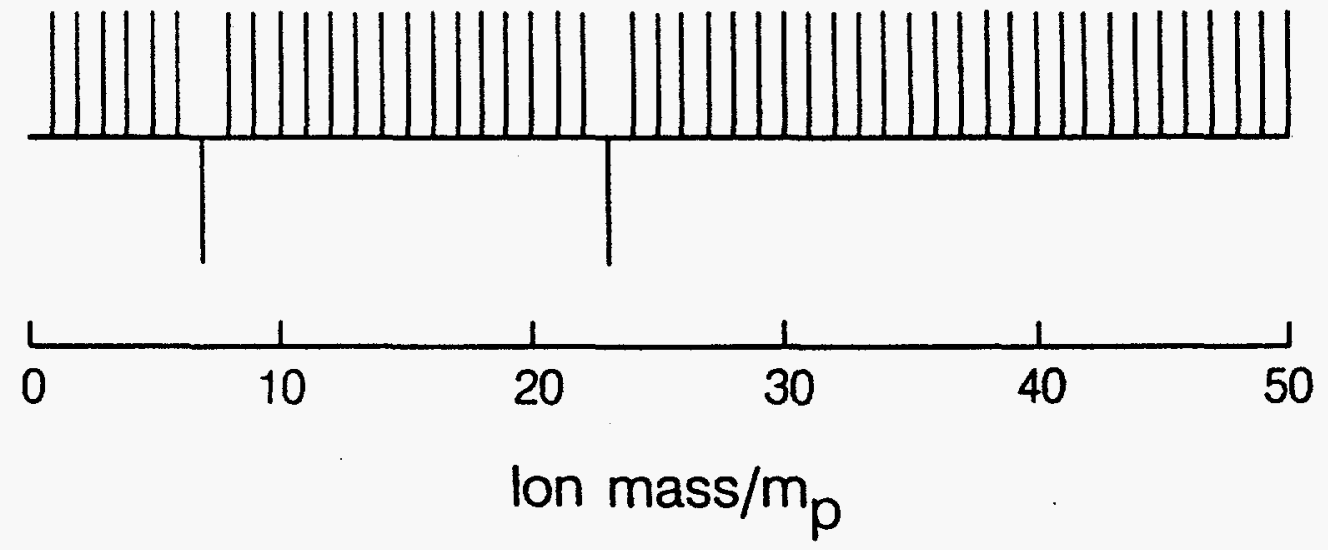

XBL 865-10781

Fig. 4.23 Ion stability vs. ion mass (case B). 
Case B-1

$U p=$ Unstable ion mass

Down $=$ Stable ion mass
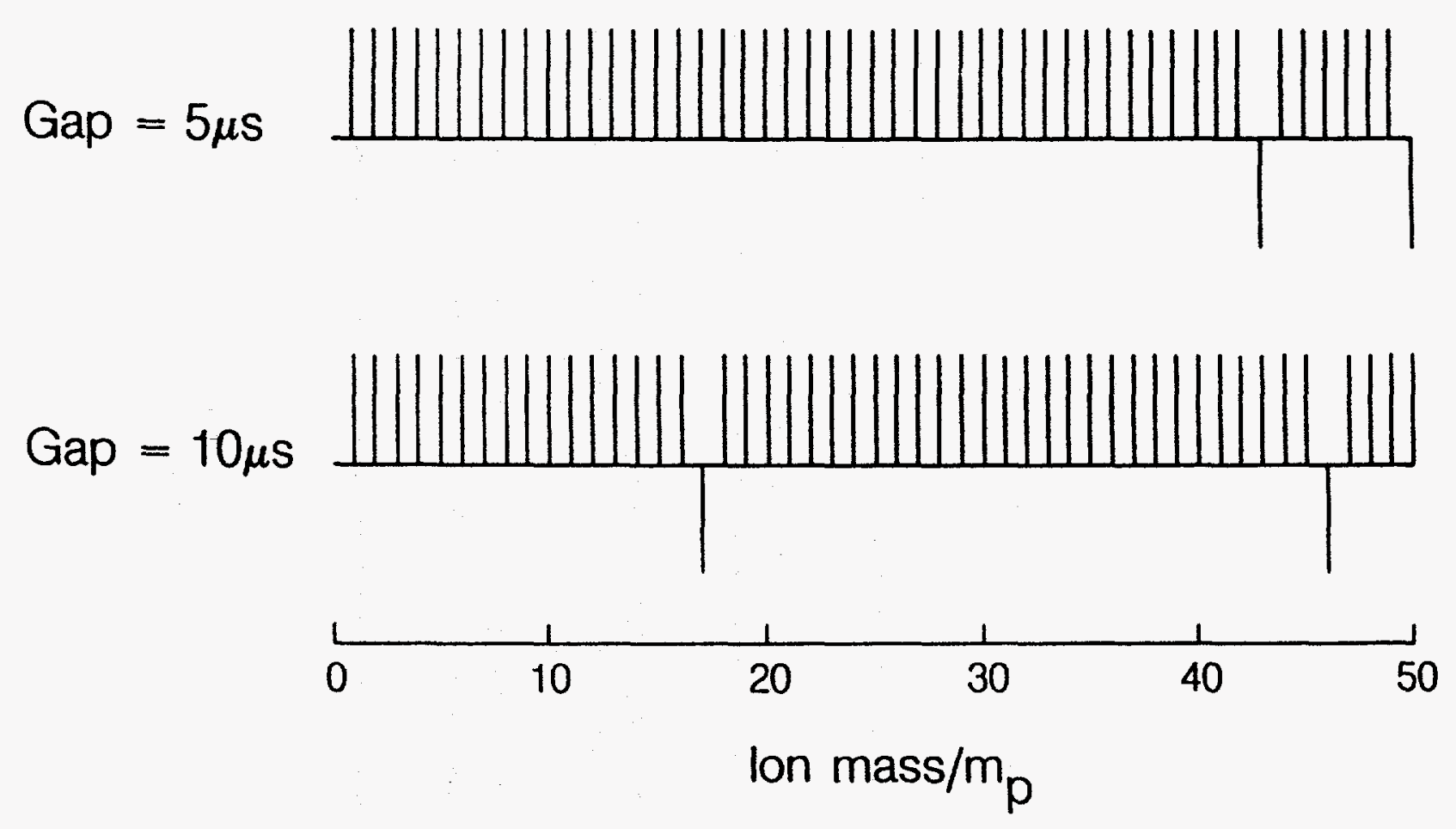

Fig. 4.24 Ion stability vs. ion mass (case B-1) 
Case A

Up = Unstable ion mass

Down $=$ Stable ion mass

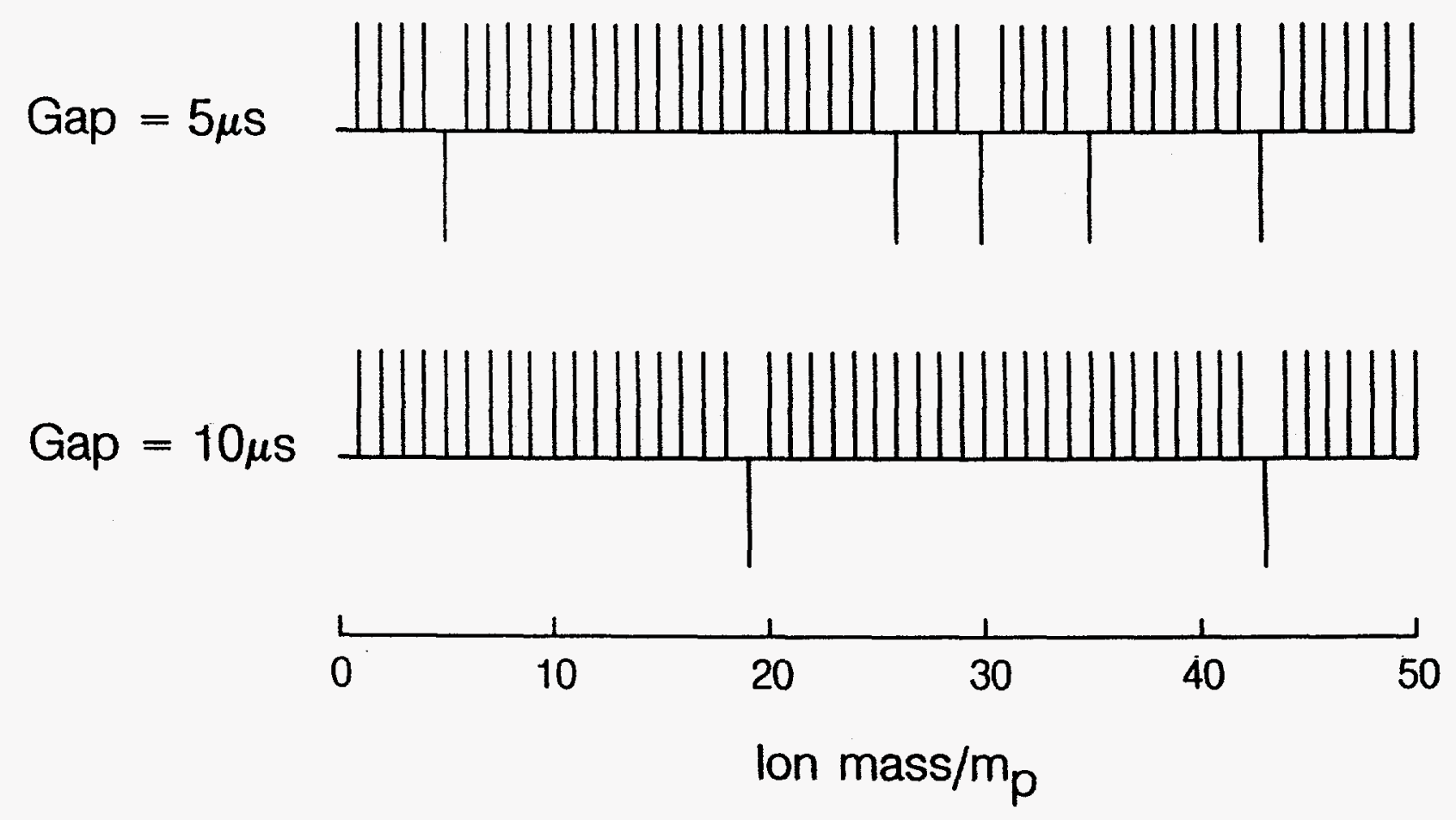

Fig. 4.25 Ion stability vs, ion mass (case A). 
falls within a stable band, ions will only be accumulated until the neutralization reduces the forces to a limit where the ion is shifted into the unstable region. No ion ladder can be built up.

\subsubsection{Conclusions}

The feasibility of proton-antiproton operation for luminosities of $0.5 \times 10^{32}, 10^{32}$ and $10^{33} \mathrm{~cm}^{-2} \mathrm{~s}^{-1}$ has been investigated.

The minimum allowable separation of the two beams circulating in the same vacuum chamber is essentially defined by dynamic aperture requirements in the presence of long range beam-beam interactions. The mutual beam-beam tune shift, the tune spread with amplitude, and the chromaticity distortions are important effects, but are not critical.

The tune shifts generated around the separated closed orbits by feed-down effects of random multipoles and chromaticity correcting sextupoles are large, and must be compensated. A compensation scheme which uses two sextupoles per encounter period has been tested, and appears to be adequate.

An antiproton production rate of $2 \times 10^{8} \mathrm{~s}^{-1}$ is necessary to provide the nominal luminosity of $10^{32} \mathrm{~cm}^{-2} \mathrm{~s}_{1}^{-1}$ and to reduce the average number of events per crossing to 1.5. The lower limit to the separation recommended for the two beams is 15.0 millimeters, in a helical separation scheme. If an antiproton production rate of only $10^{8} \mathrm{~s}^{-1}$ can be achieved, the luminosity is correspondingly reduced to $0.5 \times 10^{32} \mathrm{~cm}^{-2} \mathrm{~s}^{-1}$ for an increased tune shift parameter of 0.0016 . The required beam separation remains the same.

The separation helices in a proton-antiproton SSC cause spurious dispersions of similar amplitudes in both vertical and horizontal planes, with a different polarities for each beam; these cannot be corrected magnetically. 
The amplitude of the dispersion wave so caused is proportional to the rate of change of phase advance across the helix with respect to energy, and so is proportional to the length of the helix. A clustered lattice is therefore significantly more vulnerable than a distributed lattice to the dynamical ill effects caused by this dispersion. An analysis of a representative clustered lattice shows that the spurious dispersion amplitude is typically about the same size as the design dispersion. These facts point very strongly to the use of a distributed lattice, rather than a clustered lattice, in a protonantiproton SSC. The spurious dispersion is still significant in a distributed lattice.

The dipole bore must be increased in order for a proton-antiproton SSC to perform satisfactorily. Most critical is the need to maintain the linear aperture during injection at the value specified in the CDR, in spite of persistent current-induced systematic multipoles. This leads to the requirement that the bore radius must be 2.69 centimeters if the dipoles are to accommodate a 7.5 millimeter radius helix. This estimate does not include any allowance for the helix to be slightly larger than the recommended minimum radius, and does not include any margin to absorb the secondary dynamic effects which separated orbit operation will induce. The required bore radius is increased by 0.13 centimeters, to 2.82 centimeters, when a 1.08 meter amplitude spurious dispersion wave is included in a distributed lattice. These estimates are in reasonable agreement with the scaling of linear aperture with bore radius which has been observed in tracking programs. This scaling predicts that a radius of $3.05 \pm 0.38$ centimeters is required, when extrapolated slightly beyond its range of applicability 22 .

To achieve a luminosity of $10^{33} \mathrm{~cm}^{-2} \mathrm{~s}^{-1}$, the antiproton production rate must be increased to $10^{9} \mathrm{~s}^{-1}$, and a tune shift parameter of $\xi=0.003$ must be anticipated, close to the empirical limit. The influence of the long range 
beam-beam effect on beam dynamics is tremendous and requires a total beam separation of 50 millimeters for an adequate dynamic aperture. Since the magnet aperture must be increased with the separation amplitude, this example is outside the parameter range where a cost benefit can be expected by using only one ring.

Parameters for the four different cases which have been studied, including the nominal case $A$, are collected in Table 4.8.

Table 4.8

The main parameters for four representative proton-antiproton storage rings.

\begin{tabular}{|c|c|c|c|c|}
\hline & A & B & $c$ & D \\
\hline Luminosity $/ 70^{32} \mathrm{~cm}^{-2} \mathrm{~s}^{-1}$ & 1.0 & 1.0 & 0.5 & 10.0 \\
\hline max. beam separation/mm & 15 & 15 & 15 & 50 \\
\hline$\overline{\mathrm{p}}$ production rate $/ 10^{8} \mathrm{~s}^{-1}$ & 2.0 & 2.0 & 1.0 & 10.0 \\
\hline head on bb tune shift $/ 10^{-3}$ & 0.8 & 1.6 & 1.6 & 3.0 \\
\hline antiprotons per bunch $/ 10^{10}$ & 0.64 & 1.28 & 1.28 & 0.85 \\
\hline protons per bunch/10 10 & 0.64 & 1.28 & 1.28 & 2.56 \\
\hline number of bunches & 1200 & 2400 & 600 & 9600 \\
\hline events per crossing & 1.6 & 3.2 & 3.2 & 4.3 \\
\hline bunch spacing/m & 40 & 80 & 160 & 10 \\
\hline collision betaim & 0.5 & 1.0 & 1.0 & 1.0 \\
\hline
\end{tabular}


References

1. $\overline{p p}$ Options for the Supercollider, Proceedings of a workshop organized by Argonne National Laboratory and the University of Chicago, Feb. 1984, Chicago, Illinois

2. Design and Utilization of the SSC, Proceedings of the 1984 Snownass Summer Study, June/July 1984, Snowmass, Colorado

3. C. Johnson and C. Hojvat, in ref. 7, p.326

4. Conceptual Design of the Superconducting Super Collider, SSC-SR-2020, SSC Central Design Group, March 1986.

5. C. Ankenbrandt, The Evolution of Luminosity and the $\overline{p p}$ Option, ref. 2

6. J. Gareyte, Performance of the CERN SPS as a Proton-Antiproton Collider, CERN SPS/83-28 (1983).

7. S. Peggs, The Proton-Antiproton Option, ref. 2

8. S. Peggs, Double Helix Separation in the SSC, ref. 2

9. A. Ruggiero, Beam-Beam Tune Shift and Tune Spread for a pp Collider with Separation in one Plane, ref. 2

10. R.R. Wilson, Momentum Separation of Proton and Antiproton Beams in a Collider, ref. 2

11. T.L. Collins, A High Luminosity pp-Ring, ref. 1

12. A.G. Ruggiero, A Scenario for a Proton-Antiproton Collider at $20+20 \mathrm{TeV}$ and $10^{32} \mathrm{~cm}^{-2} \mathrm{~s}^{-1}$ luminosity, ref. 1

13. A. Wrutich, RACETRACK, A Computer Code for the Simulation of Nonlinear Particle Motion in Accelerators, DESY.

14. SSC Aperture Estimate for Cost Comparisons, SSC-SR-1013

15. CESR Log 58, p. 28, CESR, Cornell University (1984).

16. S. Peggs, Colliding Beam Note 84-14, CESR, Cornell University (1984).

17. E. Fisk, et al., Preliminary Report of the Magnetic-Errors Working Group of the SSC Aperture Workshop, SSC-7

18. D. E. Johnson, SSC Injection Simulation Results, SSC-60

19. COR, ref. 4 , section 4.3 
20. D. Neuffer and J. Peterson, SSC Central Design Group Internal Report No. SSC-N-135 (1986)

21. Y. Baconnier and G. Brianti. The Stability of Ions in Bunched Beam Machines, CERN/SPS/80-2(DI), March 1980.

22. CDR, ref. 4 , section 4.3 .3 
Chapter 5

\section{COST IMPLICATIONS}

\subsection{Introduction}

We have estimated the cost implications of the $\overline{p p}$ collider of $10^{32} \mathrm{~cm}^{-2} \mathrm{~s}^{-1}$ considered in this report. This has been done by comparing the $\overline{p p}$ option with the $\mathrm{PP}$ collider of $10^{33} \mathrm{~cm}^{-2} \mathrm{~s}^{-1}$ presented in the Conceptual Design Report. System by system, we have estimated the differences between the two cases for the component costs only. 


\subsection{Injector Systems}

The injector system for the pp collider consists of a source and a linear accelerator of $600 \mathrm{MeV}$, followed by three booster synchrotrons with kinetic energies of $8 \mathrm{GeV}$ (LEB), $100 \mathrm{GeV}$ (MEB), and $1 \mathrm{TeV}$ (HEB).

The scheme considered here for the $\overline{p p}$ collider makes several modifications and extensions to the above system: the MEB is upgraded from $100 \mathrm{GeV}$ to 150 GeV to increase the yield of antiprotons; the debunching ring and cooling ring use the Fermilab $8.89 \mathrm{GeV} / \mathrm{c}$ design, extrapolated to $10 \mathrm{GeV} / \mathrm{c}$; and finally, a $150 \mathrm{GeV}$ holding ring for the accumulated antiprotons is placed in the same tunnel as the MEB. The Linac and LEB are assumed to be unchanged by the small increase in bunch intensity necessary for $\bar{p}$ targeting. Below we estimate the cost of modifying the injector systems to provide both protons and antiprotons.

\subsubsection{Modifications to the MEB.}

For the $\overline{p p}$ collider considered here, we have increased the energy of the MEB to $150 \mathrm{GeV}$ and the repetition rate from 0.25 to $1 \mathrm{~Hz}$. We assume that in thus increasing the energy, the magnet aperture remains the same while the circumference of the machine is increased by $50 \%$. To accommodate the increase in energy and repetition rate, the RF system is upgraded by a factor of six. The higher repetition rate also increases the power supply demand by a factor of two. Finally, extra abort and extraction systems are both needed for the MEB for transporting the antiprotons to the holding ring.

The estimated increases in costs for the various subsystems are given below in Table 5.1. For comparison we list the CDR costs for same subsystems. 
Table 5.1

MEB Component Costs (FY86 k\$)

\begin{tabular}{|c|c|c|c|}
\hline Item & COR & $\overline{\mathbf{p} p}$ & Ratio \\
\hline Magnets & 13,492 & 19,748 & 1.46 \\
\hline Vacuum & 979 & 1,379 & 1.5 \\
\hline Instrumentation & 550 & 825 & 1.5 \\
\hline Controls & 1,027 & 1,541 & 1.5 \\
\hline Safety & 276 & 414 & 1.5 \\
\hline Installation & 2,968 & 4,452 & 1.5 \\
\hline Extraction & 829 & 1,244 & 1.5 \\
\hline Abort & 583 & 1,166 & 2.0 \\
\hline Injection & 217 & 434 & 2.0 \\
\hline Power Supplies & 7,101 & 14,202 & 2.0 \\
\hline R.F. & 3,750 & 22,548 & 6.0 \\
\hline TOTAL & 37,720 & 67,953 & \\
\hline
\end{tabular}

\subsubsection{Debuncher, Accumulator, and Beam Transport}

To estimate the cost of these systems, we have compared them with similar existing systems at Fermilab. We have scaled costs according to the ratio of operating energies (1.12) for the debuncher, accumulator and beam transport system.

The average cost per watt for the TeV I debuncher and accumulator stocastic cooling systems is $\$ 0.91 \mathrm{k}$. For the system under consideration here, we assume that the stocastic cooling system costs scale with the power requirements. Since the debuncher requires $10 \mathrm{~kW}$ and the accumulator $25 \mathrm{~kW}$, the incremental costs of this extra power, over the TeV I figures, are $\$ 5.6 \mathrm{M}$ for the debuncher and $\$ 16.03 M$ for the accumulator. 
In addition, there is a $15 \%$ increase in debuncher magnet cost. This is due to the greater transverse and longitudinal acceptances, which result in an aperture enlargement of $15 \%$ in the vertical plane and $20 \%$ in the horizontal plane. This modification implies an incremental price increase of $\$ 1.537 \mathrm{M}$ for the debuncher over the TeV I cost.

In Table 5.2 we summarize the costs associated with the $\bar{p}$ source assuming that the target will be a copy of the present TeV I system and that there is no additional expense associated with transporting a $150 \mathrm{GeV}$ beam to the target, instead of the $120 \mathrm{GeV}$ beam used by TeV I.

\subsubsection{Holding Ring}

Finally, the $\overline{\mathrm{pp}}$ collider requires a holding ring for the antiprotons. Although use of the HEB for storing the antiprotons was suggested in the Chicago pp workshop, we believe that for reliable operation a separate storage ring will be necessary. In any case, other uses of the HEB, such as production of beams, are anticipated. For the purpose of this study we assume an additional ring operating in the same tunnel as the MEB. In Table 5.3 we summarize the costs associated with the $150 \mathrm{GeV}$ holding ring, taking it to be a DC version of the $150 \mathrm{GeV}$ MEB. 
Table 5.2

$\bar{p}$ Source Estimate $(F Y 83-84 \mathrm{k} \$)^{*}$

\begin{tabular}{|c|c|c|c|c|}
\hline Component & TeV I & SSC & Cost Estimate & Cost Scaling \\
\hline Target System & 3,898 & 3,898 & Identical Systems & 1.0 \\
\hline Beam Transport & 11,080 & 12,401 & $\begin{array}{l}\text { Energy increased from } \\
8.89 \text { to } 10\end{array}$ & 1.12 \\
\hline Debuncher & 20,158 & 29,744 & $\begin{array}{l}\text { Energy increased from } \\
8.89 \text { to } 10 \mathrm{GeV} \\
\text { Larger Magnets } \\
\text { Larger Cooling Power } \\
\text { Requirements }\end{array}$ & 1.47 \\
\hline Accumulator & 28,649 & 48,117 & $\begin{array}{l}\text { Energy increased from } \\
8.89 \mathrm{GeV} \text { to } 10 \mathrm{GeV} \\
\text { Larger Cooling Power } \\
\text { Requirements }\end{array}$ & 1.68 \\
\hline $\begin{array}{l}\text { Conventional } \\
\text { Construction }\end{array}$ & 12,604 & 14,116 & $\begin{array}{l}\text { Energy increased from } \\
8.89 \text { to } 10 \mathrm{GeV}\end{array}$ & 1.12 \\
\hline TOTAL & 76,389 & $\begin{array}{l}108,276 \\
121,918\end{array}$ & $\begin{array}{l}83-84) \\
86)^{\star}\end{array}$ & 1.42 \\
\hline
\end{tabular}

Table 5.3

Holding Ring Component Costs (FY86 K\$) Based on the $150 \mathrm{GeV}$ MEB Estimate

Magnets
Vacuum
Instrumentation
Controls
Safety
Installation
Extraction
Abort
Injection
Power Supplies
R.F. TOTAL

TOTAL

$$
\begin{array}{r}
19,748 \\
1,379 \\
825 \\
1,541 \\
414 \\
4,452 \\
415 \\
583 \\
217 \\
14,202 \\
1,000 \\
44,776
\end{array}
$$




\subsection{Main Ring}

The major cost implication of the $\overline{p p}$ collider for the main ring is the saving which comes from the use of a single ring only. Below we detail the cost estimates associated with the main ring.

\subsubsection{Magnet System}

The cost of the magnet system is affected by several factors: a) magnet bore; b) tooling costs; c) dipole cost and quantity; d) quadrupole cost and quantity; e) spool cost; f) special IR magnets; and g) installation, survey and alignment.

Since there is only one ring of magnets in this design, we only need half the number of dipoles and quadrupoles of the CDR. However, the magnet bore must be increased to allow for the separation of the proton and antiproton beams. From ref. 1 we find that increasing the bore from $40 \mathrm{~mm}$ to $56.2 \mathrm{~mm}$ increases the cost of a dipole magnet by $32.6 \%$, and the cost of a quadrupole by $27.7 \%$. The quadrupole costs are further increased by $20 \%$ because of the added focusing needed for the $72^{\circ}$ phase advance per cell.

The tooling costs are estimated to be slightly more than $50 \%$ owing to fixed overhead costs that are independent of the size of the job and to the increase in magnet size. The spool costs are higher than $50 \%$ because of both the increase in aperture and the additional 200 sextupoles needed for operating the machine (see section 4.3.3.3).

The cost of the IR magnets reflects a design with more than half the number of IR magnets in the CDR, having the same focusing properties but lacking a vertical separation scheme. Finally, we assume that the installation and survey costs are half the CDR costs, since there is approximately half the number of elements to install. 


\subsubsection{Other Main Ring Systems}

Since there is only one ring in this design, the quiescent heat load on the cyrogenics system is reduced accordingly. Since the IR's are uniformly distributed in the $\bar{p}$ design, the main ring has eight fold symmetry. We take advantage of this fact by placing the refrigeration/power supply stations at the symmetry points. Thus the number of refrigeration/power supply stations is reduced from 10 in the CDR to eight. In addition, the heat load is reduced from $31.5 \mathrm{KW}$ to $6.95 \mathrm{KW}$. The overall cost in the cryogenics system is $66 \%$ of the COR value.

The cost savings calculation for the vacuum system assumes that both cold and warm vacuum costs are reduced by a factor of two. In addition, we assume that the abort line vacuum and support equipment costs remain fixed, while the insulating vacuum costs are halved. The overall cost of the vacuum system is $64 x$ of the COR cost.

The power supply and quench protection system for the main ring uses half the equivalent number of elements as the CDR, with the exception of components in the IR region and the quench protection micro-processors. Software costs associated with the quench protection system are assumed to be independent of the number of supplies. The cost for the power supply/quench protection system is estimated to be $60 \%$ of the CDR design value.

The number of power supplies for the correction elements necessary for $\overline{p p}$ is reduced by a factor of two relative to the COR with the exception of the additional supplies needed for the 200 extra sextupoles. This results in a $57 \%$ cost relative to the CDR.

Beam utilities such as injection, abort, and RF still need two systems, as in the COR. We assume that the injection costs remain fixed while the RF costs increase by $14 \%$ due to the additional bunch coalescing scheme. The 
abort system cost is now $60 \%$ of the COR figure, reflecting the lower intensities of the two beams.

The costs associated with beam instrumentation, feedback, and control can be classified as either hardware or software related. We assume that all software costs are the same for either $\mathrm{pp}$ or $\overline{\mathrm{pp}}$, while hardware costs scale according to the number of components. The control system for the $\bar{p}$ option has two fewer sector computers and sector microprocessors. The number of tunnel interfaces is halved, but all other components remain the same. The beam instrumentation package contains two-thirds the number of beam loss monitors and half the number of beam position monitors and collimators. The cost of these items is listed in Table 5.4.

Table 5.4

$\overline{\mathrm{p}}$ Collider Component Cost (FY86 k\$)

WBS

$\underline{\text { CDR }}$

$\overline{\mathbf{p}}$

Ratio $\%$

1.2.1.1 Tooling

1.2.1.2 Dipoles

1.2.1.3 Quads

1.2 .7 .4 Spools

1.2.1.5 IR Magnets

1.2.1.6 Installation

1.2 .2 Cryo

1.2 .3 Vacuum

1.2.4 Power Supplies/Quench Prot

1.2.5 Correction Element P.S.

1.2 .6

1.2 .7

1.2 .8

1.2 .9

1.2 .10

1.2 .11

1.2 .12

R.F. System

Feedback Systems

Injection

Abort

Beam Instrumentation

Controls

Safety

2. Conventional Facilities

746

56,031
746,120
39,262
78,108
39,168
42,564
121,137
17,321
26,105
6,942
7,302
4,301
5,201
9,669
12,871
18,016
4,791

576,265

$\begin{array}{rr}31,658 & 56.5 \\ 494,903 & 66.3 \\ 30,086 & 76.6 \\ 57,229 & 73.2 \\ 16,658 & 42.5 \\ 21,282 & 50.0 \\ 79,943 & 66.0 \\ 11,027 & 63.6 \\ 15,107 & 57.8 \\ 3,958 & 57.0 \\ 8,302 & 113.7 \\ 2,253 & 52.4 \\ 5,201 & 100.0 \\ 5,805 & 60.0 \\ 6,882 & 53.5 \\ 15,927 & 88.4 \\ 4,791 & 100.0 \\ & \\ 601,035 & 104.3\end{array}$




\subsection{Conventional Facilities}

The cost of conventional facilities associated with the $\overline{p p}$ option includes additional power for the MEB and holding ring $\$ 2 M$, a change in length of the main ring tunnel and the circumference of MEB $(+\$ 9.71 M)$, elimination of two refrigeration stations $(-\$ 2.4 \mathrm{M})$ and one half of the electronic tunnel enclosures $(-\$ 5 M)$. In the $\overline{p p}$ option, the interaction regions are not clustered, but distributed around the main ring. The non-clustering of the IR's increases the cost of the power distribution system $(+\$ 16 M)$ and increases the cost of bringing "civilization" (e.g. water, security, sanitary facilities) by $\$ 5 M$. The cost of the conventional facilities is $\$ 24.8 \mathrm{M}$ (4\%) more than the figure $(\$ 576.3 M)$ in the CDR. 


\subsection{Conclusions}

It is our conclusion that there would be a net saving in building a single ring $\overline{p p}$ collider. The major cost saving is the need for only a single large collider ring (see Table 5.5). However, there are significant costs associated both with developing the $\bar{p}$ source and with increasing the aperture of the main ring to accommodate antiprotons. We estimate that one would reduce the cost of the SSC by $\$ 242 \mathrm{M}$ by building a $\overline{\mathrm{pp}}$ collider with $\mathscr{L}=10^{32} \mathrm{~cm}^{-2} \mathrm{~s}^{-1}$, instead of the pp collider with $\mathscr{L}=10^{33} \mathrm{~cm}^{-2} \mathrm{~s}^{-1}$ presented in the CDR.

Table 5.5

$\overline{\mathbf{D}}$ Option Total Component Cost (FY86 K\$)

$\begin{array}{lrc} & \overline{\mathbf{p}} & \underline{\text { COR }} \\ \text { Linac } & 25,068 & 25,068 \\ \text { LEB } & 15,585 & 15,585 \\ \text { MEB } & 67,953 & 31,720 \\ \bar{p} \text { Source } & 121,918 & - \\ \text { Holding Ring } & 44,776 & - \\ \text { HEB } & 107,271 & 107,271 \\ \text { Collider } & 1,412,047 & 1,811,174 \\ \text { EDI+Contingency } & 973,687 & 1,019,500 \\ \text { TOTAL } & 2,768,305 & 3,010,318\end{array}$




\section{Chapter 6}

\section{DISCUSSIONS AND CONCLUSIONS}

In this study, we have assessed the antiproton-proton collider option for the SSC. In particular, we have compared a $\overline{p p}$ collider with the pp collider of performance, reliability, cost and physics potential. Our main conclusions are summarized below:

I. A luminosity of $10^{33} \mathrm{~cm}^{-2} \mathrm{~s}^{-1}$ for a $\overline{\mathrm{pp}}$ collider does not appear practical. This is primarily because the bore of the main ring collider magnets must be increased in order to obtain adequate separation of the proton and antiproton beams. In addition, the production of enough antiprotons would require major technical advances. By contrast, the maximum luminosity of the proton proton collider is limited by the number of allowed events per crossing, which could be increased for some experiments.

II. A luminosity of $10^{32} \mathrm{~cm}^{-2} \mathrm{~s}^{-1}$ can reasonably be expected by extrapolating the technology of present CERN and Fermilab sources. Analyzing such a $\overline{p p}$ collider has been the emphasis of our study.

1. We find that for most of the physics objectives, there is simply a loss of a factor of ten in rate compared to the pp collider. Processes involving weak or electromagnetic couplings and/or low mass scales favor the higher luminosity pp option. Event rates for hypothetical processes involving new gauge bosons or composite quarks and leptons coupled strongly to the $\bar{q} q$ annihilation process are roughly equal for the two options. 
2. The best cost estimate of realizing such a collider is that it would cost approximately $\$ 242 M$ less than a pp collider. Although only one ring is required, the added costs both of the larger bore single ring and of the $\bar{p}$ source and holding complex nullify much of the saving.

3. We note that the $\overline{p p}$ collider costs would be somewhat reduced, by about $\$ 45 \mathrm{M}$, if a separate holding ring were not used or if it were demonstrated that a smaller separation of the proton and antiproton beams was sufficient in the main collider ring. However, neither of these possibilities appears justified.

III. Judging from the CERN experience, it appears that $\bar{p} p$ is at least a factor of two worse than pp in reliability of performance.

Finally, we note that we have not considered a low luminosity $\left(\mathscr{L} \simeq 10^{30} \mathrm{~cm}^{-2} \mathrm{~s}^{-1}\right)$ machine without orbit separation. Such a collider would require neither a bright source nor a larger main collider bore, resulting in greater cost savings. Neither have we analyzed the site-specific $\overline{p p}$ option at Fermilab, where savings in both the proton injector and the $\bar{p}$ source might be realized. 